Paralelização de inferência em redes credais utilizando computação distribuída para fatoração de matrizes esparsas

\author{
Ramon Fortes Pereira
}

\author{
DisSERTAÇÃO APRESENTADA \\ $\mathrm{AO}$ \\ Instituto De Matemática e Estatística \\ DA \\ Universidade DE SÃo PAUlo \\ PARA \\ OBTENÇÃO DO TÍTULO \\ $\mathrm{DE}$ \\ Mestre em CiÉnCias
}

Programa: Mestrado em Ciência da Computação

Orientador: Prof. Dr. Júlio Michael Stern - IME-USP 


\section{Paralelização de inferência em redes credais utilizando computação distribuída para fatoração de matrizes esparsas}

Esta versão da dissertação contém as correções e alterações sugeridas pela Comissão Julgadora durante a defesa da versão original do trabalho, realizada em 25/04/2017. Uma cópia da versão original está disponível no

Instituto de Matemática e Estatística da Universidade de São Paulo.

Comissão Julgadora:

- Prof. Dr. Júlio Michael Stern (orientador) - IME-USP

- Prof. Dr. Denis Deratani Mauá - IME-USP

- Prof. Dr. Fábio Gagliardi Cozman - POLI-USP 


\section{Agradecimentos}

Aos Professores Dr. Júlio Michael Stern, Dr. Denis Deratani Mauá, Dr. Fábio Gagliardi Cozman

E à minha família. 


\section{Resumo}

PEREIRA, R. F. Paralelização de inferência em redes credais utilizando computação distribuída para fatoração de matrizes esparsas. 2016. 109 f. Dissertação (Mestrado) - Instituto de Matemática e Estatística, Universidade de São Paulo, São Paulo, 2016.

Este estudo tem como objetivo melhorar o desempenho computacional dos algoritmos de inferência em redes credais, aplicando técnicas de computação paralela e sistemas distribuídos em algoritmos de fatoração de matrizes esparsas. Grosso modo, técnicas de computação paralela são técnicas para transformar um sistema em um sistema com algoritmos que possam ser executados concorrentemente. E a fatoração de matrizes são técnicas da matemática para decompor uma matriz em um produto de duas ou mais matrizes. As matrizes esparsas são matrizes que possuem a maioria de seus valores iguais a zero. E as redes credais são semelhantes as redes bayesianas, que são grafos acíclicos que representam uma probabilidade conjunta através de probabilidades condicionais e suas relações de independência. As redes credais podem ser consideradas como uma extensão das redes bayesianas para lidar com incertezas ou a má qualidade dos dados. Para aplicar a técnica de paralelização de fatoração de matrizes esparsas na inferência de redes credais, a inferência utiliza-se da técnica de eliminação de variáveis onde o grafo acíclico da rede credal é associado a uma matriz esparsa e cada variável eliminada é análoga a eliminação de uma coluna.

Palavras-chave: rede credal, inferência em redes credais, eliminação de variáveis, fatoração de matrizes esparsas. 


\section{Abstract}

PEREIRA, R. F. Parallelization of credal network inference using distributed computing for sparse matrix factorization. 2016. 109 f. Dissertação (Mestrado) - Instituto de Matemática e Estatística, Universidade de São Paulo, São Paulo, 2016.

This study's objective is the computational performance improvement of credal network inference algorithms by applying computational parallel and distributed system techniques of sparse matrix factorization algorithms. Roughly, computational parallel techniques are used to transform systems in systems with algorithms that can be executed concurrently. And the matrix factorization is a group of mathematical techniques to decompose a matrix in a product of two or more matrixes. The sparse matrixes are matrixes which have most of their values equal to zero. And credal networks are similar to Bayesian networks, which are acyclic graphs representing a joint probability through conditional probabilities and their independence relations. Credal networks can be considered as a Bayesian network extension because of their manner of leading to uncertainty and the poor data quality. To apply parallel techniques of sparse matrix factorization in credal network inference the variable elimination method was used, where the credal network acyclic graph is associated to a sparse matrix and every eliminated variable is analogous to an eliminated column.

Keywords: credal network, credal network inference, variables elimination, sparse matrix factorization. 


\section{Sumário}

$\begin{array}{ll}\text { Lista de Tabelas } & \text { ix }\end{array}$

1 Introdução 1

2 Redes Credais e Bayesianas $\quad 3$

2.1 Grafos . . . . . . . . . . . . . . . . . . . . . 3

2.2 Matriz de Adjacência . . . . . . . . . . . . . . . . . . . . . . 3

2.3 Grafos Direcionados . . . . . . . . . . . . . . . . . . . 4

2.4 Grafos Direcionados Acíclicos . . . . . . . . . . . . . . . . . 4

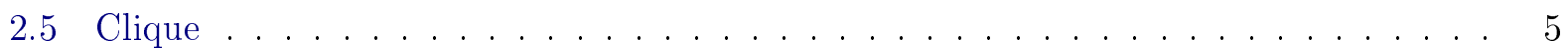

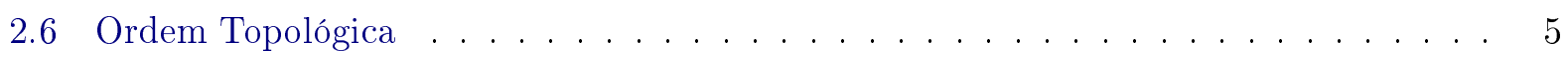

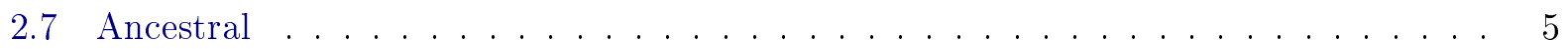

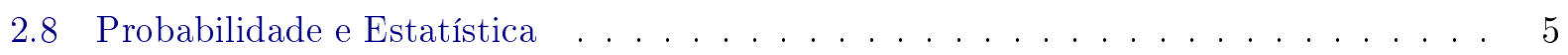

2.9 Variáveis Aleatórias . . . . . . . . . . . . . . . . . . . . 6

2.10 Função de Distribuição . . . . . . . . . . . . . . . . . . . . . . . . . . . . . . . . . .

2.11 Probabilidade Conjunta e Marginal . . . . . . . . . . . . . . . . . . . . 7

2.12 Probabilidade Condicional . . . . . . . . . . . . . . . . . . 8

2.13 Independência . . . . . . . . . . . . . . . . . . . . . . . . . 9

2.14 Teorema de Bayes . . . . . . . . . . . . . . . . . . . . . 10

2.15 Independência Condicional . . . . . . . . . . . . . . . . . . . . . . 11

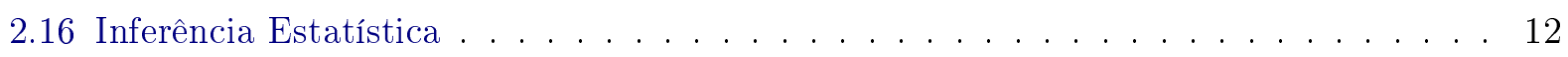

2.17 Redes Bayesianas . . . . . . . . . . . . . . . . . . . . . . 12

2.17 .1 Condição de Markov . . . . . . . . . . . . . . . . . . . 14

2.18 Redes Credais . . . . . . . . . . . . . . . . . . . . . . . . . . . 14

2.19 Potenciais . . . . . . . . . . . . . . . . . . . 17

2.20 Inferência em Redes Bayesianas . . . . . . . . . . . . . . . . . . . . . 17

2.21 Variáveis Requisitadas . . . . . . . . . . . . . . . . . . . . . 19

2.22 Inferência em Redes Credais . . . . . . . . . . . . . . . . . . . . . . . 21

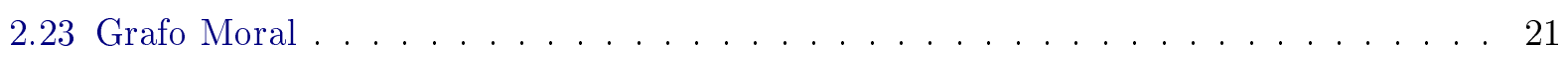

2.24 Grafo Preenchido . . . . . . . . . . . . . . . . . . . . . . . 22

3 Inferência em Redes Credais usando técnicas de Álgebra Linear (Fatoração Simbólica) $\mathbf{2 5}$

3.1 Fatoração de Matrizes aplicada na inferência de redes bayesianas . . . . . . . . . . 25

3.2 Fatoração de Matrizes aplicada na inferência de redes credais . . . . . . . . . . . 25 
3.3 Fatoração Simbólica . . . . . . . . . . . . . . . . . . . 26

3.3 .1 Fatoração de Cholesky . . . . . . . . . . . . . . . . . . . . . . 26

3.3 .2 Ordem de Eliminação . . . . . . . . . . . . . . . . . . . . 27

3.3 .3 Árvore de Dissecção . . . . . . . . . . . . . . . . . . . 27

3.3.4 Heurística de Busca em Largura e a Heurística de Gibbs . . . . . . . . . . . 29

3.3 .5 Árvore de Eliminação . . . . . . . . . . . . . . . . . . . . . 32

3.3.6 Inferências em Rede Credal - Exemplo . . . . . . . . . . . . . . . . . . . 33

3.4 Fatoração Numérica . . . . . . . . . . . . . . . . . . . . 38

3.4.1 Envoltória Convexa ou Fecho Convexo . . . . . . . . . . . . . . 38

3.4 .2 Enumeração de vértices e da faces . . . . . . . . . . . . . . . . . . . . . . . . . . . . . . 39

3.4.3 Programação Linear . . . . . . . . . . . . . . . . . . . . . . . . . 39

3.4.4 Inferências em Rede Credal - Continuação do exemplo . . . . . . . . . . . . . 39

4 Paralelização da inferência em Redes Credais através de computação distribuída $\begin{array}{lr}\text { com OpenMPI } & \mathbf{7 5}\end{array}$

4.1 Computação Paralela . . . . . . . . . . . . . . . . . . . . . . . . . 75

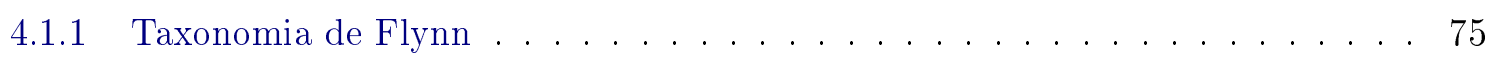

4.1 .2 Computação distribuída - MPI . . . . . . . . . . . . . . . 76

4.2 Biblioteca para Inferência em Redes Credais - Organização do código-fonte . . . . . . 76

4.3 Comparação da inferência linear com a paralela escalável . . . . . . . . . . . . . . . 77

4.3 .1 Tempo de execução . . . . . . . . . . . . . . . . . . . . . . . . . 77

5 Conclusão e trabalhos futuros $\quad \mathbf{7 9}$

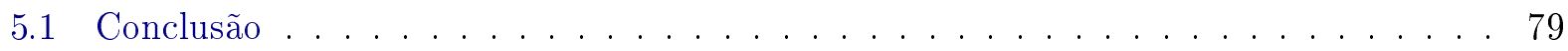

5.2 Trabalhos futuros . . . . . . . . . . . . . . . . . . 79

$\begin{array}{ll}\text { A Rede Credal do exemplo de inferência } & \mathbf{8 1}\end{array}$

A.1 Rede Credal do exemplo A de inferência . . . . . . . . . . . . . . . . . . . 81

A.1.1 Rede Credal com limites inferiores . . . . . . . . . . . . . . . . 81

A.1.2 Rede Credal com limites superiores . . . . . . . . . . . . . . . . 83

A.1.3 Rede Credal Evidência . . . . . . . . . . . . . . . . . . . . . . . . 85

A.1.4 Rede Credal Consulta . . . . . . . . . . . . . . . . . . . . . 86

A.2 Rede Credal do exemplo B de inferência . . . . . . . . . . . . . . . 86

A.2.1 Rede Credal com limites inferiores . . . . . . . . . . . . . 86

A.2.2 Rede Credal com limites superiores . . . . . . . . . . . . . . . . 89

A.2.3 Rede Credal Evidência . . . . . . . . . . . . . . . . . . . . . . . 92

A.2.4 Rede Credal Consulta . . . . . . . . . . . . . . . . . . . . 93

$\begin{array}{ll}\text { Referências Bibliográficas } & 95\end{array}$ 


\section{Lista de Tabelas}

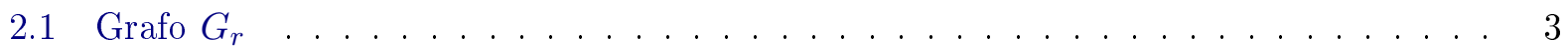

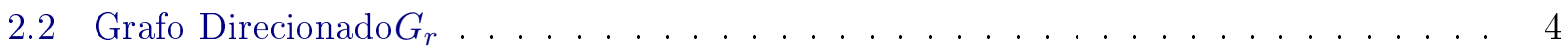

2.3 Exemplo da sala de aula . . . . . . . . . . . . . . . . . . . 8

2.4 Exemplo da sala de aula com probabilidades marginais . . . . . . . . . . . 8

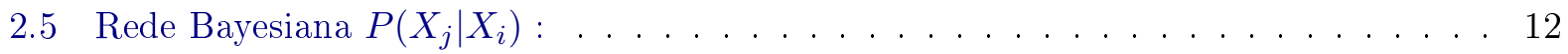

2.6 Rede Credal $P(Y \mid X): \ldots \ldots \ldots \ldots \ldots \ldots \ldots \ldots$

2.7 Eliminação de variáveis: . . . . . . . . . . . . . . . . . . . . . 18

2.8 Variáveis Requisitadas. . . . . . . . . . . . . . . . . . . . . 20

2.9 Grafo Moral. . . . . . . . . . . . . . . . . . . . . . . . . . . 22

2.10 Grafo Preenchido. . . . . . . . . . . . . . . . . . . . 23

3.1 Grafo direcionado - Fatoração Cholesky . . . . . . . . . . . . . 26

3.2 Matriz adjacência - Fatoração Cholesky . . . . . . . . . . . . . . . 26

3.3 Grafo Preenchido. . . . . . . . . . . . . . . . . . . . 27

3.4 Fatoração de Cholesky - Matriz do Grafo Preenchido. . . . . . . . . . . . . . . . . . 27

3.5 Confecção da Árvore de dissecção - Passo $1 \ldots \ldots$. . . . . . . . . . . 28

3.6 Confecção da Árvore de dissecção - Passo $2 \ldots \ldots$. . . . . . . . . . . . . 28

3.7 Confecção da Árvore de dissecção - Passo $3 \ldots \ldots$. . . . . . . . . . . 28

3.8 Grafo e Árvore de dissecção . . . . . . . . . . . . . . . . . . . . . . . . 30

3.9 Árvore BEL-Gibbs . . . . . . . . . . . . . . . . . . . . . . 31

3.10 Árvore de dissecção . . . . . . . . . . . . . . . . . . . . . . . . . . . 31

3.11 Exemplo de Rede Credal do apêndice A . . . . . . . . . . . . . . . . . . . 34

3.12 Grafo Moral da Rede Credal do apêndice A . . . . . . . . . . . . . . . . . . . 36

3.13 Grafo Preenchido da Rede Credal do apêndice A . . . . . . . . . . . . . . . 37

3.14 Árvore de eliminação do exemplo do apêndice A . . . . . . . . . . . . . . . . . 37

4.1 Taxonomia de Flynn . . . . . . . . . . . . . . . . . . . . 76

4.2 Experimento de Inferência em Redes Credais ～. . . . . . . . . . . . . . . 78 


\section{Capítulo 1}

\section{Introdução}

Redes Credais são um tipo dos diversos tipos existentes de redes probabilísticas gráficas que facilitam a representação, especificação e a manipulação de probabilidades conjuntas com uma grande quantidade de variáveis aleatórias. A inferência nestas redes probabilísticas são um mecanismo de aprendizagem e atualização das probabilidades conjuntas através desta aprendizagem.

Conforme Koller e Friedman (2009) e Pourret e Naim (2008), as redes probabilísticas gráficas são aplicadas em diversas áreas como : inteligência artificial, medicina, finanças e bioinformática. E a inferência em redes credais é um problema de complexidade NP-difícil como pode ser visto em Antonucci et al. (2014). NP-difíceis são problemas pelo menos tão difíceis quanto resolver SAT, Cormen et al. (2009). Dado este contexto na análise e síntese de algoritmos, abrem-se os campos de estudo para que estes algoritmos sejam paralelizados de maneira a ganhar aumento de desempenho.

Este trabalho possui um capítulo introdutório dedicado aos conceitos de probabilidade e estatística necessários para o entendimento de uma rede credal e como a inferência estatística trabalha nela. O capítulo seguinte demonstra como a analogia de fatoração de matrizes esparsas pode auxiliar na paralelização do algoritmo de inferência em redes credais. E logo após vem o capítulo que explica a biblioteca implementada durante este trabalho, além de mostrar o resultado do experimento de comparação de inferência em redes credais sem a paralelização contra a inferência paralelizada.

No trabalho da Maranhão (2013), a rede credal é transformada em um conjunto de redes bayesianas. Neste trabalho, a diferença está justamente ao tratar da rede credal como uma rede credal durante toda o processo de inferência. Demonstrando assim, que a fatoração simbólica pode ser aplicada diretamente em uma rede credal. A inferência deste trabalho é aproximada e o algoritmo utilizado é o algoritmo A/R+, da Rocha et al. (2003) e Tessem (1992). 


\section{Capítulo 2}

\section{Redes Credais e Bayesianas}

\section{$2.1 \quad$ Grafos}

Um grafo $G$ é um par de conjuntos $G=(V, E)$, onde o primeiro conjunto (V) é o conjunto de vértices. $\mathrm{E}$ o segundo conjunto $(\mathrm{E})$ é um conjunto de pares ordenados de vértices $(\mathrm{V} \times \mathrm{V})$ indicando quais vértices do conjunto (V) possui conexões entre si, conforme visto em Diestel (2000). Esta conexão é chamada de aresta. Um grafo pode ser representado graficamente como o exemplo abaixo:

Tabela 2.1: Grafo $G_{r}$

Grafo $G_{r}$

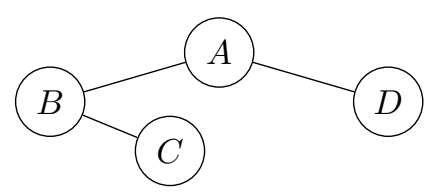

$G_{r}=(V, E)$

$V=\{A, B, C, D\}$

$E=\{(A, D)(A, B),(B, C)\}$

\subsection{Matriz de Adjacência}

Um grafo $\mathrm{G}=(\mathrm{V}, \mathrm{E})$ pode ser representado através de uma matriz booleana. Sendo uma matriz booleana $\mathrm{B}$, onde dada uma linha $i$ e uma coluna $j$, temos $B_{i}^{j}=1$ quando existe a aresta conectando os vértices $i$ e $j$. Caso não exista uma aresta entre os vértices $i$ e $j$ então $B_{i}^{j}=0$. Cada linha da matriz booleana $B$ representa um vértice. $\mathrm{E}$ cada coluna também.

Conforme o exemplo anterior onde o grafo $G_{r}$ foi desenhado, agora vamos representá-lo através de uma matriz booleana $B_{R}$ :

$$
\left(\begin{array}{llllll} 
& & A & B & C & D \\
& & 0 & 1 & 2 & 3 \\
A & 0 & 0 & 1 & 0 & 1 \\
B & 1 & 1 & 0 & 1 & 0 \\
C & 2 & 0 & 1 & 0 & 0 \\
D & 3 & 1 & 0 & 0 & 0
\end{array}\right)
$$

Essa matriz também é chamada de matriz de adjacência do grafo $G_{r}$. 
E é importante observar que toda matriz de adjacência é simétrica Chartrand e Zhang (2012).

\subsection{Grafos Direcionados}

Um grafo direcionado possui dois conjuntos como o grafo. O primeiro conjunto do grafo direcionado é exatamente o mesmo do grafo, que é o conjunto de vértices. O segundo conjunto do grafo direcionado é um conjunto de pares ordenados de vértices indicando quais vértices possuem arcos de ligação, qual o vértice de origem e o vértice de destino. A principal diferença entre o grafo e o grafo direcionado está na mudança do conjunto de arestas para o conjunto de arcos direcionados.

Dado um arco direcionado $(u, v), u$ é dito adjacente para $v$ e $v$ adjacente de $u$.

Um grafo direcionado também pode ser chamado de dígrafo. $\mathrm{E}$ se, para cada par $u, v$ de vértices distintos, no máximo existir um arco direcionado $(u, v)$ ou $(v, u)$, então o dígrafo é um grafo orientado Diestel (2000).

Modificando o exemplo de grafo anterior $G_{r}$ para transformá-lo em um grafo direcionado, temos:

Tabela 2.2: Grafo DirecionadoG $G_{r}$

Grafo Direcionado $G_{r}$

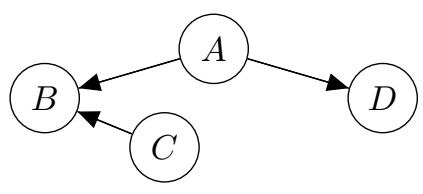

$G_{r}=(V, E)$

$V=\{A, B, C, D\}$

$E=\{(A, D)(A, B),(C, B)\}$

Este exemplo de dígrafo $G_{r}$ também é um grafo orientado.

\subsection{Grafos Direcionados Acíclicos}

Dado um grafo direcionado $\mathrm{G}$ e um conjunto de vértices pertencentes a este grafo $\left\{u_{1}, u_{2}, u_{3}, \ldots, u_{k}\right\}$, onde $u_{i}$ é adjacente para $u_{i+1}$ tal que $\{1<=i<=k-1\}$. Essa sequência de vértices é chamado de passeio ou em inglês de "walk".

$$
w=\left\{u_{1}, \ldots, u_{k}\right\}, w=\left\{u_{1}=u, \ldots, u_{k}=v\right\}
$$

Este passeio também é chamado de passeio $u-v$ no grafo $\mathrm{G}$. A quantidade de arcos direcionados presentes no passeio determinam o tamanho do passeio. Que no caso do passeio w, o tamanho é $k$. Um passeio onde nenhum dos seus arcos direcionados são repetidos é chamado de trajeto ou trajeto direcionado no caso de dígrafos. Um passeio $u-v$ é classificado como aberto quando $u \neq v$ e fechado quando $u=v$.

Um passeio fechado com tamanho mínimo de dois, sem vértices repetidos exceto pelos vértices inicial e final é definido como um ciclo ou ciclo direcionado no caso de dígrafos. 
Um dígrafo sem ciclos é um grafo direcionado acíclico Chartrand e Zhang (2012). O exemplo $G_{r}$ anterior é um grafo direcionado acíclico.

\subsection{Clique}

Um subgrafo $S$ de um grafo $G$ é definido como tendo o conjunto de seus vértices, $V(S)$, sendo um subconjunto do conjunto de vértices de $G, V(G)$. Ou seja, $V(S) \subseteq V(G)$, e também o conjunto de arestas de $S, E(S)$, sendo um subconjunto de conjunto de arestas de $G$. Ou seja, para $S$ ser um subgrafo de $G$, basta que $V(S) \subseteq V(G)$ e $E(S) \subseteq E(G)$.

Um grafo $\mathrm{G}$ é completo quando para todos os seus vértices têm-se dois vértices distintos $v$ e $u$, onde $v$ e $u$ são adjacentes. Um subgrafo $\mathrm{S}$ é completo sob as mesmas condições por ser um grafo também. Um clique em um grafo $G$ é um subgrafo completo em $G$, Bondy e Murty (1982) e Chartrand e Zhang (2012).

\subsection{Ordem Topológica}

Dado um grafo direcionado temos a ordem topológica dos vértices como sendo uma ordem $\left\{x_{1}, x_{2}, x_{3}, \ldots, x_{n}\right\}$ em um grafo com $n$ vértices onde existem arcos direcionados $x_{1} \rightarrow x_{2}, x_{2} \rightarrow x_{3}, \ldots$ e $x_{n-1} \rightarrow x_{n}$.

Logo, se em um grafo direcionado tivermos $x_{i} \rightarrow x_{j}$ então teremos a seguinte ordem topológica: $X_{i}, X_{j}$ onde $i<j$, Koller e Friedman (2009).

\subsection{Ancestral}

Dado um grafo $G=(V, E)$ com os vértices $X$ e $Y, X$ é denominado ancestral de $Y$ se existir um passeio no grafo $\left\{u_{1}, u_{2}, \ldots, u_{k}\right\}$ tal que $u_{1}=x$ e $u_{k}=y$. E da mesma definição, por $X$ ser ancestral de $Y$, então $Y$ é descendente de $X$, Koller e Friedman (2009).

E $X$ é pai de $Y$ se o vértice $X$ é ancestral e adjacente para $Y: X \rightarrow Y$.

\subsection{Probabilidade e Estatística}

Em nosso dia a dia temos que lidar com a incerteza de alguns eventos ou até mesmo a incerteza que temos sobre algum assunto. A probabilidade é uma ferramenta matemática que nos ajuda a lidar com esta incerteza. Um dos primeiros conceitos na probabilidade é o conceito de eventos DeGroot (1989) e Degroot e Schervish (2012).

Supondo que um dado foi lançado. Sabe-se que um dado tem seis lados, logo, os eventos possíveis são que um dos lados saia com a face voltada para cima indicando um dos seis possíveis números $\{1,2,3,4,5,6\}$. Um evento possível é de o dado ter saído com a face de número um $\{1\}$ voltado para cima.

Neste mesmo exemplo, todos os possíveis resultados de lançar-se um dado é que saia uma das faces $\{\{1\},\{2\},\{3\},\{4\},\{5\},\{6\}\}$.

Um evento é um conjunto dos possíveis resultados de um experimento, como o exemplo de lançar-se um dado. Ao invés de modelar-se seis eventos sendo um para cada face do dado, pode-se modelar dois eventos. Onde um evento representa que a face do dado observada possui um número ímpar, e o outro evento representa os números pares. 
Assume-se neste exemplo do experimento de um lançamento de um dado que só sairá um número por vez. Logo, um número da face é um resultado possível, individual e exclusivo deste experimento. O conjunto de todos os resultados possíveis e indivisíveis forma o espaço amostral do experimento $\Omega$ em questão. Para o caso do dado, o espaço amostral $\Omega$ é $\{1,2,3,4,5,6\}$.

O evento é um conjunto formado pelos elementos do espaço amostral. Logo, todo evento $\alpha$ é um subconjunto do espaço amostral.

E para todos os eventos aleatórios oriundos de experimentos aleatórios, a teoria da probabilidade impõe regras. Vamos definir $\Lambda$ como sendo a classe de todos eventos. A teoria da probabilidade exige que:

Considerando que $\emptyset$ é um evento aleatório, então $\emptyset \in \Lambda$. E todo espaço amostral $\Omega$ pertence a $\Lambda, \Omega \in \Lambda$.

Se existir um evento $\alpha$ que pertence à $\Lambda$ e um evento $\beta$ que também pertence à $\Lambda$, então a união deste eventos $\alpha$ e $\beta$ também pertencerá à $\Lambda$.

A classe dos eventos aleatórios deve ser fechada sob o complemento. Assumindo que o complemento de $\alpha$ é $\beta$,onde $\beta=\Omega-\alpha$ e $\alpha \in \Lambda$, então $\beta$ também pertencerá à $\Lambda$.

Com as definições do eventos aleatórios e da sua classe, a probabilidade pode ser definida. A probabilidade pode ser definida matematicamente através da construção axiomática de Kolmogorov (1950):

A probabilidade mapeia os eventos de uma classe $\Lambda$ de eventos de um dado espaço amostral $\Omega$ para um valor real perante as seguintes condições:

1. Se $\alpha$ é um evento que pertence à $\Lambda$, então o valor real é definido através da função $P(\alpha)$, onde $0 \leq P(\alpha) \leq 1$.

2. $P(\Omega)=1$. A probabilidade do espaço amostral é um.

3. Se $\alpha \in \Lambda, \beta \in \Lambda$ e $\alpha \cap \beta=\emptyset$, então $P(\alpha \cup \beta)=P(\alpha)+P(\beta)$.

Mesmo com essa definição única, a probabilidade possui duas grandes interpretações: a subjetiva e a frequentista.

Voltando ao exemplo do experimento do lançamento de um dado, define-se um evento $\alpha$ que representa a saída do número um $\{1\}$.

Do ponto de vista frequentista, a probabilidade do evento $\alpha$ pode ser interpretada como uma proporção do número de ocorrências do evento em relação ao número de repetições do experimento. Assumindo uma probabilidade de $17 \%$ para o evento $\alpha$, este número pode ser interpretado como se ao lançar cem vezes o dado então têm-se uma frequência de dezessete eventos $\alpha$.

Do ponto de vista subjetivo, o mesmo evento $\alpha$ pode ser interpretado como a opinião de alguém sobre a ocorrência deste evento. Como o dado é considerado normal e não viciado, qualquer pessoa pensaria que todos os lados têm a mesma probabilidade de sair. Entretanto, se este dado tiver algum aspecto diferente que afete o resultado do experimento, então a probabilidade irá demonstrar a percepção da pessoa sobre esta alteração, Roussas (1997) e Roussas (2003), Costa (1933).

\subsection{Variáveis Aleatórias}

Para certos experimentos é necessário que os eventos sejam representados através de medidas em números reais, $\mathbb{R}$. Para representar os eventos em números reais usam-se variáveis aleatórias.

Considerando $\beta$ um subconjunto dos números reais $\mathbb{R}$, um evento aleatório $\alpha$ e a classe dos eventos $\Lambda$, têm-se que uma variável aleatória $X$ é uma função real definida no espaço amostral $\Omega,\{X: \Omega \rightarrow \beta \mid \beta \subseteq \mathbb{R}\}$, tal que todo evento $\left\{X^{-1}(x) ; x \in \beta\right\}$ é um evento aleatório $\left\{X^{-1}(x) \in\right.$ $\Lambda\},\left\{X^{-1}(x) \in \Lambda ; \forall x \in \beta\right\}$. Ou dado que $\{\beta \subseteq \mathbb{R}\}$, temos que $\left\{X^{-1}(x) \in \Lambda ; \forall x \in \beta\right\}$ e $\{X: \Omega \rightarrow \beta\}$. Ou $\{X: \Omega \rightarrow \beta \mid \beta \subseteq \mathbb{R}\}$ temos que $X$ é uma variável aleatória se $\left\{X^{-1}(x) \in \Lambda ; \forall x \in \beta\right\}$, James (2013), Roussas (1997) e Roussas (2003). 
As variáveis aleatórias podem ser categorizadas em discretas e contínuas. Pode-se pensar nas variáveis aleatórias discretas como se elas assumissem categorias enumeráveis. E as variáveis aleatórias contínuas assumem valores reais onde podem ter valores infinitos não enumeráveis.

Para referir-se à uma variável aleatória neste texto será usada uma letra maiúscula, como $X$ por exemplo. E para as variáveis discretas, a letra minúscula será utilizada para indicar o valor atribuído a variável aleatória. Como no caso do lançamento de um dado, sendo $\left\{x_{1}=1, x_{2}=2, x_{3}=3, x_{4}=\right.$ $\left.4, x_{5}=5, x_{6}=6\right\}$, teremos que $P\left(X=x_{1}\right)=0,17$ onde $\alpha=\left\{X=x_{1} ; x_{1}=1\right\}$.

\subsection{Função de Distribuição}

Para uma variável aleatória discreta $X$, onde $\beta \subseteq \mathbb{R}$ e $\left\{x_{1}, x_{2}, \ldots, x_{k}\right\} \subset \beta$ temos que $\{X(\alpha) \in$ $\left.\left\{x_{1}, x_{2}, \ldots, x_{k}\right\} ; \forall \alpha \in \Lambda\right\}$ onde $\Lambda \subseteq \Omega$. Então temos a função de probabilidade $p\left(x_{i}\right)=P(X=$ $\left.x_{i}\right), i=1,2, \ldots, k$. Logo, $\sum_{i=1}^{k} P\left(X=x_{i}\right)=1$.

Assumindo uma variável aleatória contínua $X \operatorname{com} \beta=[0,1]$ e $x \in[0,1]$ onde queremos que a probabilidade seja uniformemente distribuída. Existirá o problema que se $x_{1}$ é um ponto em $[0,1]$ então a probabilidade $P\left(X=x_{1}\right)=0$, pelo fato do intervalo $\beta=[0,1]$ ter um número infinito de valores, Roussas (1997), Roussas (2003) e James (2013). Nestes casos, a função de densidade de probabilidade $f(x)$ deve ser usada:

$f(x) \geq 0, \forall x \in \mathbb{R}$ e $\int_{-\infty}^{+\infty} f(x) d x=1, \forall x \in \mathbb{R}$

E para ambos os tipos de variáveis aleatórias temos a função de distribuição de $X$ definida por: $F_{x}(x)=P(X \leq x), x \in \mathbb{R}$.

Esta função de distribuição $F_{x}$ deve satisfazer as seguintes propriedades:

1. $0 \leq F_{x}(x) \leq 1, x \in \mathbb{R}$

2. $F_{x}$ é não decrescente, $x \leq y \Rightarrow F_{x}(x) \leq F_{x}(y)$;

3. $F_{x}$ é contínua à direita;

4. $F_{x}(x) \rightarrow 0$ como $x \rightarrow-\infty$ e $F_{x}(x) \rightarrow 1$ como $x \rightarrow \infty$. Ou $F_{x}(-\infty)=0$ e $F_{x}(\infty)=1$.

Agora com a definição da função de distribuição de variáveis aleatórias pode-se analisar cada caso desta função para as variáveis discretas e contínuas.

Uma função de distribuição para uma variável aleatória discreta $X$ pode ser definida como:

$F_{x}\left(x_{t}\right)=\sum_{i=1}^{t} p\left(x_{i}\right)=P\left(X \leq x_{t}\right)$

E uma função de distribuição para uma variável aleatória contínua é definida:

$F_{x}\left(x_{t}\right)=\int_{-\infty}^{x_{t}} f(x) d x=P\left(X \leq x_{t}\right)$ Ou $\frac{\partial F_{x}}{\mathrm{~d} x}=f_{x}$.

\subsection{Probabilidade Conjunta e Marginal}

Em alguns experimentos aleatórios, o foco dos estudos pode ter mais de uma variável aleatória. Para tratar de um conjunto de variáveis aleatórias é necessário usar o conceito de probabilidade conjunta.

Em uma classe de aula usam-se duas variáveis aleatórias para caracterizarem-se os estudantes: $X$ para a inteligência, que possui o espaço amostral $\Omega$ : \{inteligência alta, inteligência baixa\}; $Y$ para representar as notas de cada aluno com o espaço amostral $\Omega:\{A, B, C, D\}$.

Para este exemplo, a probabilidade conjunta pode ser representada pela tabela abaixo:

E a probabilidade de um evento $\alpha=\{A, A l t a\}$ onde um estudante tenha tirado uma nota $A$ e tenha uma inteligência alta equivale a:

$P(\alpha)=P(\{A$, Alta $\})=0,09$

E no caso das variáveis aleatórias discretas a função de probabilidade pode ser:

$P\left(X=x_{1}, Y=y_{1}\right)=P(X=\{$ Alta $\}, Y=\{A\})=0,09$

Da probabilidade conjunta podemos chegar à probabilidade de cada variável aleatória: 
Tabela 2.3: Exemplo da sala de aula

\begin{tabular}{l|rr|r}
$y \backslash x$ & Alta & Baixa & $\sum X$ \\
\hline$A$ & 0,09 & 0,01 & 0,10 \\
$B$ & 0,16 & 0,04 & 0,20 \\
$C$ & 0,15 & 0,15 & 0,30 \\
$D$ & 0,10 & 0,30 & 0,40 \\
\hline$\sum Y$ & 0,50 & 0,50 & $\sum X, Y=1,00$
\end{tabular}

$P(X)=\sum_{y} P(X, Y)=\sum_{i=1}^{n} P\left(X, Y=y_{i}\right)=\int_{y} f(x, y) d y=\sum_{y} p(X, Y)$

Esta distribuição de probabilidade de uma variável aleatória gerada a partir da distribuição da probabilidade conjunta é chamada de distribuição marginal, James (2013), Roussas (1997), Roussas (2003) e Koller e Friedman (2009). Este nome vem justamente por esta probabilidade ser calculada na margem da tabela de probabilidade conjunta conforme a tabela abaixo:

Tabela 2.4: Exemplo da sala de aula com probabilidades marginais

\begin{tabular}{l|rr|r}
$y \backslash x$ & Alta & Baixa & $P(Y)=\sum_{x} P(X, Y)$ \\
\hline$A$ & 0,09 & 0,01 & 0,10 \\
$B$ & 0,16 & 0,04 & 0,20 \\
$C$ & 0,15 & 0,15 & 0,30 \\
$D$ & 0,10 & 0,30 & 0,40 \\
\hline$P(X)=\sum_{y} P(X, Y)$ & 0,50 & 0,50 & $\sum P(X, Y)=1,00$
\end{tabular}

\subsection{Probabilidade Condicional}

No exemplo anterior, os estudantes foram caracterizados pela inteligência e pelas notas obtidas. Estes dois eventos estão relacionados, tanto da inteligência quanto da nota, de maneira que pode-se aprender sobre um evento quando conhece-se o outro. A probabilidade condicional é um mecanismo para atualizar a probabilidade de um evento relacionado a outro, quando este segundo evento ocorreu.

Sendo $\alpha$ um evento que identifica a inteligência de um estudante, a probabilidade deste evento $\alpha$ será alterada pelo fato da nota obtida ser $\{A\}$. A nota obtida é representada como o evento $\beta$. Pode-se representar esta situação através da probabilidade condicional:

$$
\begin{aligned}
& P(\alpha \mid \beta)=\frac{P(\alpha \cap \beta)}{P(\beta)} \\
& P(\alpha \mid \beta=\{A\})=\frac{P(\alpha \cap \beta=\{A\})}{P(\beta=\{A\})}
\end{aligned}
$$

Considerando a tabela anterior calcula-se a probabilidade do estudante de ser inteligente dado que ele tirou nota $A$ :

$$
P(\alpha=\{\text { Alta }\} \mid \beta=\{A\})=\frac{P(\alpha=\{\text { Alta }\} \cap \beta=\{A\})}{P(\beta=\{A\})}=\frac{P(\alpha=\{\text { Alta }\}, \beta=\{A\})}{P(\beta=\{A\})}=\frac{0,09}{0,10}=0,009
$$

Uma outra visão da probabilidade condicional e o seu mecanismo de atualização da probabilidade através da ocorrência de eventos é utilizando o diagrama de Venn. Neste diagrama, dentro do plano que representa o espaço amostral $\Omega$ colocam-se os dois eventos:

$$
P(\alpha \mid \beta)=\frac{P(\alpha \cap \beta)}{P(\beta)}
$$




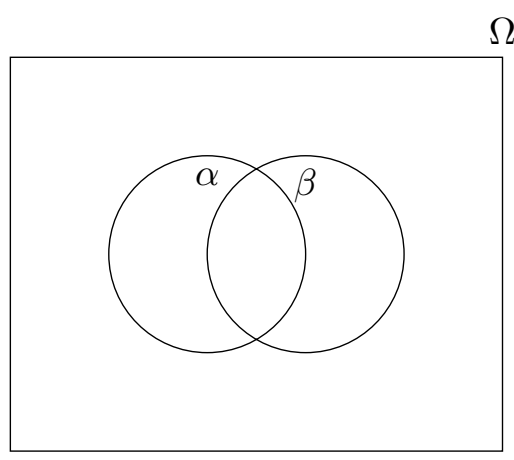

E na ocorrência do evento $\beta$ : $P(\alpha \mid \beta)=\frac{P(\alpha \cap \beta)}{P(\beta)}$
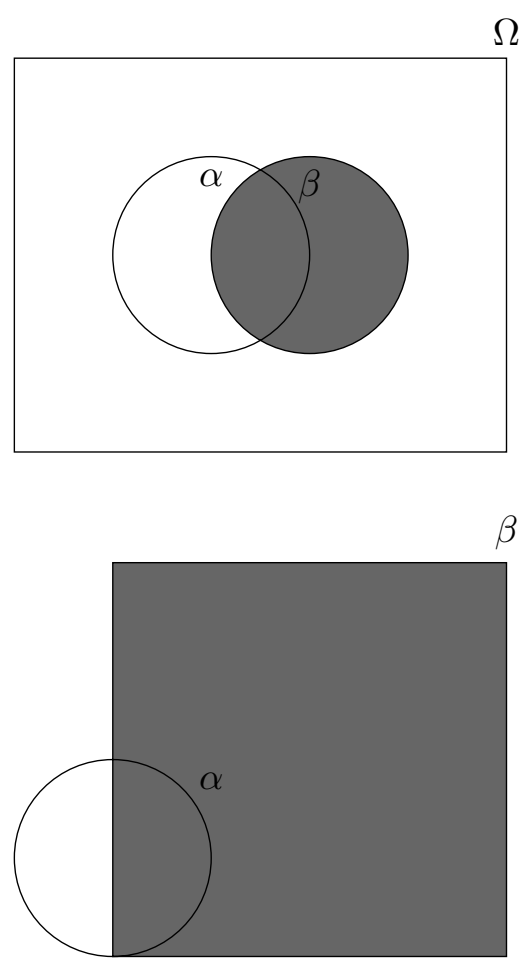

A probabilidade condicional representa esta nova probabilidade após o evento $\beta$ ocorrer: $P(\alpha \mid \beta)=\frac{P(\alpha, \beta)}{P(\beta)}$

E os diagramas mostram a área do círculo $\alpha$ como sendo a probabilidade do evento $\alpha$. Esta área $\alpha$ mostra a probabilidade condicional de $\alpha$ dado que $\beta$ aconteceu através da transição dos diagramas, Roussas (1997), Roussas (2003) e James (2013).

\subsection{Independência}

Para alguns experimentos aleatórios, os eventos escolhidos podem ser independentes. Eventos independentes significa que um evento não altera a probabilidade da ocorrência do outro evento, Roussas (1997) e Roussas (2003).

Esta independência é definida como:

$P(\alpha \mid \beta)=P(\alpha)$

Dados os eventos independentes $\alpha, \beta$, têm-se que a probabilidade do evento $\alpha$ não é modificada na ocorrência do evento $\beta$. E a independência também pode ser expressa por: 
$P(\alpha \cap \beta)=P(\alpha, \beta)=P(\alpha) \cdot P(\beta)$

Um exemplo seria o cálculo da probabilidade de um lançamento de dois dados em instantes diferentes e lugares diferentes. O evento em questão seria que os números sejam um $\{1\}$ em ambas as faces dos dados.

Como estes eventos são independentes então teríamos:

$P(\{$ Face do dado ser 1$\})=\frac{1}{6}$

$P(\{$ Faces dos dados serem 1 em ambos $\})=P(\{$ Face do dado ser 1$\}) \cdot P(\{$ Face do dado ser 1\}) $=\frac{1}{6} \cdot \frac{1}{6}=\frac{1}{36}$.

\subsection{Teorema de Bayes}

Pela definição de probabilidade condicional temos:

$$
P(\alpha \mid \beta)=\frac{P(\alpha \cap \beta)}{P(\beta)} \Leftrightarrow P(\alpha \cap \beta)=P(\alpha \mid \beta) . P(\beta)
$$

E dado que temos eventos mutuamente exclusivos e exaustivos $A_{1}, A_{2}, \ldots$ Ou seja, $A_{i} \cap A_{j}=\emptyset$ para todo $i \neq j$ e $\cup A_{i}=\Omega$. Então os eventos $A_{i}$ formam uma partição do espaço amostral como a figura abaixo mostra:

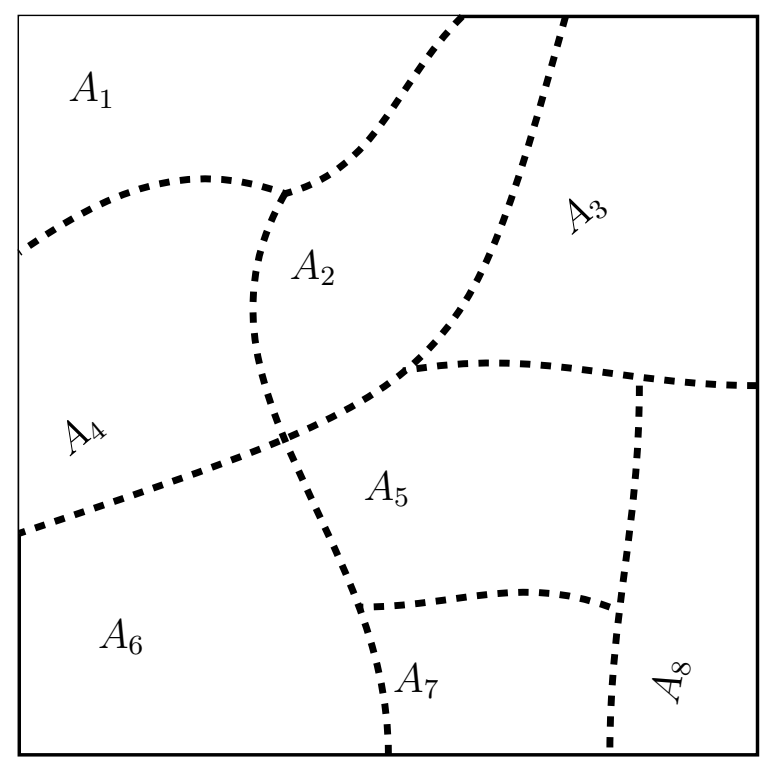

$\Omega$

Dentro deste mesmo espaço amostral, vamos supor a existência de um evento $\beta$ : 


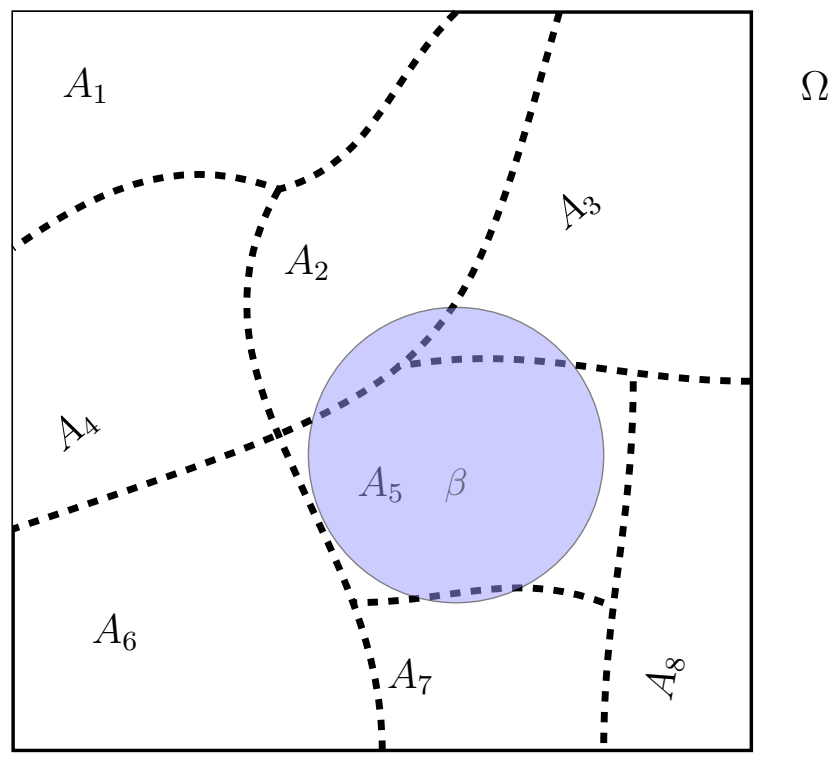

Pelo teorema da probabilidade total pode-se escrever a probabilidade do evento $\beta$ através das probabilidades condicionais de $\beta$ em relação a partição $A_{i}$ :

$$
P(\beta)=\sum_{i} P\left(A_{i}\right) \cdot P\left(\beta \mid A_{i}\right), \forall \beta \in \Lambda .
$$

E com a fórmula de Bayes temos as probabilidades condicionais dos eventos da partição dado o evento $\beta$ :

$$
P\left(A_{i} \mid \beta\right)=\frac{P\left(A_{i} \cap \beta\right)}{P(\beta)}=\frac{P\left(A_{i}\right) \cdot P\left(\beta \mid A_{i}\right)}{\sum_{j} P\left(A_{j}\right) \cdot P\left(\beta \mid A_{j}\right)} .
$$

A fórmula de Bayes é importante por nos formalizar uma maneira de aprender sobre a probabilidade condicional $P(\alpha \mid \beta)$ através do inverso $P(\beta \mid \alpha)$ e sem a necessidade de conhecermos a probabilidade do evento $\beta, P(\beta)$, Koller e Friedman (2009), Roussas (1997), Roussas (2003) e James (2013).

\subsection{Independência Condicional}

Independência condicional é a independência de duas ou mais variáveis aleatórias condicionadas à uma outra terceira variável aleatória, Koller e Friedman (2009).

Considere duas variáveis aleatórias $B, Y$ que fazem parte da probabilidade condicional de uma variável $A: P(A \mid B, Y)$. A variável $A$ é independente condicionalmente de $Y$ dado que a informação da variável $B$ já é suficiente para determinar a probabilidade condicional de $A$. Ou seja, $A$ é independente de $Y(A \perp Y)$. Então, usa-se o símbolo $\perp$ para representar a independência, considerando que temos informações da variável $B: P(A \perp Y \mid B)$. Logo, isso implica que se $P(A \perp Y \mid B)$ então:

$P(A \mid Y, B)=P(A \mid B)$ ou que $P(Y, B)=0$.

Desta definição da probabilidade condicional pode-se obter também que, dado $P(A \perp Y \mid B)$ :

$$
P(A, Y \mid B)=P(A \mid B) \cdot P(A \mid Y) .
$$




\subsection{Inferência Estatística}

Inferência estatística pode ser vista como uma função do espaço amostral para um espaço euclidiano. E mais informalmente, a estatística pode ser definida como a função que consegue ser calculada na observação de um evento aleatório Bickel e Doksum (2001).

A inferência estatística é um método para aprender mais sobre uma determinada variável aleatória em uma dada ditribuição de probabilidade.

A função de distribuição de uma variável aleatória de um experimento aleatório pode ser parametrizável. A maioria das funções de distribuição mais famosas são patrametrizadas, como no caso da função de distribuição normal que recebe os parâmetros média $(\mu)$ e desvio padrão $(\sigma)$.

Por sua vez, estes parâmetros também podem ser considerados variáveis aleatórias, que possuem suas próprias funções de distribuição. A inferência estatística possui ferramentas para auxiliar os usuários de probabilidade na busca pelos valores destes parâmetros. Desta maneira, a função de distribuição do experimento aleatório representará as variáveis de maneira mais verdadeira possível ou o mais próximo da realidade.

A inferência bayesiana é uma inferência estatística. Na inferência bayesiana utiliza-se dados coletados do experimento para aprender mais sobre os parâmetros da função de distribuição das variáveis aleatórias. Estes dados coletados são as estatísticas do experimento aleatório.

Como o parâmetro é uma variável aleatória, a função de distribuição do parâmetro antes de obterem-se as estatísticas é chamada de função de distribuição à priori.

E após a obtenção de estatísticas da amostra do experimento aleatório, utilizam-se os procedimentos formais da inferência bayesiana para atualizar a função de distribuição dos parâmetros. Essa nova ou mais atualizada função de distribuição dos parâmetros é chamada de função de distribuição posterior.

Neste contexto da inferência bayesiana, o objetivo é que o valor do parâmetro seja obtido ou que tenha um melhor valor aproximado possível. Para este valor é que calcula-se o estimador bayesiano. Sendo o estimador bayesiano a melhor estimativa para o parâmetro, na inferência bayesiana, Roussas (1997), Roussas (2003) e Degroot e Schervish (2012).

\subsection{Redes Bayesianas}

Uma rede bayesiana é uma representação de uma distribuição de probabilidade conjunta através de um grafo acíclico direcionado. Cada vértice do grafo representa uma variável aleatória Pearl (1988). E cada aresta representa a probabilidade condicional das variáveis envolvidas nos vértices. Se $X_{i} \rightarrow X_{j}$ ou $X_{i}$ é adjacente para $X_{j}$ em uma ordem topológica $\left\{X_{i}, X_{j}\right\}$, então esses dois vértices representam a probabilidade condicional de $X_{j}$ dado $X_{i}, P\left(X_{j} \mid X_{i}\right)$.

$$
P\left(X_{j} \mid X_{i}\right) \text { : }
$$

Tabela 2.5: Rede Bayesiana $P\left(X_{j} \mid X_{i}\right)$ :

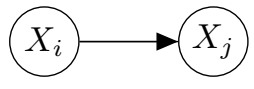

A rede bayesiana é a representação de uma probabilidade conjunta na forma das probabilidades condicionais exitentes na distribuição, deixando esta representação explícita através de um grafo direcionado acíclico. Sendo assim, para cada vértice ou variável aleatória $X_{i}$ representam-se as independências condicionais do modelo por : 
$X_{i}:\left(X_{i} \perp\right.$ Não Descendentes de $X_{i} \mid$ Pais de $X_{i}$ em $\left.G\right)$

Toda variável aleatória $X_{i}$ é independente condicionalmente das variáveis não descendentes de $X_{i}$ dados os pais desta variável aleatória $X_{i}$.

A rede bayesiana pode ser definida como um conjunto $B$ de par ordenado $B:(G, P)$. Onde $G$ é um grafo, $G=(V, E)$, conforme definido anteriormente. E cujos vértices são variáveis aleatórias e as suas arestas representam os pais de cada variável aleatória. E os vértices e as arestas também representam as relações de independência condicional. E $P$ representa a probabilidade conjunta das variáveis aleatórias que formam os vértices do grafo $G$. Essa probabilidade $P$ pode ser representada pelas probabilidades condicionais de cada variável aleatória, através do produtório das mesmas:

$P\left(X_{1}, \ldots, X_{n}\right)=\prod_{i=1}^{n} P\left(X_{i} \mid\right.$ Pais de $X_{i}$ em $\left.G\right)$

Pelo teorema da multiplicação ou teorema da probabilidade, James (2013), composta temos que:

1. $P(A \cap B)=P(A, B)=P(A) \cdot P(B \mid A)=P(B) \cdot P(A \mid B)$

2. $P\left(A_{1} \cap A_{2} \cap \ldots \cap A_{n}\right)=P\left(A_{1}, A_{2}, \ldots, A_{n}\right)$

$=P\left(A_{1}\right) \cdot P\left(A_{2} \mid A_{1}\right) \cdot P\left(A_{3} \mid A_{1} \cap A_{2}\right) \ldots P\left(A_{n} \mid A_{1} \cap \ldots \cap A_{n-1}\right)$

$=P\left(A_{1}\right) \cdot P\left(A_{2} \mid A_{1}\right) \cdot P\left(A_{3} \mid A_{1}, A_{2}\right) \ldots P\left(A_{n} \mid A_{1}, \ldots, A_{n-1}\right)$

E considerando o teorema da multiplicação, a probabilidade conjunta $P$ pode ser reescrita :

$P\left(X_{1}, X_{2}, \ldots, X_{n}\right)=\prod_{i=1}^{n} P\left(X_{i} \mid X_{1}, \ldots, X_{i-1}\right)$

E considerando que o grafo $G$ da rede bayesiana representa as independências condicionais da probabilidade $P$ para qualquer variável aleatória $X_{i}$, pertencente a rede bayesiana :

$X_{i}:\left(X_{i} \perp\right.$ Não Descendentes de $X_{i} \mid$ Pais de $X_{i}$ em $\left.G\right)$

E tendo que quando $(A \perp Y \mid B)$ então $P(A \mid B, Y)$ é igual a $P(A \mid B)$. A probabilidade conjunta $P$ pode ser reescrita :

$$
\begin{aligned}
& P\left(X_{1}, X_{2}, \ldots, X_{n}\right)=\prod_{i=1}^{n} P\left(X_{i} \mid X_{1}, \ldots, X_{i-1}\right) \\
& =\prod_{i=1}^{n} P\left(X_{i} \mid \text { Pais de } X_{i} \text { em } G, Z\right)
\end{aligned}
$$

Onde $Z$ é o conjunto de variáveis aleatórias ou vértices não descendentes de $X_{i}$ tal que :

$$
\left(X_{i} \perp Z \mid \text { Pais de } X_{i} \text { em } G\right)
$$

Então temos que :

$$
P\left(X_{1}, X_{2}, \ldots, X_{n}\right)=\prod_{i=1}^{n} P\left(X_{i} \mid \text { Pais de } X_{i} \text { em } G\right)
$$

Logo, uma rede bayesiana $B=(G, P)$ é composta por uma probabilidade conjunta $P$ e um grafo acíclico direcionado $G$. E $G$ demonstra as independências condicionais exitentes em $P$, levando à correta fatoração :

$$
P\left(X_{1}, X_{2}, \ldots, X_{n}\right)=\prod_{i=1}^{n} P\left(X_{i} \mid \text { Pais de } X_{i} \text { em } G\right)
$$

Considerando somente as variáveis necessárias para representar a probabilidade conjunta em um produtório de probabilidades condicionais, Colla (2007) e Koller e Friedman (2009). 


\subsubsection{Condição de Markov}

A Rede Bayesiana demonstra de maneira clara a relação de dependência e independência das variáveis aleatórias, pois cada variável é condicionalmente independente de seus não-descendentes não-pais dados seus pais. Esta é a Condição de Markov. Desta condição surge uma única distribuição conjunta de probabilidades Bondy e Murty (1982):

$$
P(X)=\prod_{i=1}^{n} P\left(X_{i} \mid \text { pais }\left(X_{i}\right)\right)
$$

Onde pais $\left(X_{i}\right)$ são vértices pais de $X_{i}$.

\subsection{Redes Credais}

Rede Credal é uma rede probabilística gráfica que representa uma probabilidade conjunta e suas relações de independência através de um grafo. Sendo assim, semelhante à rede bayesiana com a diferença de que ao invés de representar probabilidades igual a rede bayesiana faz, a rede credal representa probabilidades imprecisas Cozman (2000), Antonucci et al. (2014).

Na rede bayesiana, cada variável possui a sua função de distribuição. Já na rede credal, cada variável possui um conjunto fechado convexo de medidas de probabilidades Cozman (2000), Maranhão (2013). Permitindo que uma variável possa estar associada a uma inequação probabilística.

Conforme Maranhão (2013) enuncia "Um conjunto convexo de medidas de probabilidades $K$ é tal que se $P_{1}$ e $P_{2}$ pertencem a ele, então $\alpha \cdot P_{1}+(1-\alpha) \cdot P_{2}$ também pertencerá, para $\alpha \in[0,1]$ ".

Um exemplo de rede credal seria:

Tabela 2.6: Rede Credal $P(Y \mid X)$ :

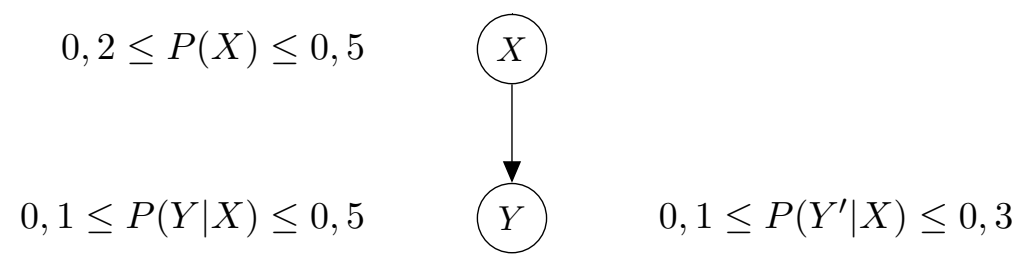

Onde $X$ e $Y$ são variáveis aleatórias e existe a probabilidade condicional $P(Y \mid X)$. E a probabilidade de $X$ é definida através de um conjunto credal, assim como a probabilidade condicional de $Y \mid X$.

Como as variáveis aleatórias podem ter as suas probabilidades representadas por conjuntos credais, a rede credal pode ser vista como uma generalização de uma rede bayesiana. Ou a rede bayesiana pode ser vista como um caso específico de uma rede credal.

\section{Independência}

Dentro das redes credais existem os conceitos chamados extensões que podem ser vistas como categorias de redes credais. Onde cada categoria possui algumas propriedades que são atendidas pelos conjuntos fechados convexos de medidas de probabilidades. Neste estudo a extensão de rede credal utilizada é a extensão forte. A extensão forte significa que a rede credal atende a propriedade de independência forte.

Dado duas variáveis aleatórias $X$ e $Y$ com seus conjuntos fechados convexos de medidas de probabilidades $K(X, Y)$, elas são independentes se os pontos extremos de seus conjuntos credais 
satisfazem a independência estocástica. Ou seja, para cada ponto extremo de $x \in X$ e $y \in Y$ têm-se $\forall p(x \mid y) \in \operatorname{ext}[K(X \mid Y)] \mid p(x \mid y)=p(x)$ e $\forall p(y \mid x) \in \operatorname{ext}[K(Y \mid X)] \mid p(y \mid x)=p(y)$, Cozman (2000), Maranhão (2013) e Antonucci et al. (2014).

Um ponto extremo de um conjunto credal $K$ é um elemento pertencente ao conjunto credal $K$ que não pode ser representado como uma combinação convexa de outros elementos deste conjunto credal $K$ Antonucci et al. (2014). A notação utilizada para representar os pontos extremos de um conjunto credal $K$ será ext $[K(X)]$ semelhante a utilizada em Antonucci et al. (2014).

\section{Marginalização e Probabilidade Condicional}

Dada uma probabilidade conjunta $P(X, Y)$ representada por um conjunto $K(X, Y)$ credal, o conjunto credal $K^{\prime}(X)$ que representa a conjunto credal da probabilidade marginal de $X$, pode ser encontrada através da equação abaixo:

$K^{\prime}(X)=\sum_{Y}^{\otimes} K(X, Y)=C H\left\{P(X) \mid P(x)=\sum_{y \in Y} P(x, y), \forall x \in X, \forall(P(x, y)) \in \operatorname{ext}[K(X, Y)]\right\}$

Onde $C H$ representa a operação de fecho convexo Cormen et al. (2009) e Antonucci et al. (2014), Avis e Fukuda. (1992). Isto quer dizer que para marginalizar o conjunto credal $K(X, Y)$, basta marginalizar os pontos extremos deste conjunto credal e fazer a operação de fecho convexo nos pontos extremos marginalizados.

Este processo é similar para a probabilidade condicional de $P(X \mid Y)$ com o conjunto credal $L(X \mid Y)$ conforme equação abaixo, assumindo $P(y)>0 \mid \forall(P(y)) \in L(Y)$ Antonucci et al. (2014):

$L(X \mid Y)=C H\left\{P(X \mid Y) \mid P(x \mid y)=\frac{P(x, y)}{\sum_{x \in X} P(x, y)}, \forall x \in X, \forall y \in Y, \forall(P(x, y)) \in \operatorname{ext}[L(X, Y)]\right\}$

Onde para se obter a probabilidade condicional $P(X \mid Y)$ e seu conjunto credal condicional $L(X \mid Y)$, basta dividir a probabilidade conjunta $P(x, y)$ pela probabilidade marginal de $P(y)$ de todos os pontos extremos e depois realizar a operação de fecho convexo conforme explicado em Antonucci et al. (2014) e Cormen et al. (2009), Avis e Fukuda. (1992).

E com estas definições um conjunto credal de probabilidades conjuntas pode ser definido através da equação abaixo:

$K^{\prime}(X, Y)=C H\{P(X, Y) \mid P(x, y)=P(x \mid y) P(y), \forall x \in X, \forall y \in Y, \forall(P(y)) \in \operatorname{ext}[K(Y)], \forall(P(x \mid y)) \in$ $\operatorname{ext}[K(X \mid Y)]\}$

Com os pontos extremos das probabilidades marginal e condicional chega-se aos pontos extremos da probabilidade conjunta. E estes pontos extremos são aplicados ao algoritmo para achar o fecho convexo, Antonucci et al. (2014). Este fecho convexo é suficiente para chegar ao conjunto credal da probabilidade conjunta.

\section{Condição de Markov}

Assim como na rede bayesiana, uma rede credal tem suas independências codificadas através de um grafo direcionado acíclico.

Essas independências codificadas é justamente a condição de Markov que define para uma variável $X_{i}$

$\left(X_{i} \perp Z \mid\right.$ Pais de $X_{i}$ em $\left.G\right)$

Onde $G$ é o grafo direcionado acíclico que representa a rede credal. E $Z$ é o conjunto de variáveis não descendentes de $X_{i}$. $X_{i}$ é independente de $Z$ dados os pais de $X_{i}$ Antonucci et al. (2014)

Com essa condição de markov obtida nas redes probabilísticas gráficas como as redes bayesianas e credais, a especificação dos estados de uma probabilidade conjunta fica mais simplificada e menos trabalhosa. Assim como os intervalos probabilísticos das redes credais expressam melhor a realidade da imprecisão da informação.

Logo a probabilidade conjunta de uma rede credal pode ser definida pela expressão:

$P\left(X_{1}, X_{2}, \ldots, X_{n}\right)=\otimes_{i=1}^{n} P\left(X_{i} \mid\right.$ Pais de $X_{i}$ em $\left.G\right)$

$K\left(X_{1}, X_{2}, \ldots, X_{n}\right)=\otimes_{i=1}^{n} K\left(X_{i} \mid\right.$ Pais de $X_{i}$ em $\left.G\right)$

Onde $G$ é o grafo acíclico direcionado e $X_{i}$ é o vértice e variável aleatória pertencente ao $G$ e a probabilidade conjunta. E o operador $\otimes$ é chamado de composição sendo definido como: 
$K(X \mid Y) \otimes K(Y)=C H\{P(x \mid y) \cdot P(y), \forall x \in X, \forall y \in Y, \forall(P(y)) \in \operatorname{ext}[K(Y)], \forall(P(x \mid y)) \in$ $\operatorname{ext}[K(X \mid Y)]\}$

Significando que a multiplicação dos pontos extremos das probabilidades marginais e condicionais em questão são utilizados para depois achar o fecho convexo.

\section{Regra de Bayes}

Conforme visto anteriormente a definição da fórmula de Bayes, onde existe o seguinte cenário:

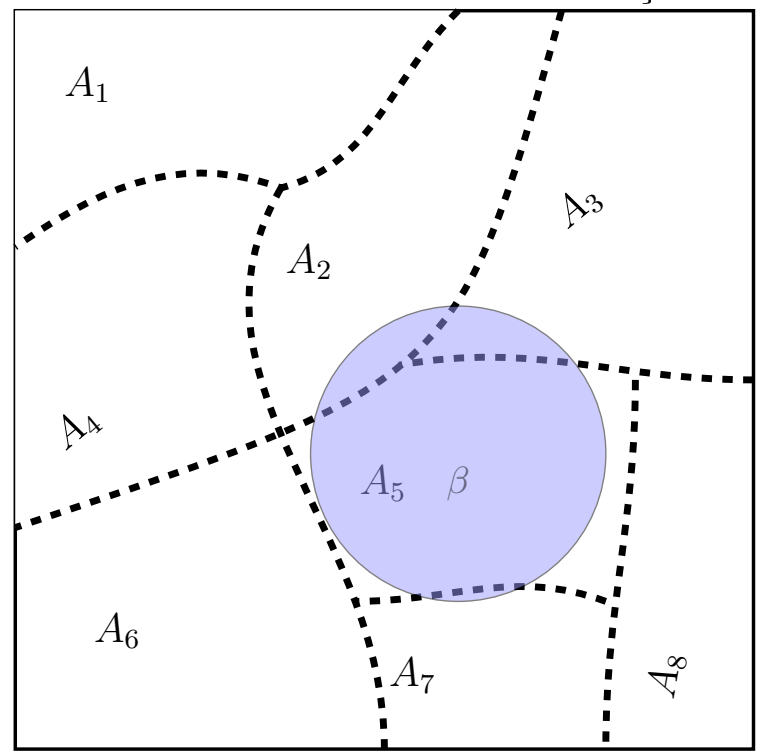

$P(\alpha \mid \beta)=\frac{P(\alpha \cap \beta)}{P(\beta)} \Rightarrow P(\alpha \cap \beta)=P(\alpha \mid \beta) \cdot P(\beta)$

E como $\sum_{i} P\left(A_{i}\right)=1$, sendo $A_{i}$ as partições de $\Omega$, temos pelo teorema da probabilidade total Roussas (1997), James (2013) e Roussas (2003) que

$P(\beta)=\sum_{i} P\left(\beta \cap A_{i}\right)=\sum_{i} P\left(A_{i}\right) P\left(\beta \mid A_{i}\right)$

E através da fórmula de Bayes, pode-se chegar a probabilidade atualizada das partições:

$P\left(\alpha_{i} \mid \beta\right)=\frac{P\left(A_{i} \cap B\right)}{P(B)}=\frac{P\left(A_{i}\right) \cdot P\left(B \mid A_{i}\right)}{\sum_{j} P\left(A_{j}\right) \cdot P\left(B \mid A_{j}\right)}$

E com o operador composição $\otimes$ das redes credais, têm-se:

$K(A \mid B)=C H\left\{P(a \mid b)=\frac{P(a \cap b)}{P(b)}, \forall a \in A, \forall b \in B, \forall(P(a \cap b)) \in \operatorname{ext}[K(A \cap B)], \forall(P(b)) \in\right.$ $\operatorname{ext}[K(B)]\}$

Então:

$K(A, B)=K(A \cap B)=K(A \mid B) \otimes K(B)$

$\mathrm{E}$

$K_{0}(A)=C H\left\{P(x) \geq 0, \forall x \in A, \sum_{x \in A} P(x)=1\right\}$

Define-se a marginalização para um conjunto credal basedo em seus pontos extremos:

$\sum_{\mathrm{E}}^{\otimes} K\left(A_{i}\right)=C H\left\{\sum_{i} P\left(\alpha_{i}\right), \forall \alpha_{i} \in A_{i}, \forall\left(P\left(\alpha_{i}\right)\right) \in \operatorname{ext}\left[K\left(A_{i}\right)\right]\right\}$

$K(B)=\sum_{i}^{\otimes} K\left(B \cap A_{i}\right) \Rightarrow K(B)=\sum_{i}^{\otimes} K(A) \otimes K\left(B \mid A_{i}\right)$

$\mathrm{E}$ com a fórmula de Bayes:

$K\left(A_{i} \mid B\right)=C H\left\{\frac{P\left(\alpha_{i} \cap \beta\right)}{P(\beta)}, \forall \alpha_{i} \in A_{i}, \forall \beta \in B, \forall\left(P\left(\alpha_{i} \cap \beta\right)\right) \in \operatorname{ext}\left[K\left(A_{i} \cap B\right)\right], \forall(P(\beta)) \in \operatorname{ext}[K(B)]\right\}$

E definindo um novo operador $\frac{K(A)}{K(B)}$

$\frac{K(A \cap B)}{K(B)}=C H\{P(\alpha \cap \beta), \forall \alpha \in A, \forall \beta \in B, \forall(P(\alpha \cap \beta)) \in \operatorname{ext}[K(A \cap B)], \forall(P(\beta)) \in \operatorname{ext}[K(B)]\}$

$\mathrm{E}$ conforme Antonucci et al. (2014), têm-se a regra de Bayes para o conjunto credal:

$K\left(A_{i} \mid B\right)=\frac{K\left(A_{i} \cap B\right)}{K(B)}=\frac{K\left(A_{i}\right) \otimes K\left(B \mid A_{i}\right)}{\sum_{j}^{\otimes} K\left(A_{j}\right) \otimes K\left(B \mid A_{j}\right)}$ 


\subsection{Potenciais}

Define-se potencial $\phi$ como sendo uma tabela de números reais para um conjunto finito de variáveis. Este conjunto de variáveis é denominado domínio, cuja notação será $\operatorname{dom}(\phi)$. Como a tabela de distribuição de probabilidades é um conjunto de números reais associado às variáveis aleatórias, ela é um caso especial de potencial, Colla (2007).

Os potenciais podem ser marginalizados ou multiplicados entre si. A multiplicação de pontenciais tem as seguintes propriedades:

- P.1 $\operatorname{dom}\left(\phi_{1} \cdot \phi_{2}\right)=\operatorname{dom}\left(\phi_{1}\right) \cup \operatorname{dom}\left(\phi_{2}\right)$

- P.2 Propriedade comutativa: $\phi_{1} . \phi_{2}=\phi_{2} . \phi_{1}$

- P.3 Propriedade associativa: $\left(\phi_{1} . \phi_{2}\right) \cdot \phi_{3}=\phi_{1} \cdot\left(\phi_{2} \cdot \phi_{3}\right)$

- P.4 Existência da Unidade:o número 1 é o potencial de um domínio vazio e $1 . \phi=\phi$

\subsection{Inferência em Redes Bayesianas}

Inferência em Redes Bayesianas significa que dada uma rede bayesiana e um evento ocorrido, e se as probabilidades são afetadas por este evento, então as probabilidades são recalculadas de maneira que se entenda melhor a variável aleatória que é focada como objeto de estudo.

\section{Método de Eliminação de variáveis}

A inferência em uma rede bayesiana consitui-se de primeiro ter uma probabilidade conjunta representada pela rede bayesiana:

$P\left(X_{1}, X_{2}, \ldots, X_{n}\right)=\prod_{i} P\left(X_{i} \mid\right.$ Pais de $X_{i}$ em $\left.G\right)$

Onde as probabilidades condicionais ou os pais dos vértices são representados pelo grafo acíclico direcionado.

Quando ocorre um evento relacionado a um vértice e variável aleatória, no caso $X_{e}$, têm-se variáveis que estão na distribuição conjunta e são afetadas. Essas variáveis afetadas tem as suas probabilidades alteradas.

As variáveis de uma rede bayesiana que questionamos para saber se a probabilidade foi afetada por $X_{e}$, pode ser definida como $X_{q}$.

$X_{e}$ e $X_{q}$ podem ser somente uma variável aleatória cada ou podem representar um conjunto de variáveis aleatórias.

Esta abordagem de questionarmos um conjunto de variáveis $X_{q}$ dado a ocorrência de eventos pertinentes à variáveis $X_{e}$ pertencentes a probabilidade conjunta é uma inferência.

A inferência em uma rede bayesiana define-se, Colla (2007):

$P\left(X_{q} \mid X_{e}\right)=\frac{P\left(X_{q}, X_{e}\right)}{P\left(X_{e}\right)}=\frac{\sum_{X \backslash\left\{X_{q}, X_{e}\right\}} P\left(X_{n}\right)}{\sum_{X \backslash\left\{X_{e}\right\}} P\left(X_{n}\right)}$

Onde:

$P\left(X_{n}\right)=\prod_{i} P\left(X_{i} \mid\right.$ Pais de $X_{i}$ em $\left.G\right)$

,$X \backslash\left\{X_{q}, X_{e}\right\}$ são todas as variáveis de $X_{n}$ exceto as variáveis de $X_{e}$ e $X_{q}$.

,$X \backslash\left\{X_{e}\right\}$ são todas as variáveis de $X_{n}$ exceto as variáveis de $X_{e}$.

Esta abordagem para inferir em uma probabilidade conjunta é chamada de eliminação de variáveis por justamente eliminar as variáveis aleatórias da probabilidade conjunta que não são necessárias para a inferência. As variáveis restantes são as variáveis requisitadas $X_{r}$ para inferir em uma probabilidade conjunta $P\left(X_{n}\right)$, onde se questionam as variáveis $X_{q}$ que possuem evidências nas variáveis $X_{e}$, Colla (2007). 
Inferência em uma Rede Bayesiana: Método de Eliminação de Variáveis - Um exemplo

Como representação computacional de uma rede bayesiana utilizaremos um padrão chamado XMLBIF,Cozman (2016), que significa Formato Portátil de Redes Bayesianas baseado em XML.

Considere a Rede Bayesiana representada pela figura abaixo conforme exemplo criado em Colla (2007):

Tabela 2.7: Eliminação de variáveis:

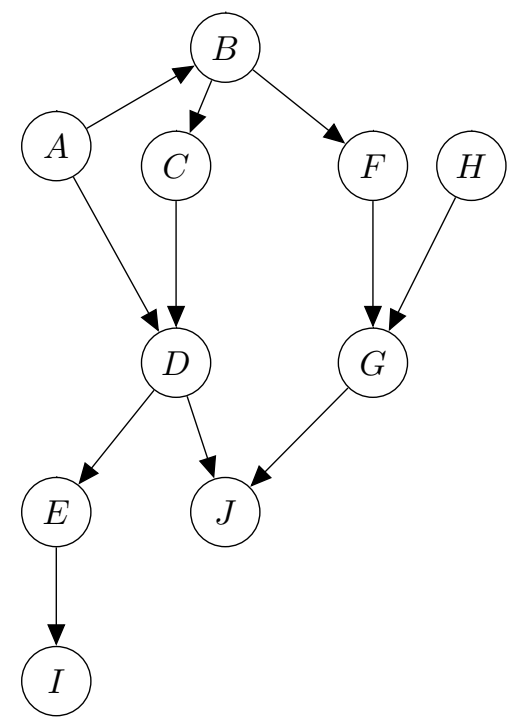


Vamos calcular a distribuição marginal a posteriori da variável $I$ considerando que foram observados os estados das variáveis $A=A 1, H=H 2$ e $J=J 3$. Ou seja, iremos obter $P(I \mid A=$ $A 1, H=H 2, J=J 3)$. As variáveis observadas e a consulta da variável em questão também serão representadas por XMLBIF.

A Rede Bayesiana é a representação da distribuição conjunta das variáveis aleatórias $X=$ $\{A, B, C, D, E, F, G, H, I, J\}$. A topologia do grafo direcionado demonstra a relação de dependência das variáveis fazendo com que as distribuições condicionais fiquem claras, assim como os seus potenciais:

$P(A)=\phi_{A}(A), P(B \mid A)=\phi_{B}(B, A), P(C \mid B)=\phi_{C}(C, B), P(D \mid A, C)=\phi_{D}(D, A, C), P(E \mid D)=$ $\phi_{E}(E, D), P(F \mid B)=\phi_{F}(F, B), P(G \mid F, H)=\phi_{G}(G, F, H), P(H)=\phi_{H}(H), P(I \mid E)=\phi_{I}(I, E), P(J \mid D, G)=$ $\phi_{J}(J, D, G)$.

Com base na Condição de Markov que demonstra uma distribuição conjunta de uma dada Rede Bayesiana, este exemplo possui a seguinte distribuição conjunta:

$P(X)=P(A) \cdot P(B \mid A) \cdot P(C \mid B) \cdot P(D \mid A, C) \cdot P(E \mid D)$

$. P(F \mid B) . P(G \mid F, H) \cdot P(H) . P(I \mid E) . P(J \mid D, G)$

Utilizando a notação de potencial para a mesma distribuição:

$P(X)=\phi_{A} \cdot \phi_{B} \cdot \phi_{C} \cdot \phi_{D} \cdot \phi_{E} \cdot \phi_{F} \cdot \phi_{G} \cdot \phi_{H} \cdot \phi_{I} \cdot \phi_{J}$

Define-se o conjunto de variáveis questionadas como $X_{q}=\{I\}$ e o conjunto das variáveis observadas como $X_{E}=\{A, H, J\}$, que são a Evidência. Dada a observação das variáveis temos a Evidência $e$ que é representada pelos findings: $e_{A}=\{1,0,0\}, e_{H}=\{0,1,0\}, e_{J}=\{0,0,1\}$.

$A=A_{1}, H=H_{2}, J=J_{3}$

Considerando a Evidência $e$, temos a distribuição conjunta de probabilidade:

$P(X, e)=\phi_{A} \cdot e_{A} \cdot \phi_{B} \cdot \phi_{C} \cdot \phi_{D} \cdot \phi_{E} \cdot \phi_{F} \cdot \phi_{G} \cdot \phi_{H} \cdot e_{H} \cdot \phi_{I} \cdot \phi_{J} \cdot e_{J}$

Para termos a distribuição marginal a posteriori da variável $I$ deve-se marginalizar todas as variáveis exceto $I$. Distribuição posteriori significa que é uma distribuição após a observação de eventos relevantes para a distribuição conjunta, como a Evidência $e$. E distribuição marginal de $R$ é a distribuição de uma única variável $R$ obtida através da distribuição conjunta na qual esta variável $R$ faz parte.

$P(I, e)=\sum_{(A, B, C, D, E, F, G, H, J)} \phi_{A} \cdot e_{A} \cdot \phi_{B} \cdot \phi_{C} \cdot \phi_{D} \cdot \phi_{E} \cdot \phi_{F} \cdot \phi_{G} \cdot \phi_{H} \cdot e_{H} \cdot \phi_{I} \cdot \phi_{J} \cdot e_{J}$

A distribuição da variável consultada $I$ poderá ser obtida normalizando a distribuição marginalizada anteriormente:

$P(I \mid e)=\frac{P(I, e)}{\left(\sum_{I} P(I, e)\right)}$

Desta maneira pode-se inferir sobre a variável aleatória $I$.

\subsection{Variáveis Requisitadas}

Em uma Rede Bayesiana para se inferir sobre uma variável $X_{q}$ não necessariamente precisa-se consultar todas as variáveis da rede. Para selecionar somente as variáveis requisitadas $X_{R}$ para a inferência, existe o algoritmo Bayes-Ball, Colla (2007).

Faz-se analogia de maneira que cada vértice possui uma parte inferior e superior e de que bolas irão percorrer o grafo. A marcação dos nós segue a seguinte lógica:

- A parte superior do nó deve ser marcada quando o mesmo enviar a bola para seus pais;

- A parte inferior do nó deve ser marcada quando o mesmo enviar a bola para seus filhos;

- Um nó observado deve ser assinalado (checked) quando for visitado.

Resumindo e consolidando as regras de movimentações e marcação dos nós conforme figura a seguir:

1. Um nó probabilístico não observado:

(a) Visitado a partir de um de seus pais: enviar a bola para todos seus filhos e marcar a parte inferior. 
(b) Visitado a partir de um de seus filhos: enviar a bola para todos seus pais e marcar a parte superior, enviar a bola para todos seus filhos e marcar a parte inferior.

2. Um nó probabilístico observado:

(a) Visitado a partir de um de seus pais: assinalar o nó, enviar a bola para todos seus pais e marcar a parte superior.

(b) Visitado a partir de um de seus filhos: assinalar o nó e interromper a movimentação da bola.

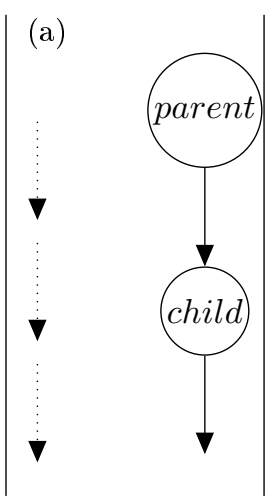

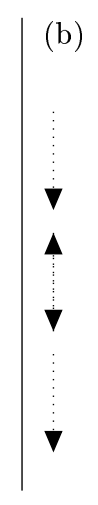

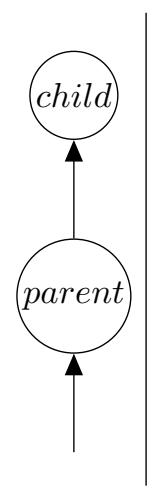

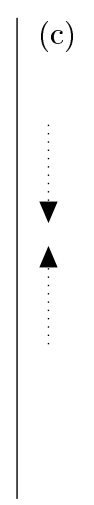
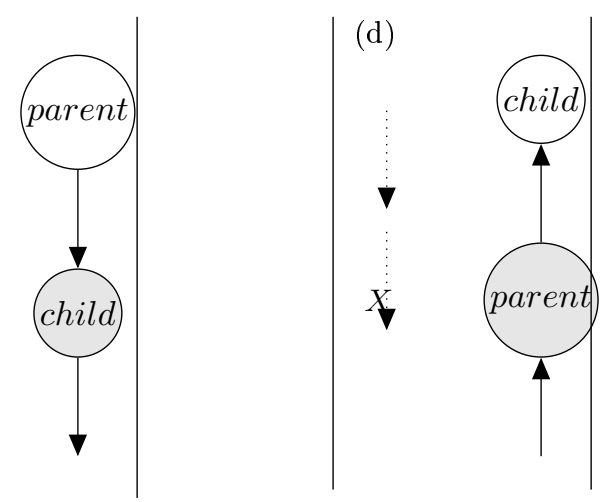

Tabela 2.8: Variáveis Requisitadas.

Considere uma Rede Bayesiana composta por um conjunto de variáveis $X=\left\{X_{1}, \ldots, X_{n}\right\}$, da qual se deseja calcular $P\left(X_{q} \mid E\right)$. O algoritmo descrito explora o grafo da Rede Bayesiana e constrói $X_{R}$ :

1. Inicialize todos os nós do grafo como não visitados e não marcados na parte superior e inferior.

2. Crie um programa de nós a serem visitados, inicializando com cada nó de $X_{q}$ como se o mesmo fosse visitado a partir de um nó filho virtual.

3. Enquanto tiver nós para serem visitados:

(a) Pegue um nó i programado para ser visitado e retire-o do programa. O nó i pode estar programado para ser visitado por um pai, ou para ser visitado por um filho ou por ambos.

(b) Marcar o nó i como visitado.

(c) Se o nó $i \notin E$ e a visita é de um filho: Se a parte superior de i não estiver marcada, marque-a e programe cada um de seus pais para serem visitados; Se a parte inferior de i não estiver marcada, marque-a e programe cada um de seus filhos para serem visitados.

(d) Se a visita a i é de um pai: Se $i \in E$ e a parte superior de i não estiver marcada, marque-a e programe cada um de seus pais para serem visitados; Se $i \notin E$ e a parte inferior de i não estiver marcada, marque-a e programe cada um de seus filhos para serem visitados.

4. Os nós irrelevantes são os nós não marcados na parte inferior.

5. Os nós probabilísticos requisitados são os nós marcados na parte superior

6. Os nós observados requisitados são os nós marcados pertencentes a E marcados como visitados

7. O conjunto $X_{R}$ será a união dos conjuntos de nós probabilísticos e observados requisitados. 


\subsection{Inferência em Redes Credais}

E estendendo a definição da eliminação de variáveis de redes bayesianas para inferir em uma rede credal, o conjunto credal de probabilidades condicionais das variáveis questionadas dada a ocorrência das variáveis de evidência pode ser calculado conforme abaixo:

$$
\begin{aligned}
& K\left(X_{q} \mid X_{e}\right)=\frac{K\left(X_{q} \cap X_{e}\right)}{K\left(X_{e}\right)} \\
& =\frac{\sum_{X \backslash\left\{X_{q}, X_{e}\right\}}^{\otimes} K\left(X_{n}\right)}{\sum_{X \backslash\left\{X_{e}\right\}}^{\otimes} K\left(X_{n}\right)}
\end{aligned}
$$

E para que somente as variáveis de evidência sejam utilizadas, a marginalização das outras variáveis dentro da probabilidade conjunta se faz necessária através da fórmula abaixo:

$\sum_{X \backslash\left\{X_{e}\right\}}^{\otimes} K\left(X_{n}\right)$

O mesmo acontece para as variáveis questionadas:

$\sum_{X \backslash\left\{X_{q}, X_{e}\right\}}^{\otimes} K\left(X_{n}\right)$

Esta técnica de inferência chamada eliminação de variáveis é a aplicação da regra de bayes à rede credal, considerando somente as variáveis requisitadas.

E conforme a fórmula acima, o conjunto credal da probabilidade conjunta de toda a rede credal representada por $K\left(X_{n}\right)$ se faz necessário. E para chegar neste conjunto credal da probabilidade conjunta de toda a rede credal é que a técnica de eliminação de variáveis entra em ação. Conforme foi definido pela condição de markov, o conjunto credal da probabilidade conjunta é definido como sendo:

$$
K\left(X_{n}\right)=\otimes_{i} K\left(X_{i} \mid \text { Pais de } X_{i} \text { em } G\right)
$$

Onde o operador composição $\otimes$ vai adicionando variáveis ao conjunto credal da probabilidade conjunta que serão marginalizadas ou eliminadas por não fazerem parte das variáveis requisitadas Antonucci et al. (2014).

\subsection{Grafo Moral}

O Grafo Moral $M(G)$ de um grafo direcionado acíclico $G$ é um grafo não direcionado com o mesmo conjunto de nós de $G$, arcos não direcionais unindo os nós que estavam unidos em $G$ e adicionalmente contendo arcos não direcionais unindo os nós $i$ e $j$ se eles forem parentes imediatos em $G$.

O conjunto dos parentes imediatos de um nó $i$ no grafo $G$ inclui seus pais, $p a(i)$, seus filhos, $c h(i)$, e seus esposos, $s p(i)$. A este conjunto de parentes imediatos denomina-se Markov Blanket de $i, m b(i)$. Se $j$ pertence a $m b(i)$ então $j$ será um nó adjacente a $i$ no Grafo Moral. A figura abaixo apresenta o grafo direcionado (a) como exemplo, seu Grafo Moral (b) e o Markov Blanket para o nó $V_{4},(\mathrm{c})$. Os arcos não direcionados adicionais incorporados ao Grafo Moral são usualmente denominados de arcos morais (moral links) pois conectam dois nós com um ou mais filhos em comum, Colla (2007), Koller e Friedman (2009). 

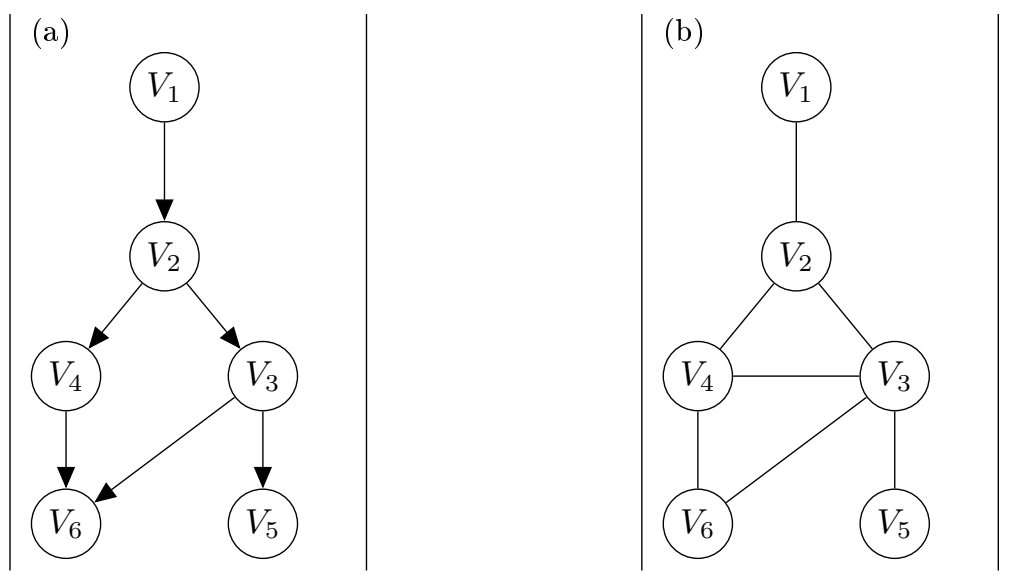

Tabela 2.9: Grafo Moral.

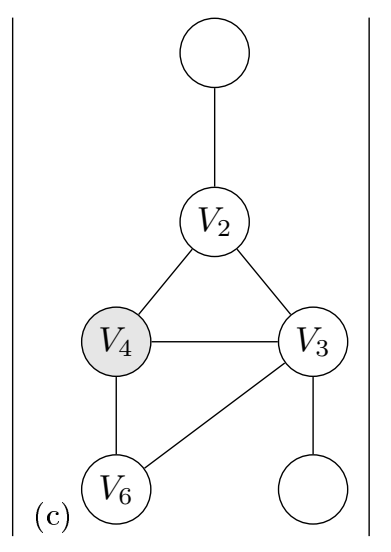

\subsection{Grafo Preenchido}

A ordenação de um conjunto de vértices $V=\left\{v_{1}, v_{2}, \ldots, v_{n}\right\}$ de um grafo pode ser descrita utilizando um vetor de índices q. $\mathrm{O}$ vetor de índices $q=[1,2, \ldots, N]$ representa a ordem natural dos nós. A partir de uma permutação $\sigma$ da ordem natural $[1,2, \ldots, N]$ pode-se obter uma nova ordenação, a qual será indicada pelo vetor $q=[\sigma(1), \sigma(2), \ldots, \sigma(N)]$ ou, quando não houver ambiguidade, simplesmente por $q=[(1),(2), \ldots,(N)]$, Colla $(2007)$.

Define-se o processo de eliminação em um grafo não direcionado $G=(V, E)$, para uma dada uma ordem, $q=[(1),(2), \ldots,(N)]$, como uma sequência de grafos de eliminação $G_{k}=\left(V_{k}, E_{k}\right)$ na qual, para $k=1,2, \ldots, n$, tem-se:

$$
\begin{aligned}
& V_{k}=V_{q(k)}, V_{q(k+1)}, \ldots, V_{q(n)} \\
& E_{1}=E
\end{aligned}
$$

para $k>1,\{i, j\} \in E_{k}$ ou $\{q(k-1), i\},\{q(k-1), j\} \in E_{(k-1)}$

A ordem $q=[(1),(2), \ldots,(N)]$ será denominada de ordem de eliminação na qual $q(k)=i$ significa que o vértice $i$ foi o k-ésimo vértice de $\mathrm{G}$ a ser eliminado.

Ao eliminar o vértice $q(k)$, os arcos não direcionados adicionados para unir seus vizinhos são denominados de arcos de preenchimento. A inclusão de todos os arcos de preenchimento unindo os vizinhos de $q(k)$ transforma-os em um clique. Uma ordem de eliminação, $q$, será perfeita se e somente se a eliminação de nenhum dos vértices na ordem $q$ acarreta a incorporação ao grafo de um arco de preenchimento.

A partir do processo de eliminação é possível construir o Grafo Preenchido $P=(V, F)$, onde $F=\cup_{(k=1)}^{n} E_{k}$. Considerando que o grafo originalmente possuía $\mathrm{E}$ arcos, os arcos de preenchimento correspondem à diferença $F-E$.

A figura abaixo apresenta um grafo original com 6 vértices (a), e uma sequência de grafos de 
eliminação (b) a (e). Em cada etapa está em destaque o nó que será eliminado e na etapa seguinte os arcos de preenchimento adicionados. A figura (f) mostra o grafo preenchido, novamente com os arcos adicionados em destaque. Neste exemplo a ordem de eliminação adotada corresponde ao vetor $q=[1,3,6,2,4,5]$.
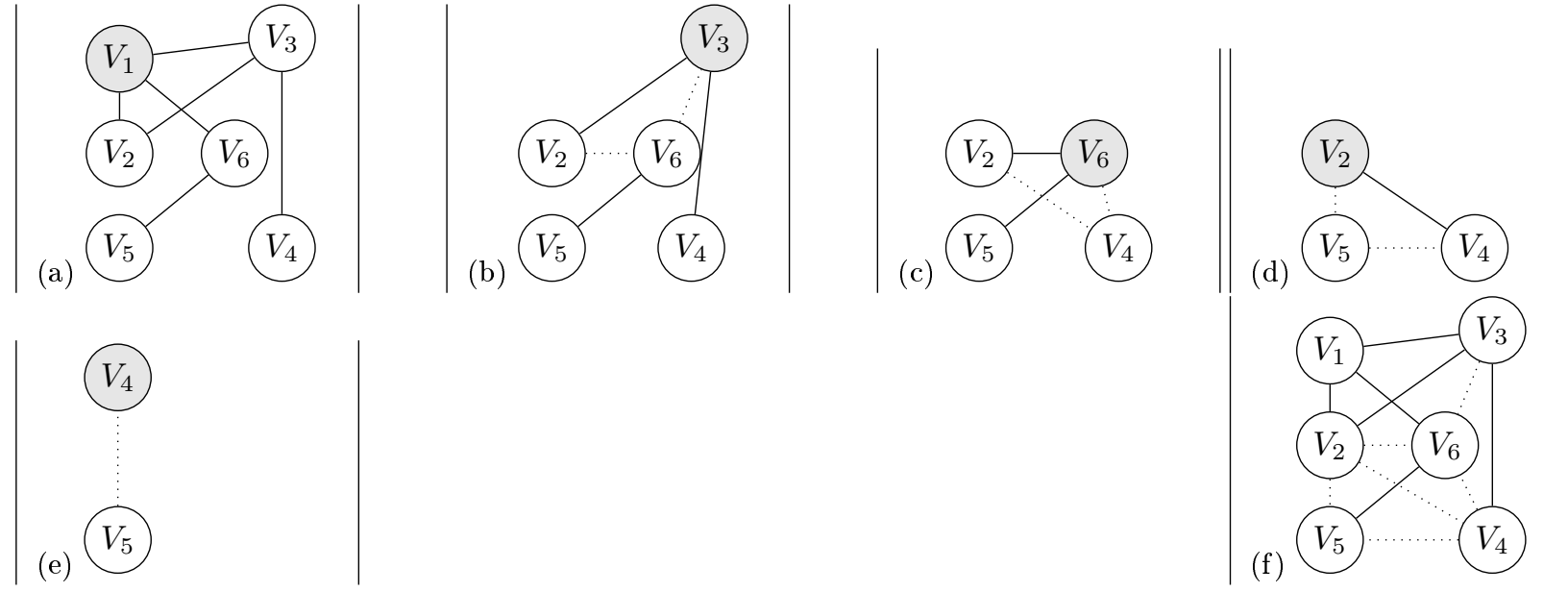

Tabela 2.10: Grafo Preenchido. 


\section{Capítulo 3}

\section{Inferência em Redes Credais usando técnicas de Âlgebra Linear (Fatoração Simbólica)}

\subsection{Fatoração de Matrizes aplicada na inferência de redes bayesia- nas}

O algoritmo paralelo de inferência em redes bayesianas pode ser dividido em duas partes conforme explica Colla (2007), o criador do algoritmo:

- Fatoração Simbólica: Nesta fase, em uma rede bayesiana são definidos os conjuntos das variáveis requisitadas, a ordem de eliminação e a Árvore de Eliminação;

- Fatoração Numérica: Nesta fase ocorrem os cálculos numéricos que incluem as operações de produto, marginalização de variáveis e normalização envolvendo os potenciais da Rede Bayesiana.

A Fatoração Simbólica constitui a contribuição mais importante do trabalho de Colla (2007) pois o mesmo resulta da aplicação para inferência em Redes Bayesianas de técnicas de Álgebra Linear Computacional para fatoração de matrizes esparsa. A Fatoração Simbólica consiste na determinação da ordem de eliminação e na construção da Árvore de Eliminação dos vértices do grafo da Rede Bayesiana. O objetivo de gerar estas duas estruturas de dados é possibilitar a paralelização e a otimização do processamento numérico necessário para inferência. Convêm ressaltar que os algoritmos utilizados na Fatoração Simbólica tanto para a determinação da ordem de eliminação como para a construção da Árvore de Eliminação utilizam basicamente o Grafo Moral gerado a partir da Rede Bayesiana.

A denominação Fatoração Simbólica foi adotada em analogia ao processo de Fatoração de Cholesky de matrizes esparsas. No caso da Fatoração de Cholesky, este algoritmo é o mesmo procedimento para a determinação da Árvore de Eliminação, uma vez definido o grafo não direcional que representa a matriz, ocorre sem a necessidade de utilizar os valores numéricos da matriz.

\subsection{Fatoração de Matrizes aplicada na inferência de redes credais}

O algoritmo paralelo utilizado nesta tese para inferir em uma rede credal possui também duas fases assim como o algoritmo de inferência da rede bayesiana Colla (2007): a fase simbólica e a fase numérica.

- Fatoração Simbólica: Esta fase faz exatamente como a fatoração simbólica da rede bayesiana, onde as variáveis requisitadas, a ordem de eliminação e a Árvore de Eliminação são definidas. 
Pois a única informação necessária para esta fase será o grafo acíclico da rede credal, que é exatamente a mesma informação que uma rede bayesiana forneceria;

- Fatoração Numérica: Nesta fase ocorrem os cálculos numéricos que diferem da rede bayesiana e são específicos de conjuntos credais como as operações de eliminações das variáveis. E as operações definidas no capítulo anterior que são necessárias para executar a regra de bayes em conjuntos credais após as eliminações das variáveis.

\subsection{Fatoração Simbólica}

\subsubsection{Fatoração de Cholesky}

A Fatoração Simbólica de uma Rede Bayesiana, que utiliza para construção da Árvore de Eliminação é exatamente o mesmo algoritmo que a Fatoração de Cholesky em questão, e tem como base apenas a topologia do Grafo Moral associado a mesma.

Conforme figura abaixo, um grafo não direcionado $G=(V, E)$ com $n$ vértices está associada uma matriz de adjacência ${ }_{0} A n \times n$ e, portanto, cada vértice $i$ do grafo está associado a uma coluna em ${ }_{0} A$.

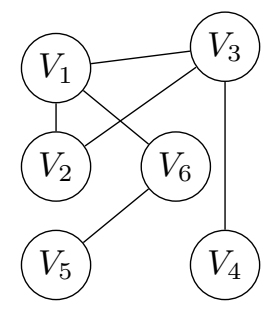

Tabela 3.1: Grafo direcionado - Fatoração Cholesky

$$
\left(\begin{array}{llllll}
1 & x & x & & & x \\
x & 2 & x & & & \\
x & x & 3 & x & & \\
& & x & 4 & & \\
& & & & 5 & x \\
x & & & & x & 6
\end{array}\right)
$$

Tabela 3.2: Matriz adjacência - Fatoração Cholesky

Para uma determinada ordem de eliminação $q=[(1),(2), \ldots,(N)]$, obtém-se uma sequência de grafos de eliminação $G_{k}=\left(V_{k}, E_{k}\right)$ para $k=1,2, \ldots, n$, na qual $q(k)=i$ significa que o vértice i foi o k-ésimo vértice de $G$ a ser eliminado. A cada grafo $G_{k}$ está associada uma matriz de adjacência transformada ${ }_{k} A$ resultado da anulação na matriz ${ }_{k-1} A$ dos elementos abaixo da diagonal principal da coluna associada ao vértice $i$.

Os grafos de eliminação $G_{k}$ são grafos cujas matrizes de adjacência são as matrizes transformadas ${ }_{k} A$ do processo de fatoração. Esta associação implica que ao eliminar a j-ésima coluna da matriz na Fatoração de Cholesky serão preenchidas na matriz (com zeros) exatamente as posições correspondentes aos arcos de preenchimento adicionados ao grafo preenchido quando da eliminação do vértice $q(j)$. 
Analogamente à eliminação de vértices em um grafo, dir-se-á que a ordem de eliminação, q, é perfeita quando não ocorrer o preenchimento de nenhuma posição ao longo do processo de eliminação das colunas na Fatoração de Cholesky.

A figura abaixo ilustra a sequência de grafos de eliminação para a permutação representada pela matriz da figura a seguir.
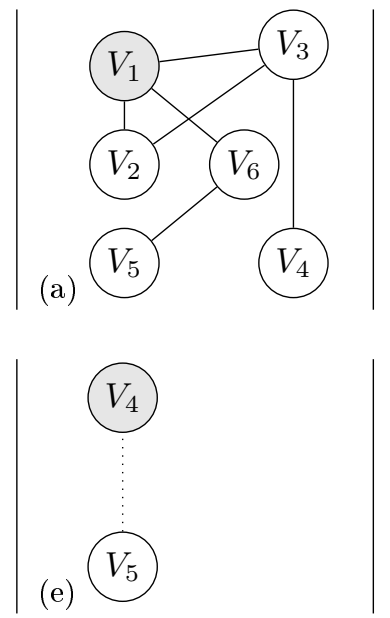

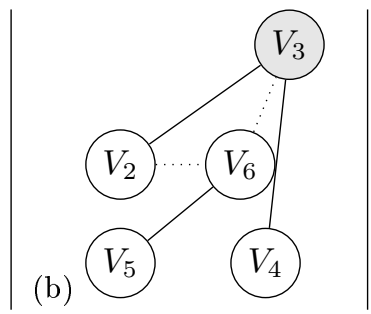

(c)
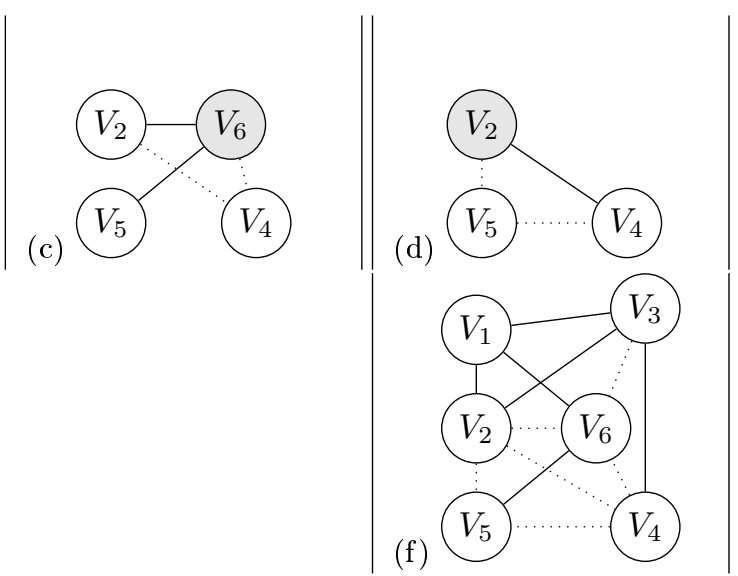

Tabela 3.3: Grafo Preenchido.

$$
\left(\begin{array}{llllll}
1 & x & x & x & & \\
x & 3 & & x & x & \\
x & 0 & 6 & 0 & 0 & x \\
x & x & 0 & 2 & 0 & 0 \\
& x & 0 & 0 & 4 & 0 \\
& & x & 0 & 0 & 5
\end{array}\right)
$$

Tabela 3.4: Fatoração de Cholesky - Matriz do Grafo Preenchido.

\subsubsection{Ordem de Eliminação}

Esta seção tem como objetivo descrever a aplicação das Heurísticas de Busca em Largura e da Heurística de Gibbs para se obter uma boa ordenação para eliminação dos vértices. Conforme procurou-se deixar claro nas seções anteriores, a ordem de eliminação das variáveis é fundamental para garantir que o processo seja computacionalmente eficiente. No entanto, até o presente momento adotou-se a ordem de eliminação dos vértices de um grafo ou, o seu análogo para matrizes, a ordem de eliminação das colunas como conhecida. Preencher esta lacuna é o objetivo desta próxima seção.

\subsection{3 Ârvore de Dissecção}

Considere um grafo $G=(V, E)$ e um separador $S$ em $G$ que divide $V-S$ em componentes conexas $C_{1}, C_{2}, C_{3}, \ldots, C_{k}$. Recursivamente para cada uma das componentes podemos considerar um separador que a divida em novas componentes conexas. Este processo pode ser representado pela 
árvore de dissecção, $D$, na qual o separador $S$ é a raiz, uma componente separada por $S$, ou um separador dentro da mesma, é filha de $S$ e as componentes não divididas são as folhas da árvore.

Considere o grafo da figura abaixo. Utilizando inicialmente o separador $\{4,5\}$ divide-se o grafo $G$ nas componentes conexas $C 1=\{1,3,8,9,10\}, C 2=\{11\}$ e $C 3=\{2,6,7,12,13\}$, conforme indicado na figura a seguir.

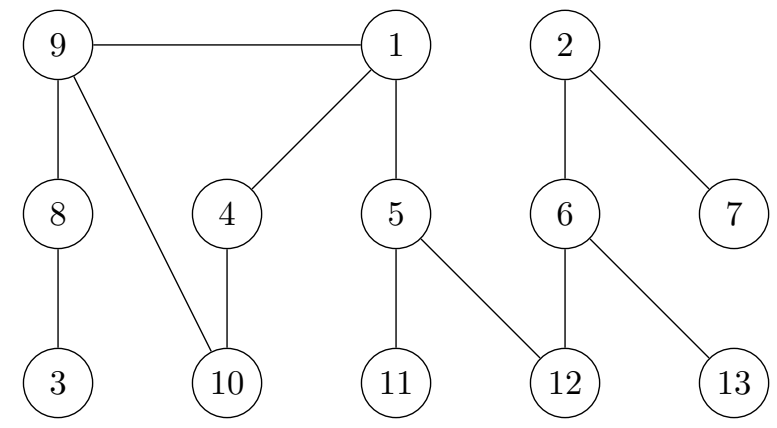

Tabela 3.5: Confecção da Árvore de dissecção - Passo 1

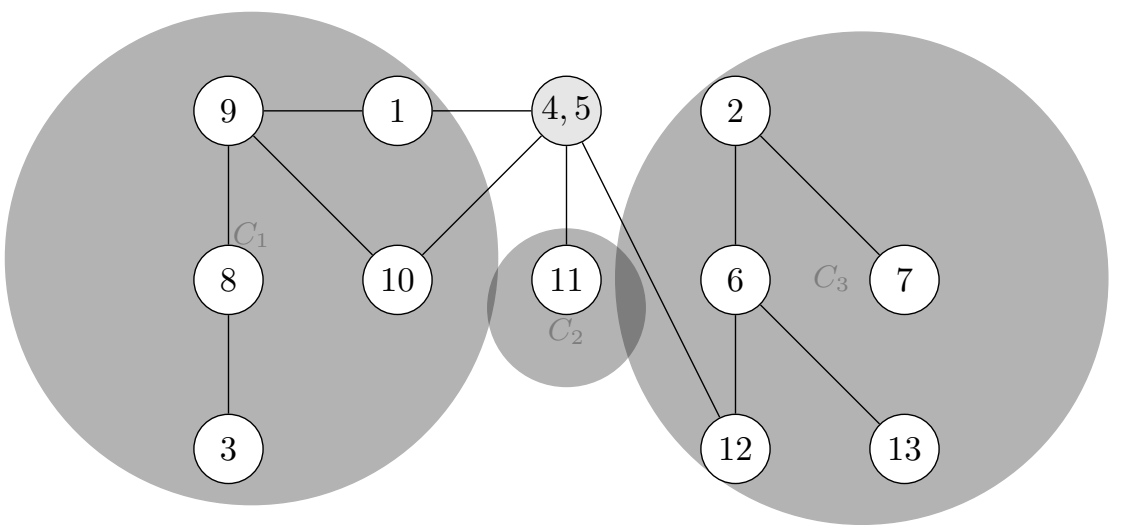

Tabela 3.6: Confecção da Árvore de dissecção - Passo 2

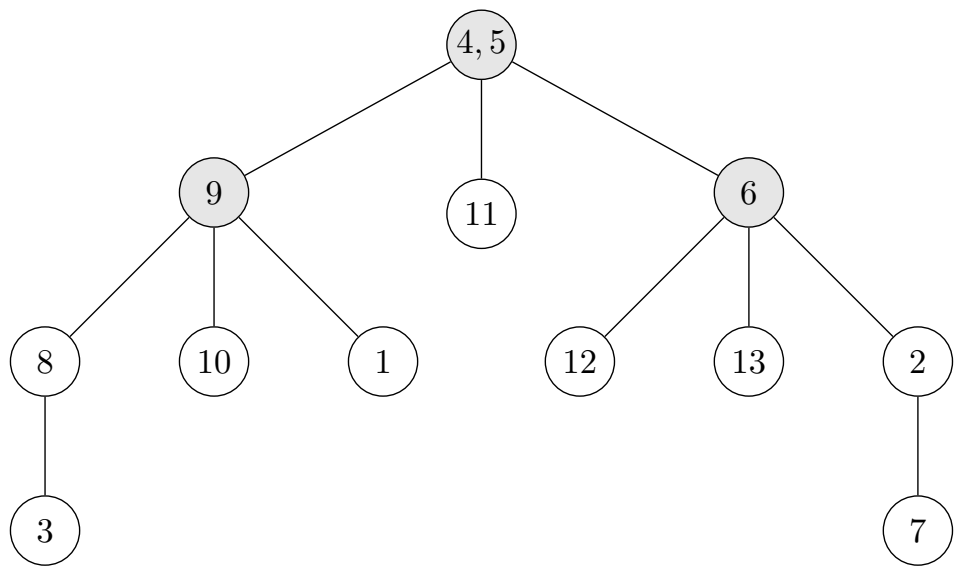

Tabela 3.7: Confecção da Árvore de dissecção - Passo 3 
Dada uma árvore $H$ de raiz $r$, uma pós-ordem, $\sim q$, dos vértices de $H$ é uma ordem que enumera os vértices de cada uma as árvores $H-r$, recursivamente em pós-ordem e ao final a raíz $r$. Sendo $q$ uma pós-ordem da árvore de dissecção $D$ gerada a partir de um grafo $G$, a ordem de dissecção, $q$, é obtida substituindo em $q$ cada nó, $d$, da árvore de disseção pelos vértices correspondentes do grafo original $G$ que compõe d. Uma possível ordem de dissecção para a árvore de dissecção, $D$, representada pela figura acima seria $q=[3,8,1,10,9,12,13,2,7,6,11,4,5]$.

Considere uma árvore de dissecção, $D$, de nós $d$, gerada a partir de um grafo $G=(V, E)$ com $v \in V$ e uma na ordem de dissecção $q$. Pode-se demonstrar Stern (1994) que a eliminação de um dado vértice $v \in d$, pode gerar apenas o inclusão de arcos de preenchimento entre os vértices de $G$ pertencentes ao nó $\mathrm{d}$, ou entre vértices de d e vértices em nós ancestrais de $d$ em $D$ ou ainda entre vértices em ascendentes de $d$.

Para se obter, para um grafo qualquer, bons separadores que posteriormente determinem uma ordem de eliminação que minimize o surgimento de arcos de preenchimento, ou o preenchimento de posições na matriz, bem como gerem Árvores de Eliminação que possibilitem a paralelização das operações, podem ser utilizadas a Heurística de Busca em Largura (BEL) e a Heurística de Gibbs Pissanetzky (2005) e Stern (2006).

\subsubsection{Heurística de Busca em Largura e a Heurística de Gibbs}

Para a determinação da ordem de eliminação adotou-se a Heurística de Busca em Largura (BEL) e a Heurística de Gibbs. Cabe mencionar que existem formas alternativas para se determinar a ordem de eliminação, no entanto, as heurísticas adotadas são particularmente interessantes pois estão estreitamente relacionadas com a etapa seguinte da Fatoração Simbólica na qual será gerada a Árvore de Eliminação. O que torna as heurísticas adotadas interessantes para o algoritmo que será proposto é o fato das mesmas buscarem simultaneamente dois objetivos principais:

- Gerar uma ordem eficiente de eliminação, o que basicamente significa minimizar a inserção de arcos de preenchimento à medida que os vértices do grafo são eliminados;

- Gerar uma ordem que maximize as possibilidades de paralelização do processamento numérico.

O algoritmo descrito abaixo é apenas uma sistematização teórica das heurísticas aplicada ao Grafo Moral de uma Rede Bayesiana.

\section{Heurística da Busca em Largura}

Dado um grafo $G=(V, E)$, adotando um vértice $v \in V$ como raiz particione os vértices de $G$ em níveis $L_{0}, L_{1}, \ldots . L_{k}$, definidos por:

- $L_{0}=\{v\}, L_{(i+1)}=\operatorname{adj}\left(L_{i}\right)-L_{(i-1)}$

- A profundidade do nível $L_{i}$ é i, e a largura do nível $L_{i}$, definida como o número de elementos do nível $L_{i}$, é \# $L_{i}$. A máxima profundidade e a máxima largura da BEL são respectivamente a sua profundidade e a sua largura.

- O nível $L_{i}$ separa em $G$ os vértices em níveis mais profundos dos vértices em níveis menos profundos que $i$ Colla (2007). A heurística de BEL procura um separador balanceado $S \subseteq L_{i}$ tomando $i \approx k / 2$, ou então tomando:

- $i \mid \sum_{j=0}^{i-1} \# L_{j} \leq \frac{n}{2} \wedge \sum_{(j=i+1)}^{k} \# L_{j} \leq \frac{n}{2}$

Para reduzir a largura da BEL, com o objetivo de se obter separadores pequenos, a heurística procura para raiz um vértice $v \in V$ que gere uma BEL de máxima profundidade.

No grafo $\mathrm{G}$ a distância de um vértice v a um vértice $w$, representa-se por $\operatorname{dist}(v, w)$, é número de arcos do caminho mais curto entre ambos os vértices. A excentricidade de um vértice vé exc $(v)=$ $\max _{(w \in v)} \operatorname{dist}(v, w)$. Um vértice de máxima excentricidade se diz periférico, e sua excentricidade é o diâmetro de $G$. 
Uma BEL cuja raiz seja v terá a profundidade igual a excentricidade de v. Desta forma, com o intuito de gerar a BEL de máxima profundidade é desejável que a raiz da BEL seja um vértice periférico. No entanto, encontrar um vértice periférico é um problema computacionalmente difícil.

Para contornar o problema de selecionar um vértice periférico adotou-se a Heurística de Gibbs para encontrar um vértice quase-periférico, conforme pode ser visto em Colla (2007).

\section{Heurística de Gibbs}

Dado um grafo $G=(V, E)$ :

1. Escolher como raiz um vértice de grau mínimo 1

2. Forme os níveis da BEL com raiz $v, L_{0}, L_{1}, \ldots, L_{k}$, particione o nível mais profundo em suas 1 componentes conexas, $l_{k}=\cup_{(j=1)}^{l}$, e tome um vértice de grau mínimo, $v_{j}$, em cada componente.

3. Para $\mathrm{j}=1: 1$ Tome $v_{j}$ como nova raiz e encontre os níveis de BEL $L_{0}, L_{1}, \ldots . L_{k}^{\prime}$ Até que $k^{\prime}>k$ ou $j=l$

4. Se o passo 3 terminou com $k^{\prime}>k$, volte ao passo 2. Caso contrário a atual raiz é um vértice quase-periférico.

Retomando o grafo e a árvore de dissecção estudados:

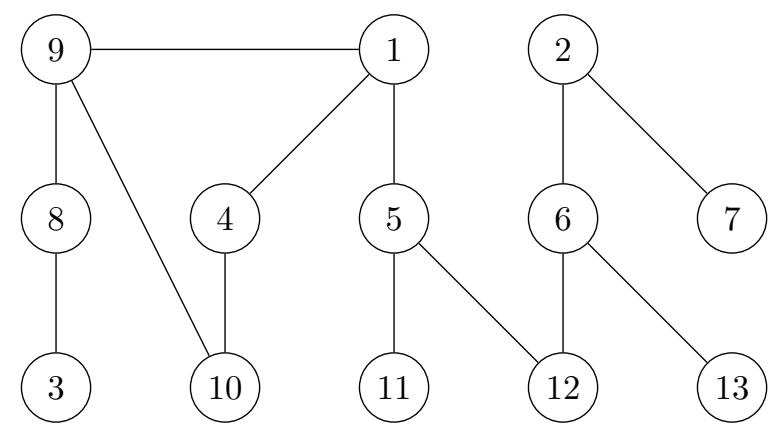

Tabela 3.8: Grafo e Árvore de dissecção 
A heurística de Gibbs encontra 3 como vértice quase-periférico. Tomando 3 como raiz gera-se a árvore $H$ por BEL:

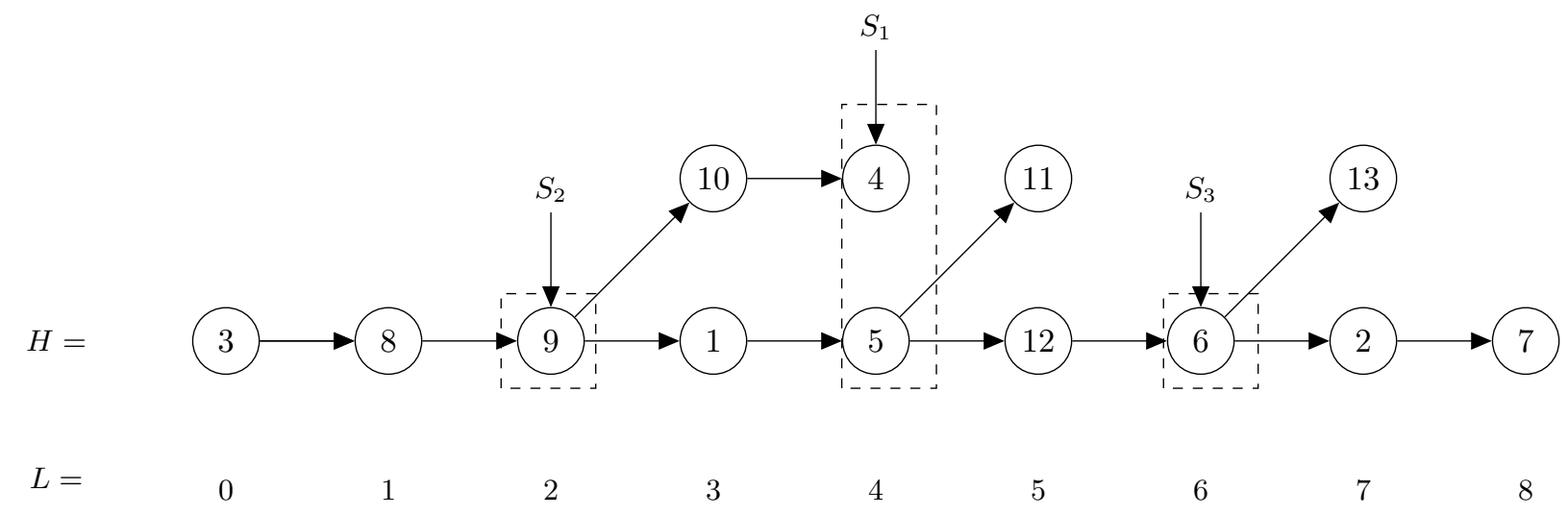

Tabela 3.9: Árvore BEL-Gibbs

Utilizando inicialmente o separador $S 1=\{4,5\}$ obtêm-se as componentes conexas $C 1=$ $\{1,3,8,9,10\}, C 2=\{11\}$ e $C 3=\{2,6,7,12,13\}$. Em seguida, para a componente $C 1$ escolhese o separador $S 2=\{9\}$ e para a componente $C 3$ o separador $S 3=\{6\}$. Obtêm-se portanto a árvore de dissecção da figura abaixo.

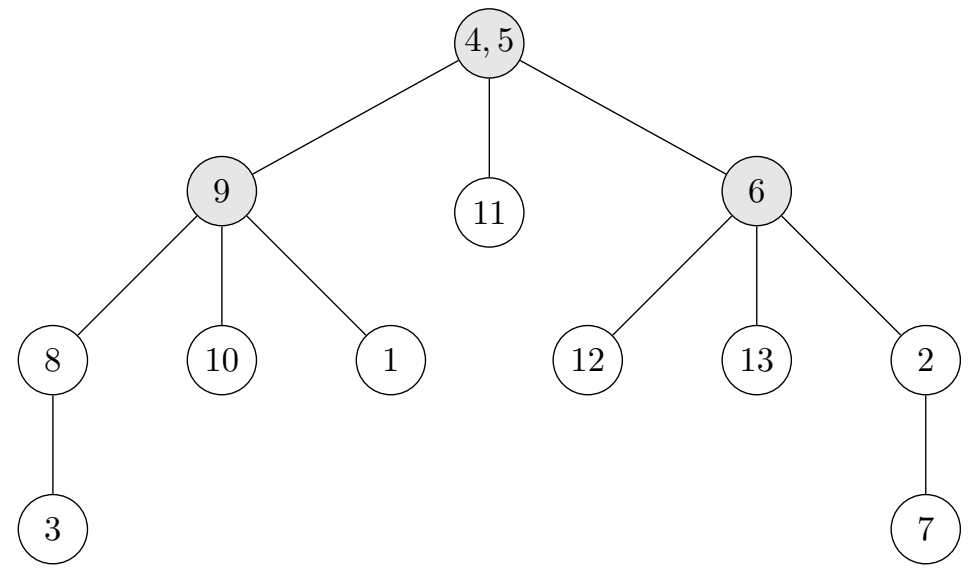

Tabela 3.10: Árvore de dissecção 
Listando-se os nós da árvore de dissecção assim obtida em pós-ordem obtêm-se a ordem de dissecção $q=[3,8,1,10,9,12,13,2,7,6,11,4,5]$.

\section{Algoritmo para Determinação da Ordem de Eliminação}

Dado um Grafo Moral $G=(V, E)$ associado a uma Rede Bayesiana:

1. Inicialize todos os vértices do grafo como não testados.

2. Escolha um vértice de grau mínimo v do grafo e marque-o como testado.

3. Construa a BEL adotando como raiz o vértice v e a defina como a BELcorrente.

4. Construa uma lista de nós a serem testados com os nós do nível mais profundo da BELcorrente.

5. Enquanto a lista de nós a serem testados não for vazia:

(a) Para cada nó v da lista de nós a serem testados faça: Marque o nó v como testado. Construa a BEL adotando como raiz o vértice v e a defina como BELnova. Se, segundo os critérios de comparação, a BELnova for superior a BELcorrente então adote a BELnova como a nova BELcorrente.

(b) Se houve a substituição da BELcorrente construa uma nova lista de nós a serem testados com os nós não marcados como testados do nível mais profundo da BELcorrente.

6. Para a BELcorrente construa a Árvore de Dissecção.

7. A partir da raiz percorra a Árvore de Dissecção recursivamente montando a ordenação do vértices em pós-ordem.

8. Monte a ordem de eliminação percorrendo a lista dos vértices da Árvore de Dissecção listados em pós-ordem substituindo cada vértice da Árvore de Dissecção pelos vértices correspondentes do grafo original.

\subsection{5 Árvore de Eliminação}

Definida a ordem de eliminação, a etapa seguinte da Fatoração Simbólica da qual resultam a Árvore de Eliminação e o Grafo Preenchido foi implementada utilizando o algoritmo de eliminação simplificada Colla (2007). O algoritmo de eliminação simplificada é computacionalmente mais eficiente pois ao longo do processo de eliminação dos vértices adiciona-se ao grafo apenas os arcos de preenchimento que incidem sobre o vértice vizinho mais próximo de ser eliminado. Os arcos existentes unindo os vértices do grafo inicial, bem como os arcos de preenchimento inseridos ao longo da sequência de grafos de eliminação associada ao processo de eliminação dos vértices, expressam a relação de dependências entre as operações de marginalização de variáveis na Rede Bayesiana. Da mesma forma que na Fatoração de Cholesky os arcos mencionados expressam a relação de dependência na eliminação das colunas da matriz. A identificação destas relações de dependência é a base para a construção da Árvore de Eliminação e para a paralelização do processamento numérico. A partir das relações identificadas é possível estabelecer quais operações são independentes entre si e portanto poderão ser executadas paralelamente.

\section{Algoritmo de Fatoração Simbólica para Construção da Árvore de Eliminação}

Dado um Grafo Moral $G=(V, E)$ associado a uma Rede Bayesiana e uma ordem de eliminação $q$ :

1. Inicializar com os nós de $G$ a estrutura para armazenar a Árvore de Eliminação $H$, mas sem estabelecer ainda os arcos de $H$. 
2. Inicializar com os nós e os arcos de $G$ a estrutura para armazenar o Grafo Preenchido $F$, ligando em $F$ apenas os nós que estejam ligados em $G$.

3. $j=0$;

4. Enquanto $j<$ (Número de Vértices de $G-1$ ) faça

(a) Definir $v_{j}$ como o $j$-ésimo nó de $q$;

(b) $i=j+1$;

(c) Enquanto $i<$ (Número de Vértices $G$ ) faça

i. Definir $v_{i}$ como o $i$-ésimo nó de $q$;

ii. Se o existe em $F$ o arco $v_{j}, v_{i}$ então Acrescentar em $H$ um arco direcional $\left\{v_{i}, v_{j}\right\}$; Definir $v_{i}$ como o vizinho de $v_{j}$ mais próximo de ser eliminado; Interromper o loop e as iterações de $i$;

iii. Incrementar $i=i+1$;

5. $k=i+1$;

6. Enquanto $k<($ Número de Vértices $G$ ) faça

(a) Definir $v_{k}$ como o $k$-ésimo nó de $q$;

(b) Se existir em $F$ um arco $\left\{v_{j}, v_{k}\right\}$ e não existir em $F$ o arco $\left\{v_{i}, v_{k}\right\}$ então Acrescentar em $F$ o arco $\left\{v_{i}, v_{k}\right\}$.

(c) Incrementar $k=k+1$;

7. Incrementar $j=j+1$;

Descrito o processo de Fatoração Simbólica observe que realmente não foi utilizado em nenhum momento nenhuma informação numérica relativa às distribuições de probabilidade ou estados das variáveis da Rede Bayesiana. Isto significa que, mantidos constantes os elementos dos conjuntos como $X_{q}, X_{E}$ e a estrutura da rede, é possível fazer inúmeros exercício de inferência alterando os estados de $X_{E}$ ou mesmo as distribuições de probabilidades das variáveis sem que para isto seja necessário refazer a Fatoração Simbólica, ou seja, não é necessário refazer o processo para obter a ordem de eliminação e a Árvore de Eliminação.

\subsubsection{Inferências em Rede Credal - Exemplo}

Com a árvore de eliminação definida, foi definido quais variáveis podem ser eliminadas em paralelo e quais as dependências entre as variáveis faz com que seja necessário esperar. Para exemplificar a inferência em uma rede credal, vai ser utilizada um exemplo de rede credal definida no apêndice A. Onde esta rede credal representa o conjunto credal de probabilidades conjuntas $K(S, D, C, B)$ das variáveis aleatórias $S, D, C$ e $B$. Abaixo segue o grafo acíclico que faz parte desta rede credal: 
Tabela 3.11: Exemplo de Rede Credal do apêndice A

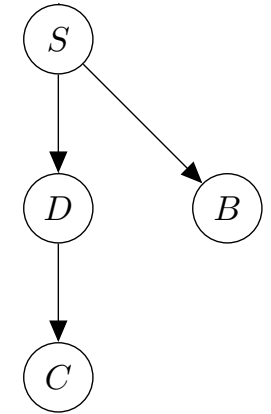

Dado o grafo acíclico apresentado pela rede credal e a condição de Markov, pode-se representar o conjunto credal de probabilidades conjuntas através dos conjuntos credais das probabilidades condicionais definidas nas redes:

$K(S, D, C, B)=K(C \mid D) \otimes K(D \mid S) \otimes K(B \mid S) \otimes K(S)$

Com esta representação os intervalos probabilísticos podem ser definidos de maneira mais simplificada:

- $K(C \mid D)$

- $\left\{x_{1}=0.15<P\left(C_{1} \mid D_{1}\right)<x_{2}=0.4\right\}$

$-\left\{x_{3}=0.05<P\left(C_{1} \mid D_{2}\right)<x_{4}=0.1\right\}$

- $\left\{x_{5}=0.5<P\left(C_{2} \mid D_{1}\right)<x_{6}=0.8\right\}$

- $\left\{x_{7}=0.0<P\left(C_{2} \mid D_{2}\right)<x_{8}=0.0\right\}$

- $K(D \mid S)$

- $\left\{x_{9}=0.2<P\left(D_{1} \mid S_{1}\right)<x_{10}=0.3\right\}$

- $\left\{x_{11}=0.3<P\left(D_{1} \mid S_{2}\right)<x_{12}=0.55\right\}$

- $\left\{x_{13}=0.15<P\left(D_{2} \mid S_{1}\right)<x_{14}=0.5\right\}$

- $\left\{x_{15}=0.0<P\left(D_{2} \mid S_{2}\right)<x_{16}=0.0\right\}$

- $K(B \mid S)$

- $\left\{x_{17}=0.2<P\left(B_{1} \mid S_{1}\right)<x_{18}=0.3\right\}$

- $\left\{x_{19}=0.3<P\left(B_{1} \mid S_{2}\right)<x_{20}=0.55\right\}$

$-\left\{x_{21}=0.15<P\left(B_{2} \mid S_{1}\right)<x_{22}=0.5\right\}$

- $\left\{x_{23}=0.0<P\left(B_{2} \mid S_{2}\right)<x_{24}=0.0\right\}$

- $K(S)$

$-\left\{x_{25}=0.25<P\left(S_{1}\right)<x_{26}=0.5\right\}$

$-\left\{x_{27}=0.5<P\left(S_{2}\right)<x_{28}=0.75\right\}$

Neste exemplo, as constantes que representam os limites dos conjuntos credais são na quantidade de vinte e oito ao se utilizar as probabilidades condicionais para representar a probabilidade conjunta. Enquanto que se fosse necessário especificar todos os limites do conjunto credal da probabilidade conjunta são necessários trinta e dois limites para representar os conjuntos credais. Essa diferença economiza quatro limites em um total de trinta e dois especificados. Isso quer dizer que para representar o conjunto credal da probabilidade conjunta para estas quatro variáveis aleatórias 
economizou-se a especificação de doze por cento dos limites dos conjuntos com a especificção pela rede credal. Esta economia de parâmetros é permitida pela condição de Markov representada pelo grafo acíclico. E além da economia de parâmetros, as probabilidades condicionais faz com que as especificações dos parâmetros sejam feitas a um número mais restrito de variáveis. Por esta restrição na quantidade de variáveis é que o processo de descoberta dos parâmetros torna-se mais natural Koller e Friedman (2009). Segue abaixo a representação do conjunto credal da probabilidade conjunta com todos os seus limites:

- $K(S, D, C, B)$

- $\left\{x_{1}<P\left(S_{1}, D_{1}, C_{1}, B_{1}\right)<x_{2}\right\}$

- $\left\{x_{3}<P\left(S_{1}, D_{1}, C_{1}, B_{2}\right)<x_{4}\right\}$

- $\left\{x_{5}<P\left(S_{1}, D_{1}, C_{2}, B_{1}\right)<x_{6}\right\}$

- $\left\{x_{7}<P\left(S_{1}, D_{1}, C_{2}, B_{2}\right)<x_{8}\right\}$

- $\left\{x_{9}<P\left(S_{1}, D_{2}, C_{1}, B_{1}\right)<x_{10}\right\}$

- $\left\{x_{11}<P\left(S_{1}, D_{2}, C_{1}, B_{2}\right)<x_{12}\right\}$

- $\left\{x_{13}<P\left(S_{1}, D_{2}, C_{2}, B_{1}\right)<x_{14}\right\}$

- $\left\{x_{15}<P\left(S_{1}, D_{2}, C_{2}, B_{2}\right)<x_{16}\right\}$

- $\left\{x_{17}<P\left(S_{2}, D_{1}, C_{1}, B_{1}\right)<x_{18}\right\}$

- $\left\{x_{19}<P\left(S_{2}, D_{1}, C_{1}, B_{2}\right)<x_{20}\right\}$

- $\left\{x_{21}<P\left(S_{2}, D_{1}, C_{2}, B_{1}\right)<x_{22}\right\}$

- $\left\{x_{23}<P\left(S_{2}, D_{1}, C_{2}, B_{2}\right)<x_{24}\right\}$

- $\left\{x_{25}<P\left(S_{2}, D_{2}, C_{1}, B_{1}\right)<x_{26}\right\}$

- $\left\{x_{27}<P\left(S_{2}, D_{2}, C_{1}, B_{2}\right)<x_{28}\right\}$

- $\left\{x_{29}<P\left(S_{2}, D_{2}, C_{2}, B_{1}\right)<x_{30}\right\}$

- $\left\{x_{31}<P\left(S_{2}, D_{2}, C_{2}, B_{2}\right)<x_{32}\right\}$ 
Para este exemplo, a evidência encontrada foi o estado $S_{1}$ da variável aleatória $S$. E a variável questionada após a evidência ocorrer é a variável $C$. Nesta situação, a inferência toma a forma abaixo Antonucci et al. (2014):

$$
\begin{aligned}
& K\left(X_{q} \mid X_{e}\right)=\frac{K\left(X_{q} \cap X_{e}\right)}{K\left(X_{e}\right)}=\frac{\sum_{X \backslash\left\{X_{q}, X_{e}\right\}}^{\otimes} K\left(X_{n}\right)}{\sum_{X \backslash\left\{X_{e}\right\}}^{\otimes} K\left(X_{n}\right)} \\
& K(C \mid S)=\frac{K(C \cap S)}{K(S)}=\frac{\sum_{\{S, D, C, B\} \backslash\{C, S\}}^{\otimes} K(S, D, C, B)}{\sum_{\{S, D, C, B\} \backslash\{S\}}^{\otimes} K(S, D, C, B)}
\end{aligned}
$$

O primeiro passo na inferência é descobrir as variáveis requisitadas $X_{r}$ dadas as evidências $X_{e}=$ $\{S\}$ e as variáveis consultadas $X_{q}=\{C\}$ através do algoritmo bayes ball. Este passo é necessário para que seja evitado desperdício computacional em variáveis irrelevantes para a inferência em questão.

Conforme Colla (2007) aplicou o algoritmo de Bayes Ball para encontrar somente as variáveis requistidas, este algoritmo também será aplicado nesta rede credal em específico:

$$
X_{r}=\{S, C, D\}
$$

\section{Grafo Moral}

E o grafo moral das variáveis requisitadas da rede credal segue conforme a figura abaixo:

Tabela 3.12: Grafo Moral da Rede Credal do apêndice A

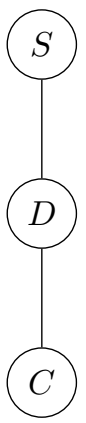




\section{Ordem de Elminação}

Através da heurística de Gibbs e Busca em Largura a árvore de dissecção gerou a seguinte ordem de eliminação

$$
[0,2,3]=[S, C, D]
$$

\section{Grafo Preenchidos}

E com a ordem de eliminação acima e o grafo moral, o seguinte grafo preenchido foi gerado através do algoritmo de fatoração simbólica:

Tabela 3.13: Grafo Preenchido da Rede Credal do apêndice A

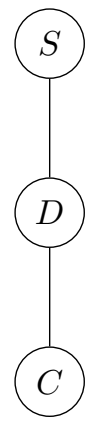

\section{Árvore de Elminação}

A árvore de elminação gerada para esta rede credal é:

Tabela 3.14: Árvore de eliminação do exemplo do apêndice A

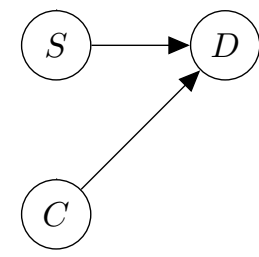


Esta árvore mostra quais operações numéricas podem ser executadas em paralelo durante a eliminação de variáveis. Neste exemplo em específico, as variáveis aleatórias $S, C$ podem ter seus cálculos feitos em paralelo. Estes cálculos númericos são as operações de marginalização e composião em conjuntos credais, conforme a figura abaixo mostra:

- Um primeiro processo faz $K(D, S)=K(D \mid S) \otimes K(S)$

- Um segundo processo faria $K(D, C)=K(C \mid D) \otimes K(D)$ se existisse a especificação do conjunto credal $K(D)$. Porém como este conjunto não foi especificado, este processo não necessita fazer nenhum cálculo númerico e mantém o conjunto credal $K(C \mid D)$

- E o último processo espera pelos dois primeiros processos para depois fazer o seguinte cálculo: $K(C, S)=\sum_{\{D, C, S\} \backslash\{C, S\}}^{\otimes} K(C \mid D) \otimes K(D, S)$

Estes passos são necessários para finalmente fazer a inferência:

$K\left(X_{q} \mid X_{e}\right)=\frac{K\left(X_{q} \cap X_{e}\right)}{K\left(X_{e}\right)}=K(C \mid S)=\frac{K(C \cap S)}{K(S)}=\frac{K\left(X_{q}, X_{e}\right)=\sum_{\{S, D, C, B\} \backslash\{C, S\}}^{\otimes} K(S, D, C, B)}{K\left(X_{e}\right)=\sum_{\{S, D, C, B\} \backslash\{S\}}^{\otimes} K(S, D, C, B)}$

Portanto a árvore de eliminação também é criada e executada para as variáveis de evidência $X_{e}=S$, para que o cálculo da inferência seja executado.

\subsection{Fatoração Numérica}

Com a fatoração simbólica, foi definido exclusivamente como a computação vai ser paralelizada. Porém precisa-se definir como vão ser feitos os cálculos para eliminar as variáveis ou conjunto credais. Neste nível de abstração, pode-se implementar tanto uma inferência exata quanto uma inferência aproximada, Augustin et al. (2014). Para este trabalho será implementada uma inferência aproximada utilizando o algoritmo $\mathrm{A} / \mathrm{R}+$, da Rocha et al. (2003).

Em uma rede credal, o algoritmo do cálculo numérico necessário para marginalizar um conjunto credal e executar a operação composição em conjuntos credais, que é análoga a operação de multiplicação de probabilidades, precisa saber resolver os seguintes problemas abaixos que serão enunciados nas seguintes seções:

1. Enumerar vértices de um conjunto credal;

2. Enumerar faces de vértices de um conjunto credal;

3. Acha o fecho convexo de vértices de um conjunto credal;

4. Programação linear;

5. Multiplicar, somar e dividir os vértices de um conjunto credal

As operações de multiplicação, soma e divisão dos vértices são operações algébricas com números reais e serão ilustrados na seção de inferência do exemplo de rede credal.

\subsubsection{Envoltória Convexa ou Fecho Convexo}

Dados dois pontos quaisquer $x_{1}$ e $x_{2}$ então o conjunto convexo $C \subset \mathbb{R}^{n}$ é definido Monticeli (2010) como:

$x_{1} \in C, x_{2} \in C \mid\left\{\Lambda . x_{1}+(1-\Lambda) . x 2\right\} \in C$, para todo $\Lambda \in[0,1]$

E dado um conjunto $D \subset \mathbb{R}^{n}$, o fecho convexo $C H$ é definido como o menor conjunto convexo em $\mathbb{R}^{n}$ que contenha $D$. Ou o fecho convexo pode ser definido como a intersecção de todos os conjuntos convexos em $\mathbb{R}^{n}$ que contenham $D$, Monticeli (2010), Cormen et al. (2009).

Para esta tese, como o algoritmo para achar o fecho convexo é auxiliar e necessário para a eliminação de variáveis em redes credais foi utilizada o algoritmo de Avis e Fukuda. (1992). Este algoritmo de envoltória convexa foi implementado por eles e disponibilizados com o código fonte no 
site Avis (2016). E no caso da aplicação da regra de bayes foi utilizado o algoritmo de envoltória convexa proposto por Fukuda e AlainProdon (1996) que possui a implementação disponível no site Fukuda (2016) com o seu código fonte.

\subsubsection{Enumeração de vértices e da faces}

Um semi-espaço é uma coleção de pontos $x$ tais que $\left\{x \cdot a^{T} \leq b\right.$, onde $a^{T}$ é um vetor não nulo pertencente a $\mathbb{R}^{n}$ e $b$ é um número escalar. E um poliedro $P$ é uma intersecção de um numero finito de semi-espaços $C_{i} \subset \mathbb{R}^{n}$, ou seja, $P=\bigcap_{i=1}^{p} C_{i}$.

O teorema de Minkowski-Weyl, Monticeli (2010), define um poliedro $P \subset \mathbb{R}^{n}$ com as seguintes afirmações equivalentes:

1. O poliedro $P$ pode ser definido como um sistema de inequações lineares, onde uma matriz real finita $A$ e algum vetor real finito $b$ tal que $P=\{x: A x \leq b\}$. A representação do poliedro desta maneira também pode ser definido como representação-H;

2. O poliedro $P$ pode ser definido como o fecho convexo de vetores reais $\left(x_{1}, x_{2}, \ldots, x_{r}\right) \subset \mathbb{R}^{n}$ adicionando os raios $\left(r_{1}, r_{2}, \ldots, r_{s}\right) \subset \mathbb{R}^{n}, P=C H\left(x_{1}, x_{2}, \ldots, x_{r}\right)+\left(r_{1}, r_{2}, \ldots, r_{s}\right)$. A representação do poliedro desta maneira também pode ser definido como representação-V.

A enumeração de vértices é o problema de trazer um poliedro da representação-H para a representação-V. E o contrário que é o problema de trazer um poliedro da representação- $V$ para a representação-H é chamado de enumeração das faces.

Para esta tese, como o algoritmo para enumerar os vértices e as faces são auxiliares e necessários também para a eliminação de variáveis em redes credais, foi utilizada o algoritmo de Avis e Fukuda. (1992). Estes algoritmos foram implementados por eles e disponibilizados com o código fonte no site Avis (2016). E no caso da aplicação da regra de bayes foi utilizado os algoritmos propostos por Fukuda e AlainProdon (1996) que possui a implementação disponível no site Fukuda (2016) com o seu código fonte.

\subsubsection{Programação Linear}

Em um poliedro $P$, e dada uma equação linear $a \cdot x^{T}=b$ pertencente a este poliedro $P$, onde $a$ é um vetor real e $b$ um escalar real, a programação linear acha os valores que maximizam ou minimizam a equação linear, Monticeli (2010).

Para esta tese, como o algoritmo de programação linear é auxiliar e necessário também para a eliminação de variáveis em redes credais, foi utilizada a implementação disponibilizada com o código fonte por Avis e Fukuda no site Avis (2016). E no caso da aplicação da regra de bayes foi utilizado a implementação feita por Fukuda disponível no site Fukuda (2016) com o seu código fonte.

\subsubsection{Inferências em Rede Credal - Continuação do exemplo}

Esta seção retoma o exemplo de rede credal utilizado na seção de fatoração simbólica, que representa o conjunto credal de probabilidades conjuntas $K(S, D, C, B)$. E de acordo com a árvore de eliminação, existem três processos de cálculos credais:

Um primeiro processo faz o cálculo credal do conjunto credal $K(D, S)$

$$
K(D, S)=K(D \mid S) \otimes K(S)
$$

1. Enumerar vértices de $K(S)$

2. Enumerar vértices de $K(D \mid S)$

3. Multiplicar vértices $K(D, S)=K(D \mid S) \otimes K(S)$

4. Achar o fecho convexo e então enumerar faces de $K(D, S)$ 
5. Programação Linear $K(D, S)$ para achar os novos limites do conjunto credal

Enumerar vértices de $K(S)$ resulta em:

- $\left\{x_{25}=0.25<P\left(S_{1}\right)<x_{26}=0.5\right\}$

- $\left\{x_{27}=0.5<P\left(S_{2}\right)<x_{28}=0.75\right\}$

$$
\text { Vértices do conjunto credal de } K(S)=\left[\begin{array}{cc}
\frac{1}{4} & \frac{3}{4} \\
\frac{1}{2} & \frac{1}{2}
\end{array}\right]
$$

Enumerar vértices de $K(D \mid S)$ resulta em:

- $\left\{x_{9}=0.2<P\left(D_{1} \mid S_{1}\right)<x_{10}=0.3\right\}$

- $\left\{x_{11}=0.3<P\left(D_{1} \mid S_{2}\right)<x_{12}=0.55\right\}$

- $\left\{x_{13}=0.15<P\left(D_{2} \mid S_{1}\right)<x_{14}=0.5\right\}$

- $\left\{x_{15}=0.0<P\left(D_{2} \mid S_{2}\right)<x_{16}=0.0\right\}$

$$
\text { Vértices do conjunto credal de } K(D \mid S)=\left[\begin{array}{cccc}
\frac{1}{5} & \frac{3}{10} & \frac{1}{2} & \frac{0}{1} \\
\frac{1}{5} & \frac{11}{20} & \frac{1}{4} & \frac{0}{1} \\
\frac{3}{10} & \frac{11}{20} & \frac{3}{20} & \frac{0}{1} \\
\frac{3}{10} & \frac{3}{10} & \frac{2}{5} & \frac{0}{1}
\end{array}\right]
$$

Vértices multiplicados do conjunto credal de $K(D, S)$ :

$$
\left[\begin{array}{llll}
\frac{1}{20} & \frac{9}{40} & \frac{1}{8} & \frac{0}{1} \\
\frac{1}{10} & \frac{3}{20} & \frac{1}{4} & \frac{0}{1} \\
\frac{1}{20} & \frac{33}{80} & \frac{1}{16} & \frac{0}{1} \\
\frac{1}{10} & \frac{11}{40} & \frac{1}{8} & \frac{0}{1} \\
\frac{3}{40} & \frac{33}{80} & \frac{3}{80} & \frac{0}{1} \\
\frac{3}{20} & \frac{11}{40} & \frac{3}{40} & \frac{0}{1} \\
\frac{3}{40} & \frac{9}{40} & \frac{1}{10} & \frac{0}{1} \\
\frac{3}{20} & \frac{3}{20} & \frac{1}{5} & \frac{0}{1}
\end{array}\right]
$$

Os vértices acima são o insumo para a operação de fecho convexo e enumeração de faces. E essas operações aplicadas nos vértices do conjunto $K(D, S)$ resultaram nas inequações:

$$
\left[\begin{array}{cccccc}
\frac{-1}{1} & +\frac{1}{1} \cdot K\left(D_{1}, S_{1}\right) & +\frac{1}{1} \cdot K\left(D_{1}, S_{2}\right) & +\frac{1}{1} \cdot K\left(D_{2}, S_{1}\right) & +\frac{1}{1} \cdot K\left(D_{2}, S_{2}\right) & \leq 0 \\
\frac{-33}{1} & +\frac{20}{1} \cdot K\left(D_{1}, S_{1}\right) & +\frac{60}{1} \cdot K\left(D_{1}, S_{2}\right) & +\frac{180}{1} \cdot K\left(D_{2}, S_{1}\right) & +\frac{0}{1} \cdot K\left(D_{2}, S_{2}\right) & \leq 0 \\
\frac{33}{1} & +\frac{-80}{1} \cdot K\left(D_{1}, S_{1}\right) & +\frac{-60}{1} \cdot K\left(D_{1}, S_{2}\right) & +\frac{-60}{1} \cdot K\left(D_{2}, S_{1}\right) & +\frac{0}{1} \cdot K\left(D_{2}, S_{2}\right) \leq 0 \\
\frac{-9}{1} & +\frac{-10}{1} \cdot K\left(D_{1}, S_{1}\right) & +\frac{30}{1} \cdot K\left(D_{1}, S_{2}\right) & +\frac{30}{1} \cdot K\left(D_{2}, S_{1}\right) & +\frac{0}{1} \cdot K\left(D_{2}, S_{2}\right) & \leq 0 \\
\frac{-21}{1} & +\frac{30}{1} \cdot K\left(D_{1}, S_{1}\right) & +\frac{70}{1} \cdot K\left(D_{1}, S_{2}\right) & +\frac{30}{1} \cdot K\left(D_{2}, S_{1}\right) & +\frac{0}{1} \cdot K\left(D_{2}, S_{2}\right) & \leq 0 \\
\frac{3}{1} & +\frac{60}{1} \cdot K\left(D_{1}, S_{1}\right) & +\frac{-10}{1} \cdot K\left(D_{1}, S_{2}\right) & +\frac{-30}{1} \cdot K\left(D_{2}, S_{1}\right) & +\frac{0}{1} \cdot K\left(D_{2}, S_{2}\right) & \leq 0 \\
\frac{51}{1} & +\frac{-105}{1} \cdot K\left(D_{1}, S_{1}\right) & +\frac{-95}{1} \cdot K\left(D_{1}, S_{2}\right) & +\frac{-105}{1} \cdot K\left(D_{2}, S_{1}\right) & +\frac{0}{1} \cdot K\left(D_{2}, S_{2}\right) \leq 0 \\
\frac{-3}{1} & +\frac{12}{1} \cdot K\left(D_{1}, S_{1}\right) & +\frac{4}{1} \cdot K\left(D_{1}, S_{2}\right) & +\frac{12}{1} \cdot K\left(D_{2}, S_{1}\right) & +\frac{0}{1} \cdot K\left(D_{2}, S_{2}\right) & \leq 0
\end{array}\right]
$$


Com as inequações acima, o próximo passo é a programação linear para achar os novos limites do conjunto credal $K(D, S)$ :

$$
\left[\begin{array}{l}
\frac{1}{20} \leq\left(D_{1}, S_{1}\right) \leq \frac{3}{20} \\
\frac{3}{20} \leq\left(D_{1}, S_{2}\right) \leq \frac{33}{80} \\
\frac{3}{80} \leq\left(D_{2}, S_{1}\right) \leq \frac{1}{4} \\
\frac{19}{40} \leq\left(D_{2}, S_{2}\right) \leq \frac{3}{5}
\end{array}\right]
$$

Um segundo processo em paralelo mantém o conjunto credal $K(C \mid D)$

- $\left\{x_{1}=0.15<P\left(C_{1} \mid D_{1}\right)<x_{2}=0.4\right\}$

- $\left\{x_{3}=0.05<P\left(C_{1} \mid D_{2}\right)<x_{4}=0.1\right\}$

- $\left\{x_{5}=0.5<P\left(C_{2} \mid D_{1}\right)<x_{6}=0.8\right\}$

- $\left\{x_{7}=0.0<P\left(C_{2} \mid D_{2}\right)<x_{8}=0.0\right\}$

E o último processo espera pelos dois primeiros processos para depois fazer o seguinte cálculo do conjunto credal $K(C, S)$

$K(C, S)=\sum_{\{D, C, S\} \backslash\{C, S\}}^{\otimes} K(C \mid D) \otimes K(D, S)$

1. Enumerar vértices de $K(C \mid D)$

2. Enumerar vértices de $K(D, S)$

3. Multiplicar vértices $K(D, C, S)=K(C \mid D) \otimes K(D, S)$

4. Achar o fecho convexo e então enumerar faces de $K(D, C, S)$

5. Programação Linear $K(D, C, S)$ para achar os novos limites do conjunto credal

6. Enumerar vértices de $K(D, C, S)$

7. Somar vértices $K(C, S)=\sum_{\{D, C, S\} \backslash\{C, S\}} K(D, C, S)$

8. Achar o fecho convexo e então enumerar faces de $K(C, S)$

9. Programação Linear $K(C, S)$ para achar os novos limites do conjunto credal

Vértices do conjunto credal de $K(C \mid D)=\left[\begin{array}{cccc}\frac{3}{20} & \frac{1}{20} & \frac{4}{5} & \frac{0}{1} \\ \frac{3}{20} & \frac{1}{10} & \frac{3}{4} & \frac{0}{1} \\ \frac{2}{5} & \frac{1}{10} & \frac{1}{2} & \frac{0}{1} \\ \frac{2}{5} & \frac{1}{20} & \frac{11}{20} & \frac{0}{1}\end{array}\right]$

Vértices do conjunto credal de $K(D, S)=\left[\begin{array}{cccc}\frac{1}{20} & \frac{3}{20} & \frac{1}{5} & \frac{3}{5} \\ \frac{3}{20} & \frac{3}{20} & \frac{1}{10} & \frac{3}{5} \\ \frac{3}{20} & \frac{17}{80} & \frac{3}{80} & \frac{3}{5} \\ \frac{1}{20} & \frac{5}{16} & \frac{3}{80} & \frac{3}{5} \\ \frac{1}{20} & \frac{33}{80} & \frac{3}{80} & \frac{1}{2} \\ \frac{1}{20} & \frac{3}{20} & \frac{1}{4} & \frac{11}{20} \\ \frac{1}{8} & \frac{3}{20} & \frac{1}{4} & \frac{19}{40} \\ \frac{3}{20} & \frac{3}{20} & \frac{9}{40} & \frac{19}{40} \\ \frac{3}{20} & \frac{27}{80} & \frac{3}{80} & \frac{19}{40} \\ \frac{1}{20} & \frac{9}{40} & \frac{1}{4} & \frac{19}{40} \\ \frac{1}{20} & \frac{33}{80} & \frac{1}{16} & \frac{19}{40} \\ \frac{3}{40} & \frac{33}{80} & \frac{3}{80} & \frac{19}{40}\end{array}\right]$ 
Após os vértices serem multiplicados, o fecho convexo foi aplicado e produziram os vértices abaixo:

$\left[\begin{array}{cccccccc}\frac{3}{400} & \frac{1}{25} & \frac{9}{400} & \frac{3}{25} & \frac{1}{100} & \frac{0}{1} & \frac{3}{100} & \frac{0}{1} \\ \frac{3}{400} & \frac{3}{80} & \frac{9}{400} & \frac{9}{80} & \frac{1}{50} & \frac{0}{1} & \frac{3}{50} & \frac{0}{1} \\ \frac{1}{50} & \frac{1}{40} & \frac{3}{50} & \frac{3}{40} & \frac{1}{50} & \frac{0}{1} & \frac{3}{50} & \frac{0}{1} \\ \frac{1}{50} & \frac{11}{400} & \frac{3}{50} & \frac{33}{400} & \frac{1}{100} & \frac{0}{1} & \frac{3}{100} & \frac{0}{1} \\ \frac{9}{400} & \frac{3}{25} & \frac{9}{400} & \frac{3}{25} & \frac{1}{200} & \frac{0}{1} & \frac{3}{100} & \frac{0}{1} \\ \frac{9}{400} & \frac{9}{80} & \frac{9}{400} & \frac{9}{80} & \frac{1}{100} & \frac{0}{1} & \frac{3}{50} & \frac{0}{1} \\ \frac{3}{50} & \frac{3}{40} & \frac{3}{50} & \frac{3}{40} & \frac{1}{100} & \frac{0}{1} & \frac{3}{50} & \frac{0}{1} \\ \frac{3}{50} & \frac{33}{400} & \frac{3}{50} & \frac{33}{400} & \frac{1}{200} & \frac{0}{1} & \frac{3}{100} & \frac{0}{1} \\ \frac{9}{400} & \frac{3}{25} & \frac{51}{1600} & \frac{17}{100} & \frac{3}{1600} & \frac{0}{1} & \frac{3}{100} & \frac{0}{1} \\ \frac{9}{400} & \frac{9}{80} & \frac{51}{1600} & \frac{51}{320} & \frac{3}{800} & \frac{0}{1} & \frac{3}{50} & \frac{0}{1} \\ \frac{3}{50} & \frac{3}{40} & \frac{17}{200} & \frac{17}{160} & \frac{3}{800} & \frac{0}{1} & \frac{3}{50} & \frac{0}{1} \\ \frac{3}{50} & \frac{33}{400} & \frac{17}{200} & \frac{187}{1600} & \frac{3}{1600} & \frac{0}{1} & \frac{3}{100} & \frac{0}{1} \\ \frac{3}{400} & \frac{1}{25} & \frac{3}{64} & \frac{1}{4} & \frac{3}{1600} & \frac{0}{1} & \frac{3}{100} & \frac{0}{1} \\ \frac{3}{400} & \frac{3}{80} & \frac{3}{64} & \frac{15}{64} & \frac{3}{800} & \frac{0}{1} & \frac{3}{50} & \frac{0}{1} \\ \frac{1}{50} & \frac{1}{40} & \frac{1}{8} & \frac{5}{32} & \frac{3}{800} & \frac{0}{1} & \frac{3}{50} & \frac{0}{1} \\ \frac{1}{50} & \frac{11}{400} & \frac{1}{8} & \frac{11}{64} & \frac{3}{1600} & \frac{0}{1} & \frac{3}{100} & \frac{0}{1} \\ \frac{3}{400} & \frac{1}{25} & \frac{99}{1600} & \frac{33}{100} & \frac{3}{1600} & \frac{0}{1} & \frac{1}{40} & \frac{0}{1} \\ \frac{3}{400} & \frac{3}{80} & \frac{99}{1600} & \frac{99}{320} & \frac{3}{800} & \frac{0}{1} & \frac{1}{20} & \frac{0}{1} \\ \frac{1}{50} & \frac{1}{40} & \frac{33}{200} & \frac{33}{160} & \frac{3}{800} & \frac{0}{1} & \frac{1}{20} & \frac{0}{1} \\ \frac{1}{50} & \frac{11}{400} & \frac{33}{200} & \frac{363}{1600} & \frac{3}{1600} & \frac{0}{1} & \frac{1}{40} & \frac{0}{1} \\ \frac{3}{400} & \frac{1}{25} & \frac{9}{400} & \frac{3}{25} & \frac{1}{80} & \frac{0}{1} & \frac{11}{400} & \frac{0}{1} \\ \frac{3}{400} & \frac{3}{80} & \frac{9}{400} & \frac{9}{80} & \frac{1}{40} & \frac{0}{1} & \frac{11}{200} & \frac{0}{1} \\ \frac{1}{50} & \frac{1}{40} & \frac{3}{50} & \frac{3}{40} & \frac{1}{40} & \frac{0}{1} & \frac{11}{200} & \frac{0}{1} \\ \frac{1}{50} & \frac{11}{400} & \frac{3}{50} & \frac{33}{400} & \frac{1}{80} & \frac{0}{1} & \frac{11}{400} & \frac{0}{1}\end{array}\right]$




$\left[\begin{array}{cccccccc}\frac{3}{160} & \frac{1}{10} & \frac{9}{400} & \frac{3}{25} & \frac{1}{80} & \frac{0}{1} & \frac{19}{800} & \frac{0}{1} \\ \frac{3}{160} & \frac{3}{32} & \frac{9}{400} & \frac{9}{80} & \frac{1}{40} & \frac{0}{1} & \frac{19}{400} & \frac{0}{1} \\ \frac{1}{20} & \frac{1}{16} & \frac{3}{50} & \frac{3}{40} & \frac{1}{40} & \frac{0}{1} & \frac{19}{400} & \frac{0}{1} \\ \frac{1}{20} & \frac{11}{160} & \frac{3}{50} & \frac{33}{400} & \frac{1}{80} & \frac{0}{1} & \frac{19}{800} & \frac{0}{1} \\ \frac{9}{400} & \frac{3}{25} & \frac{9}{400} & \frac{3}{25} & \frac{9}{800} & \frac{0}{1} & \frac{19}{800} & \frac{0}{1} \\ \frac{9}{400} & \frac{9}{80} & \frac{9}{400} & \frac{9}{80} & \frac{9}{400} & \frac{0}{1} & \frac{19}{400} & \frac{0}{1} \\ \frac{3}{50} & \frac{3}{40} & \frac{3}{50} & \frac{3}{40} & \frac{9}{400} & \frac{0}{1} & \frac{19}{400} & \frac{0}{1} \\ \frac{3}{50} & \frac{33}{400} & \frac{3}{50} & \frac{33}{400} & \frac{9}{800} & \frac{0}{1} & \frac{19}{800} & \frac{0}{1} \\ \frac{9}{400} & \frac{3}{25} & \frac{81}{1600} & \frac{27}{100} & \frac{3}{1600} & \frac{0}{1} & \frac{19}{800} & \frac{0}{1} \\ \frac{9}{400} & \frac{9}{80} & \frac{81}{1600} & \frac{81}{320} & \frac{3}{800} & \frac{0}{1} & \frac{19}{400} & \frac{0}{1} \\ \frac{3}{50} & \frac{3}{40} & \frac{27}{200} & \frac{27}{160} & \frac{3}{800} & \frac{0}{1} & \frac{19}{400} & \frac{0}{1} \\ \frac{3}{50} & \frac{33}{400} & \frac{27}{200} & \frac{297}{1600} & \frac{3}{1600} & \frac{0}{1} & \frac{19}{800} & \frac{0}{1} \\ \frac{3}{400} & \frac{1}{25} & \frac{27}{800} & \frac{9}{50} & \frac{1}{80} & \frac{0}{1} & \frac{19}{800} & \frac{0}{1} \\ \frac{3}{400} & \frac{3}{80} & \frac{27}{800} & \frac{27}{160} & \frac{1}{40} & \frac{0}{1} & \frac{19}{400} & \frac{0}{1} \\ \frac{1}{50} & \frac{1}{40} & \frac{9}{100} & \frac{9}{80} & \frac{1}{40} & \frac{0}{1} & \frac{19}{400} & \frac{0}{1} \\ \frac{1}{50} & \frac{11}{400} & \frac{9}{100} & \frac{99}{800} & \frac{1}{80} & \frac{0}{1} & \frac{19}{800} & \frac{0}{1} \\ \frac{3}{400} & \frac{1}{25} & \frac{99}{1600} & \frac{33}{100} & \frac{1}{320} & \frac{0}{1} & \frac{19}{800} & \frac{0}{1} \\ \frac{3}{400} & \frac{3}{80} & \frac{99}{1600} & \frac{99}{320} & \frac{1}{160} & \frac{0}{1} & \frac{19}{400} & \frac{0}{1} \\ \frac{1}{50} & \frac{1}{40} & \frac{33}{200} & \frac{33}{160} & \frac{1}{160} & \frac{0}{1} & \frac{19}{400} & \frac{0}{1} \\ \frac{1}{50} & \frac{11}{400} & \frac{33}{200} & \frac{363}{1600} & \frac{1}{320} & \frac{0}{1} & \frac{19}{800} & \frac{0}{1} \\ \frac{9}{800} & \frac{3}{50} & \frac{99}{1600} & \frac{33}{100} & \frac{3}{1600} & \frac{0}{1} & \frac{19}{800} & \frac{0}{1} \\ \frac{9}{800} & \frac{9}{160} & \frac{99}{1600} & \frac{99}{320} & \frac{3}{800} & \frac{0}{1} & \frac{19}{400} & \frac{0}{1} \\ \frac{3}{100} & \frac{3}{80} & \frac{33}{200} & \frac{33}{160} & \frac{3}{800} & \frac{0}{1} & \frac{19}{400} & \frac{0}{1} \\ \frac{3}{100} & \frac{33}{800} & \frac{33}{200} & \frac{363}{1600} & \frac{3}{1600} & \frac{0}{1} & \frac{19}{800} & \frac{0}{1}\end{array}\right]$

As inequações geradas do conjunto credal $K(D, C, S)$ podem ser expressos como $b+A x \leq 0$, onde $b$ é um vetor e $A$ é uma matriz de números reais. E $x$ é o vetor dos estados da probabilidade conjunta:

$$
\left[\begin{array}{l}
K\left(D_{1}, C_{1}, S_{1}\right) \\
K\left(D_{1}, C_{1}, S_{2}\right) \\
K\left(D_{1}, C_{2}, S_{1}\right) \\
K\left(D_{1}, C_{2}, S_{2}\right) \\
K\left(D_{2}, C_{1}, S_{1}\right) \\
K\left(D_{2}, C_{1}, S_{2}\right) \\
K\left(D_{2}, C_{2}, S_{1}\right) \\
K\left(D_{2}, C_{2}, S_{2}\right)
\end{array}\right]
$$


44 INFERÊNCIA EM REDES CREDAIS USANDO TÉCNICAS DE ÁLGEBRA LINEAR (FATORAÇÃO SIMBÓLICA)

$\mathrm{O}$ vetor $b$ das inequações geradas do conjunto credal $K(D, C, S)$ é :

$$
\left[\begin{array}{c}
\frac{-1}{1} \\
\frac{78351}{1} \\
\frac{89817}{1} \\
\frac{-551}{1} \\
\frac{-33}{1} \\
\frac{187929}{1} \\
\frac{182343}{1} \\
\frac{121429}{1} \\
\frac{43659}{1} \\
\frac{-4263}{1} \\
\frac{-16443}{1} \\
\frac{-10079}{1} \\
\frac{5883}{1} \\
\frac{-7049}{1} \\
\frac{-5149}{1} \\
\frac{92763}{1} \\
\frac{4797}{1} \\
\frac{1221}{1} \\
\frac{0}{1} \\
\frac{39}{1} \\
\frac{1425}{1} \\
\frac{-2736}{1} \\
\frac{-39}{1} \\
\frac{45309}{1} \\
\frac{-2109}{1} \\
\frac{2109}{1} \\
\frac{-927}{1} \\
\frac{-13}{1} \\
\frac{-23}{1} \\
\frac{-1}{1}
\end{array}\right]
$$




$$
\left[\begin{array}{c}
\frac{-168}{1} \\
\frac{741}{1} \\
\frac{-4767}{1} \\
\frac{-6711}{1} \\
\frac{-2553}{1} \\
\frac{-153}{1} \\
\frac{-1441}{1} \\
\frac{-3999}{1} \\
\frac{-117}{1} \\
\frac{-108}{1} \\
\frac{-57}{1} \\
\frac{-6}{1} \\
\frac{-33}{1} \\
\frac{-9111}{1} \\
\frac{-209}{1} \\
\frac{-1251}{1} \\
\frac{-1353}{1} \\
\frac{-8613}{1} \\
\frac{-399}{1} \\
\frac{7239}{1} \\
\frac{-567}{1} \\
\frac{405327}{1} \\
\frac{22347}{1} \\
\frac{140049}{1} \\
\frac{13311}{1} \\
\frac{5229}{1} \\
\frac{10143}{1} \\
\frac{-18221}{1} \\
\frac{156}{1} \\
\frac{-84}{1}
\end{array}\right]
$$


46 INFERÊNCIA EM REDES CREDAIS USANDO TÉCNICAS DE ÁLGEBRA LINEAR (FATORAÇÃO SIMBÓLICA)

$$
\left[\begin{array}{c}
\frac{567}{1} \\
\frac{-8451}{1} \\
\frac{-2871}{1} \\
\frac{-189}{1} \\
\frac{-81}{1} \\
\frac{-26241}{1} \\
\frac{20727}{1} \\
\frac{4263}{1} \\
\frac{835527}{1} \\
\frac{-16037}{1} \\
\frac{110481}{1} \\
\frac{193341}{1} \\
\frac{2817}{1} \\
\frac{3}{1} \\
\frac{-2874}{1} \\
\frac{-3}{1} \\
\frac{-93}{1} \\
\frac{-7311}{1} \\
\frac{0}{1} \\
\frac{-3}{1} \\
\frac{-42}{1} \\
\frac{-6}{1} \\
\frac{87}{1} \\
\frac{579}{1} \\
\frac{50859}{1} \\
\frac{213}{1} \\
\frac{1947}{1} \\
\frac{14637}{1} \\
\frac{109893}{1} \\
\frac{12789}{1} \\
\frac{279}{1}
\end{array}\right]
$$




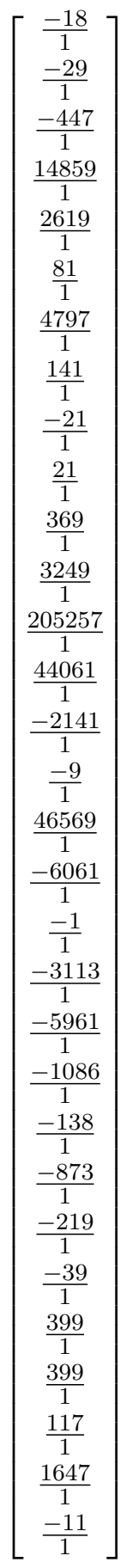


48 INFERÊNCIA EM REDES CREDAIS USANDO TÉCNICAS DE ÁLGEBRA LINEAR (FATORAÇÃO SIMBÓLICA) 


$$
\left[\begin{array}{c}
\frac{-1331}{1} \\
\frac{-83}{1} \\
\frac{-11}{1} \\
\frac{-1029}{1} \\
\frac{-77}{1} \\
\frac{-2899}{1} \\
\frac{-1257}{1} \\
\frac{627}{1} \\
\frac{30723}{1} \\
\frac{9801}{1} \\
\frac{50127}{1} \\
\frac{9147}{1} \\
\frac{1617}{1} \\
\frac{4560}{1} \\
\frac{47880}{1} \\
\frac{64239}{1} \\
\frac{46011}{1} \\
\frac{39}{1} \\
\frac{20349}{1} \\
\frac{20821}{1} \\
\frac{356763}{1} \\
\frac{21831}{1} \\
\frac{209}{1} \\
\frac{261}{1} \\
\frac{-657}{1} \\
\frac{-12}{1} \\
\frac{-9}{1} \\
\frac{-9033}{1} \\
\frac{-1551}{1} \\
\frac{-14811}{1}
\end{array}\right]
$$


50 INFERÊNCIA EM REDES CREDAIS USANDO TÉCNICAS DE ÁLGEBRA LINEAR (FATORAÇÃO SIMBÓLICA)

$$
\left[\begin{array}{c}
\frac{-519}{1} \\
\frac{-588}{1} \\
\frac{-804}{1} \\
\frac{-597}{1} \\
\frac{-6369}{1} \\
\frac{-9}{1} \\
\frac{-7733}{1} \\
\frac{-1581}{1} \\
\frac{-34533}{1} \\
\frac{-2451}{1} \\
\frac{-1563}{1} \\
\frac{-1281}{1} \\
\frac{-32847}{1} \\
\frac{-4881}{1} \\
\frac{-2523}{1} \\
\frac{51}{1} \\
\frac{-117}{1} \\
\frac{-969}{1} \\
\frac{798}{1} \\
\frac{1113}{1} \\
\frac{24021}{1} \\
\frac{51}{1} \\
\frac{33}{1} \\
\frac{7137}{1} \\
\frac{13923}{1} \\
\frac{2409}{1} \\
\frac{8463}{1} \\
\frac{2907}{1} \\
\frac{153}{1} \\
\frac{9}{1}
\end{array}\right]
$$




$\left[\begin{array}{c}\frac{33}{1} \\ \frac{33}{1} \\ \frac{99}{1} \\ \frac{42}{1} \\ \frac{171}{1} \\ \frac{285}{1} \\ \frac{5643}{1} \\ \frac{0}{1} \\ \frac{78351}{1} \\ \frac{3381}{1} \\ \frac{128673}{1} \\ \frac{3867}{1} \\ \frac{77019}{1} \\ \frac{1581}{1} \\ \frac{-84249}{1} \\ \frac{-10469}{1} \\ \frac{-9112}{1} \\ \frac{-17139}{1} \\ \frac{-87}{1} \\ \frac{-453}{1} \\ \frac{3}{1} \\ \frac{33}{1} \\ \frac{87}{1} \\ \frac{129909}{1} \\ \frac{5583}{1} \\ \frac{87}{1} \\ \frac{7221}{1} \\ \frac{3219}{1} \\ \frac{48723}{1} \\ \frac{111}{1}\end{array}\right]$


52 INFERÊNCIA EM REDES CREDAIS USANDO TÉCNICAS DE ÁLGEBRA LINEAR (FATORAÇÃO SIMBÓLICA)

$$
\left[\begin{array}{c}
\frac{204}{1} \\
\frac{12}{1} \\
\frac{87}{1} \\
\frac{-3}{1} \\
\frac{-51}{1} \\
\frac{141}{1} \\
\frac{213}{1} \\
\frac{33}{1} \\
\frac{831}{1} \\
\frac{-9}{1} \\
\frac{-2346}{1} \\
\frac{-1059}{1} \\
\frac{26676}{1} \\
\frac{15903}{1} \\
\frac{22401}{1} \\
\frac{4959}{1} \\
\frac{177493}{1} \\
\frac{561}{1} \\
\frac{7797}{1} \\
\frac{14787}{1} \\
\frac{4959}{1} \\
\frac{47589}{1} \\
\frac{-489}{1} \\
\frac{-3}{1} \\
\frac{4071}{1} \\
\frac{-1653}{1} \\
\frac{-2001}{1} \\
\frac{-451}{1} \\
\frac{-36}{1} \\
\frac{8987}{1}
\end{array}\right]
$$




$\left[\begin{array}{c}\frac{12597}{1} \\ \frac{-33}{1} \\ \frac{21}{1} \\ \frac{5007}{1} \\ \frac{921}{1} \\ \frac{7821}{1} \\ \frac{5829}{1} \\ \frac{0}{1} \\ \frac{47101}{1} \\ \frac{158175}{1} \\ \frac{2211}{1} \\ \frac{16443}{1} \\ \frac{7092}{1} \\ \frac{2414843}{1} \\ \frac{19}{1} \\ \frac{2907}{1} \\ \frac{34599}{1} \\ \frac{417525}{1} \\ \frac{212553}{1} \\ \frac{168891}{1} \\ \frac{194689}{1} \\ \frac{9424665}{1} \\ \frac{-417}{1} \\ \frac{-3091}{1} \\ \frac{-7207}{1} \\ \frac{-61061}{1} \\ \frac{-209}{1} \\ \frac{-7381}{1} \\ \frac{-93}{1} \\ \frac{201}{1}\end{array}\right]$


54 INFERÊNCIA EM REDES CREDAIS USANDO TÉCNICAS DE ÁLGEBRA LINEAR (FATORAÇÃO SIMBÓLICA)

$\left[\begin{array}{c}\frac{-100097}{1} \\ \frac{-1069}{1} \\ \frac{-291}{1} \\ \frac{-4179}{1} \\ \frac{-352}{1} \\ \frac{-1273}{1} \\ \frac{-16321}{1} \\ \frac{-35221}{1} \\ \frac{-261}{1} \\ \frac{-196}{1} \\ \frac{-972}{1} \\ \frac{-4917}{1} \\ \frac{-5289}{1} \\ \frac{-36}{1} \\ \frac{-1311}{1} \\ \frac{-19}{1} \\ \frac{-817}{1} \\ \frac{2109}{1} \\ \frac{0}{1} \\ \frac{5463}{1} \\ \frac{231}{1} \\ \frac{249}{1} \\ \frac{21}{1} \\ \frac{216}{1} \\ \frac{39}{1}\end{array}\right]$


E a matriz $A$ das inequações geradas do conjunto credal $K(D, C, S)$ é:

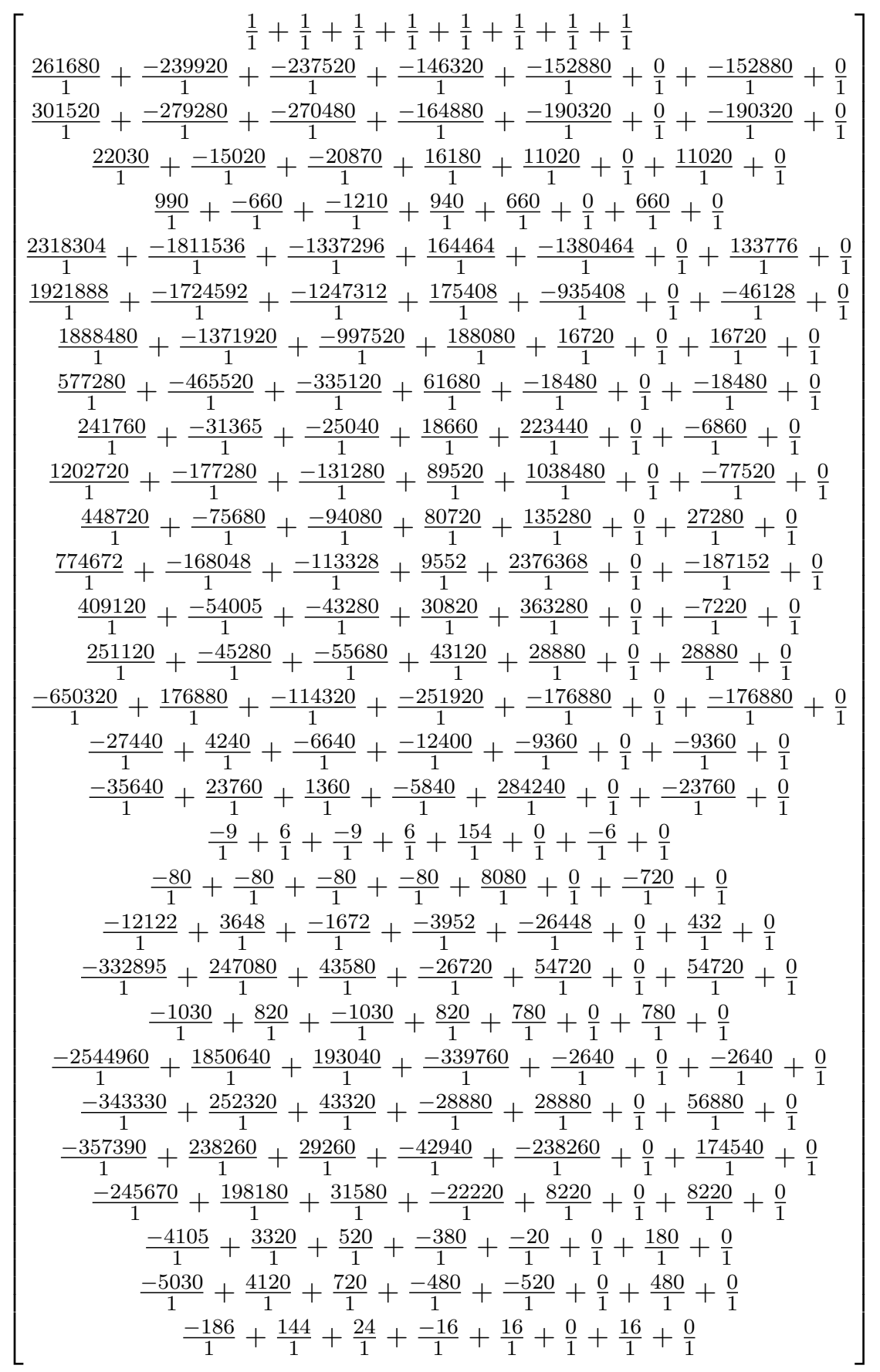




$$
\begin{aligned}
& \frac{-53305}{1}+\frac{43220}{1}+\frac{6820}{1}+\frac{-4880}{1}+\frac{2880}{1}+\frac{0}{1}+\frac{880}{1}+\frac{0}{1} \\
& \frac{-1520}{1}+\frac{-1520}{1}+\frac{-1520}{1}+\frac{-1520}{1}+\frac{-13680}{1}+\frac{0}{1}+\frac{-480}{1}+\frac{0}{1} \\
& \frac{-234585}{1}+\frac{96465}{1}+\frac{204415}{1}+\frac{-23385}{1}+\frac{-456015}{1}+\frac{0}{1}+\frac{239085}{1}+\frac{0}{1} \\
& \frac{-262560}{1}+\frac{204240}{1}+\frac{141440}{1}+\frac{-24960}{1}+\frac{-29040}{1}+\frac{0}{1}+\frac{24960}{1}+\frac{0}{1} \\
& \frac{-254590}{1}+\frac{65610}{1}+\frac{252410}{1}+\frac{-35790}{1}+\frac{-369810}{1}+\frac{0}{1}+\frac{184590}{1}+\frac{0}{1} \\
& \frac{-76330}{1}+\frac{17070}{1}+\frac{76670}{1}+\frac{-13530}{1}+\frac{-34470}{1}+\frac{0}{1}+\frac{13530}{1}+\frac{0}{1} \\
& \frac{-58912}{1}+\frac{18848}{1}+\frac{59888}{1}+\frac{-4912}{1}+\frac{-192688}{1}+\frac{0}{1}+\frac{101072}{1}+\frac{0}{1} \\
& \frac{-203490}{1}+\frac{62910}{1}+\frac{203510}{1}+\frac{-18490}{1}+\frac{-499410}{1}+\frac{0}{1}+\frac{250590}{1}+\frac{0}{1} \\
& \frac{-5370}{1}+\frac{1830}{1}+\frac{5630}{1}+\frac{-370}{1}+\frac{-12330}{1}+\frac{0}{1}+\frac{5670}{1}+\frac{0}{1} \\
& \frac{15}{1}+\frac{15}{1}+\frac{715}{1}+\frac{715}{1}+\frac{135}{1}+\frac{0}{1}+\frac{135}{1}+\frac{0}{1} \\
& \frac{0}{1}+\frac{0}{1}+\frac{380}{1}+\frac{380}{1}+\frac{0}{1}+\frac{0}{1}+\frac{120}{1}+\frac{0}{1} \\
& \frac{-5195}{1}+\frac{1005}{1}+\frac{5205}{1}+\frac{-945}{1}+\frac{-1005}{1}+\frac{0}{1}+\frac{495}{1}+\frac{0}{1} \\
& \frac{-140560}{1}+\frac{26640}{1}+\frac{141040}{1}+\frac{-26160}{1}+\frac{-11040}{1}+\frac{0}{1}+\frac{4560}{1}+\frac{0}{1} \\
& \frac{-321120}{1}+\frac{214080}{1}+\frac{190280}{1}+\frac{-25620}{1}+\frac{-214080}{1}+\frac{0}{1}+\frac{160920}{1}+\frac{0}{1} \\
& \frac{-6152}{1}+\frac{2056}{1}+\frac{6520}{1}+\frac{-320}{1}+\frac{-26600}{1}+\frac{0}{1}+\frac{14440}{1}+\frac{0}{1} \\
& \frac{-360}{1}+\frac{240}{1}+\frac{8640}{1}+\frac{8140}{1}+\frac{-240}{1}+\frac{0}{1}+\frac{2760}{1}+\frac{0}{1} \\
& \frac{120}{1}+\frac{120}{1}+\frac{8920}{1}+\frac{8920}{1}+\frac{1080}{1}+\frac{0}{1}+\frac{2280}{1}+\frac{0}{1} \\
& \frac{-285960}{1}+\frac{215640}{1}+\frac{145240}{1}+\frac{-21960}{1}+\frac{-65640}{1}+\frac{0}{1}+\frac{84360}{1}+\frac{0}{1} \\
& \frac{30}{1}+\frac{30}{1}+\frac{2630}{1}+\frac{2630}{1}+\frac{570}{1}+\frac{0}{1}+\frac{570}{1}+\frac{0}{1} \\
& \frac{102980}{1}+\frac{-104120}{1}+\frac{-106020}{1}+\frac{47880}{1}+\frac{-108680}{1}+\frac{0}{1}+\frac{33720}{1}+\frac{0}{1} \\
& \frac{32860}{1}+\frac{-23240}{1}+\frac{-30940}{1}+\frac{23160}{1}+\frac{15240}{1}+\frac{0}{1}+\frac{15240}{1}+\frac{0}{1} \\
& \frac{2900160}{1}+\frac{-3597840}{1}+\frac{-2533840}{1}+\frac{354160}{1}+\frac{-1934960}{1}+\frac{0}{1}+\frac{-107760}{1}+\frac{0}{1} \\
& \frac{150560}{1}+\frac{-191440}{1}+\frac{-135440}{1}+\frac{16560}{1}+\frac{1589840}{1}+\frac{0}{1}+\frac{-141360}{1}+\frac{0}{1} \\
& \frac{1166080}{1}+\frac{-1364720}{1}+\frac{-950320}{1}+\frac{174480}{1}+\frac{-59280}{1}+\frac{0}{1}+\frac{-59280}{1}+\frac{0}{1} \\
& \frac{172040}{1}+\frac{-176560}{1}+\frac{-146960}{1}+\frac{55440}{1}+\frac{2722160}{1}+\frac{0}{1}+\frac{-194640}{1}+\frac{0}{1} \\
& \frac{255680}{1}+\frac{-211120}{1}+\frac{-188720}{1}+\frac{112080}{1}+\frac{1227120}{1}+\frac{0}{1}+\frac{-32880}{1}+\frac{0}{1} \\
& \frac{97688}{1}+\frac{-91792}{1}+\frac{-66512}{1}+\frac{8208}{1}+\frac{770672}{1}+\frac{0}{1}+\frac{-68208}{1}+\frac{0}{1} \\
& \frac{2901280}{1}+\frac{-2137520}{1}+\frac{-2075120}{1}+\frac{1481680}{1}+\frac{17481520}{1}+\frac{0}{1}+\frac{-302480}{1}+\frac{0}{1} \\
& \frac{1731}{1}+\frac{-1404}{1}+\frac{-1044}{1}+\frac{96}{1}+\frac{20464}{1}+\frac{0}{1}+\frac{-1596}{1}+\frac{0}{1} \\
& \frac{3995}{1}+\frac{-2830}{1}+\frac{-3855}{1}+\frac{2970}{1}+\frac{1830}{1}+\frac{0}{1}+\frac{1830}{1}+\frac{0}{1}
\end{aligned}
$$




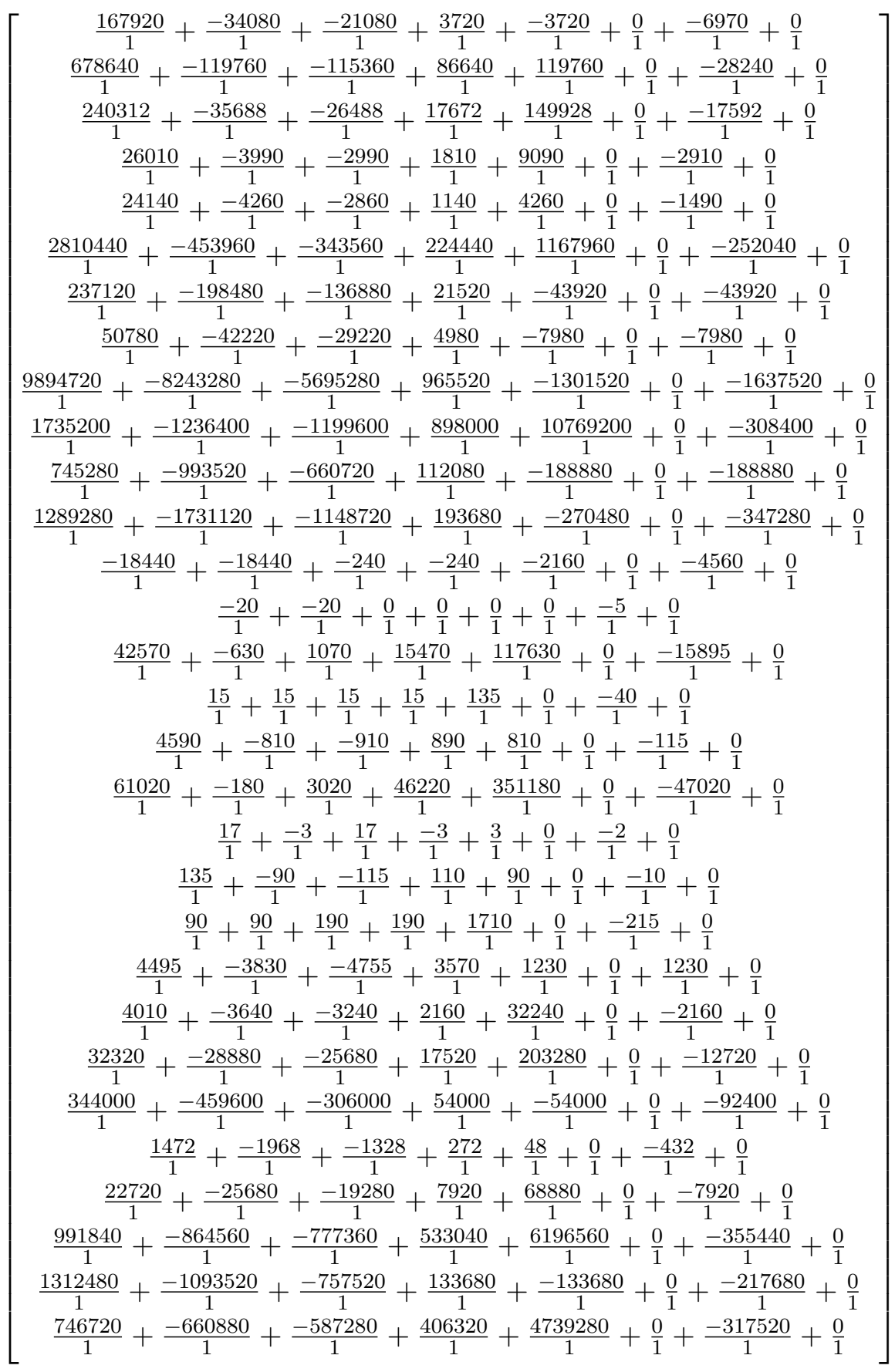




$$
\begin{aligned}
& \frac{6400}{1}+\frac{-5840}{1}+\frac{-5200}{1}+\frac{3440}{1}+\frac{18000}{1}+\frac{0}{1}+\frac{-3600}{1}+\frac{0}{1} \\
& \frac{2885}{1}+\frac{-2290}{1}+\frac{-2865}{1}+\frac{2310}{1}+\frac{2490}{1}+\frac{0}{1}+\frac{90}{1}+\frac{0}{1} \\
& \frac{-610}{1}+\frac{540}{1}+\frac{-610}{1}+\frac{540}{1}+\frac{1060}{1}+\frac{0}{1}+\frac{260}{1}+\frac{0}{1} \\
& \frac{13550}{1}+\frac{-8300}{1}+\frac{-11750}{1}+\frac{10100}{1}+\frac{17100}{1}+\frac{0}{1}+\frac{3500}{1}+\frac{0}{1} \\
& \frac{-93280}{1}+\frac{-100080}{1}+\frac{-4080}{1}+\frac{720}{1}+\frac{-720}{1}+\frac{0}{1}+\frac{-24720}{1}+\frac{0}{1} \\
& \frac{-12480}{1}+\frac{-19280}{1}+\frac{-3280}{1}+\frac{1520}{1}+\frac{6480}{1}+\frac{0}{1}+\frac{-5520}{1}+\frac{0}{1} \\
& \frac{480}{1}+\frac{-1220}{1}+\frac{-720}{1}+\frac{480}{1}+\frac{5520}{1}+\frac{0}{1}+\frac{-480}{1}+\frac{0}{1} \\
& \frac{29760}{1}+\frac{-41520}{1}+\frac{-27440}{1}+\frac{4240}{1}+\frac{-9360}{1}+\frac{0}{1}+\frac{-9360}{1}+\frac{0}{1} \\
& \frac{1040}{1}+\frac{-1360}{1}+\frac{-960}{1}+\frac{240}{1}+\frac{-240}{1}+\frac{0}{1}+\frac{-240}{1}+\frac{0}{1} \\
& \frac{45}{1}+\frac{-30}{1}+\frac{95}{1}+\frac{170}{1}+\frac{30}{1}+\frac{0}{1}+\frac{30}{1}+\frac{0}{1} \\
& \frac{-95}{1}+\frac{-170}{1}+\frac{-45}{1}+\frac{30}{1}+\frac{-30}{1}+\frac{0}{1}+\frac{-30}{1}+\frac{0}{1} \\
& \frac{-2280}{1}+\frac{-2280}{1}+\frac{-80}{1}+\frac{-80}{1}+\frac{-720}{1}+\frac{0}{1}+\frac{-720}{1}+\frac{0}{1} \\
& \frac{74005}{1}+\frac{-68970}{1}+\frac{-82745}{1}+\frac{45030}{1}+\frac{-105830}{1}+\frac{0}{1}+\frac{42570}{1}+\frac{0}{1} \\
& \frac{166060}{1}+\frac{-1133540}{1}+\frac{-920740}{1}+\frac{-343140}{1}+\frac{-8252460}{1}+\frac{0}{1}+\frac{3442740}{1}+\frac{0}{1} \\
& \frac{104120}{1}+\frac{-306280}{1}+\frac{-313880}{1}+\frac{-2280}{1}+\frac{-2140920}{1}+\frac{0}{1}+\frac{916680}{1}+\frac{0}{1} \\
& \frac{187180}{1}+\frac{-129920}{1}+\frac{-178020}{1}+\frac{135680}{1}+\frac{-33680}{1}+\frac{0}{1}+\frac{68320}{1}+\frac{0}{1} \\
& \frac{777}{1}+\frac{-558}{1}+\frac{-733}{1}+\frac{602}{1}+\frac{78}{1}+\frac{0}{1}+\frac{78}{1}+\frac{0}{1} \\
& \frac{82840}{1}+\frac{-327560}{1}+\frac{-335160}{1}+\frac{6840}{1}+\frac{-2332440}{1}+\frac{0}{1}+\frac{1003560}{1}+\frac{0}{1} \\
& \frac{138680}{1}+\frac{-66520}{1}+\frac{-79120}{1}+\frac{91880}{1}+\frac{-648280}{1}+\frac{0}{1}+\frac{377720}{1}+\frac{0}{1} \\
& \frac{5}{1}+\frac{5}{1}+\frac{5}{1}+\frac{5}{1}+\frac{-95}{1}+\frac{0}{1}+\frac{45}{1}+\frac{0}{1} \\
& \frac{65320}{1}+\frac{-31880}{1}+\frac{-33680}{1}+\frac{47320}{1}+\frac{-314120}{1}+\frac{0}{1}+\frac{171880}{1}+\frac{0}{1} \\
& \frac{2040}{1}+\frac{-360}{1}+\frac{38040}{1}+\frac{40040}{1}+\frac{360}{1}+\frac{0}{1}+\frac{12360}{1}+\frac{0}{1} \\
& \frac{30165}{1}+\frac{-15735}{1}+\frac{-16585}{1}+\frac{21665}{1}+\frac{-82515}{1}+\frac{0}{1}+\frac{41985}{1}+\frac{0}{1} \\
& \frac{795}{1}+\frac{-405}{1}+\frac{295}{1}+\frac{1295}{1}+\frac{-1845}{1}+\frac{0}{1}+\frac{1155}{1}+\frac{0}{1} \\
& \frac{27970}{1}+\frac{-15230}{1}+\frac{-16030}{1}+\frac{19970}{1}+\frac{-72270}{1}+\frac{0}{1}+\frac{35730}{1}+\frac{0}{1} \\
& \frac{10035}{1}+\frac{-6690}{1}+\frac{-8215}{1}+\frac{7910}{1}+\frac{-11310}{1}+\frac{0}{1}+\frac{6690}{1}+\frac{0}{1} \\
& \frac{4485}{1}+\frac{-3390}{1}+\frac{-4265}{1}+\frac{3610}{1}+\frac{-1410}{1}+\frac{0}{1}+\frac{990}{1}+\frac{0}{1} \\
& \frac{-1805}{1}+\frac{-1805}{1}+\frac{-855}{1}+\frac{-855}{1}+\frac{-16245}{1}+\frac{0}{1}+\frac{6855}{1}+\frac{0}{1} \\
& \frac{-2630}{1}+\frac{-2630}{1}+\frac{-30}{1}+\frac{-30}{1}+\frac{-570}{1}+\frac{0}{1}+\frac{-570}{1}+\frac{0}{1} \\
& \frac{-1390}{1}+\frac{-540}{1}+\frac{460}{1}+\frac{-140}{1}+\frac{1140}{1}+\frac{0}{1}+\frac{-360}{1}+\frac{0}{1}
\end{aligned}
$$




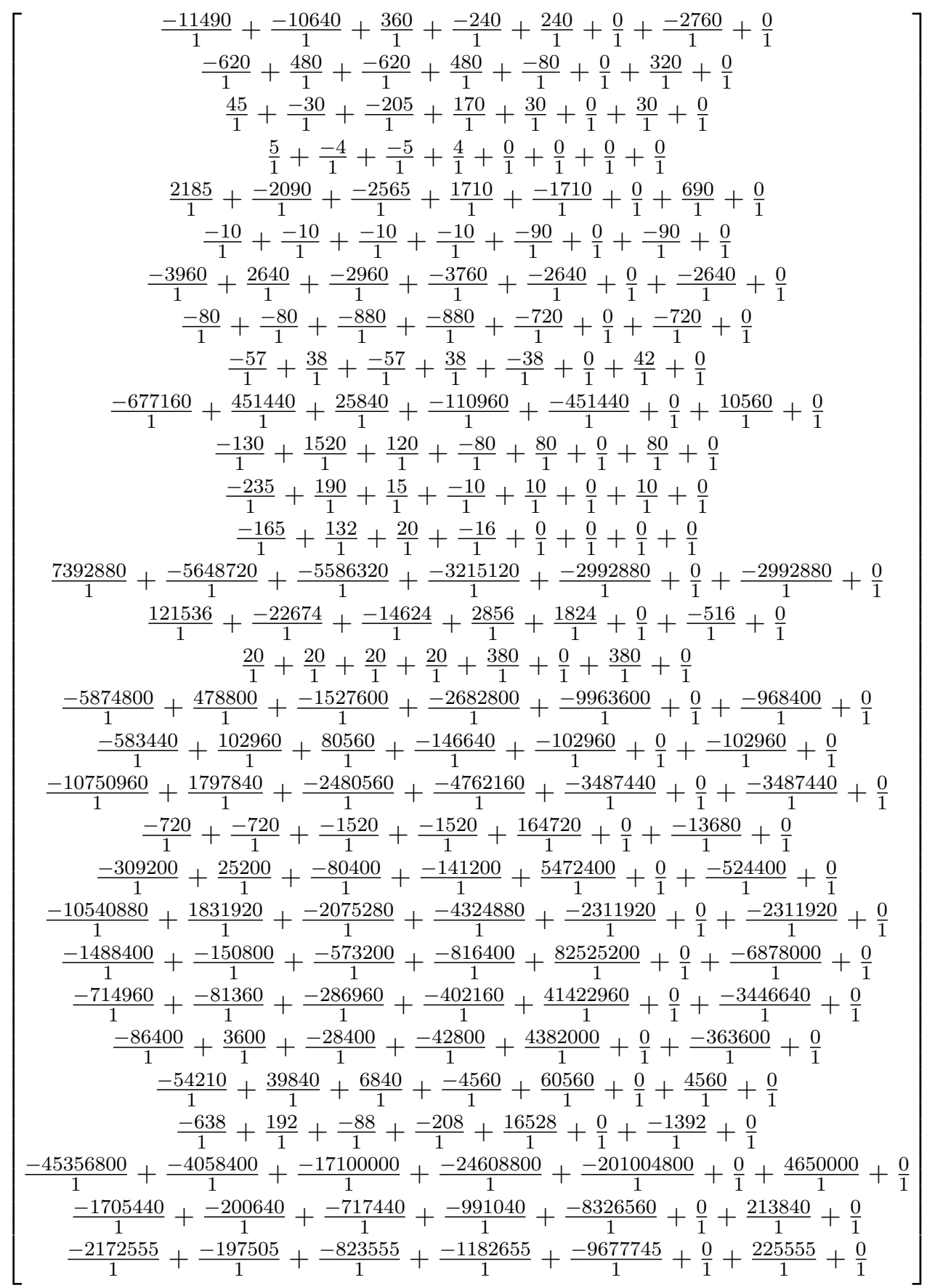




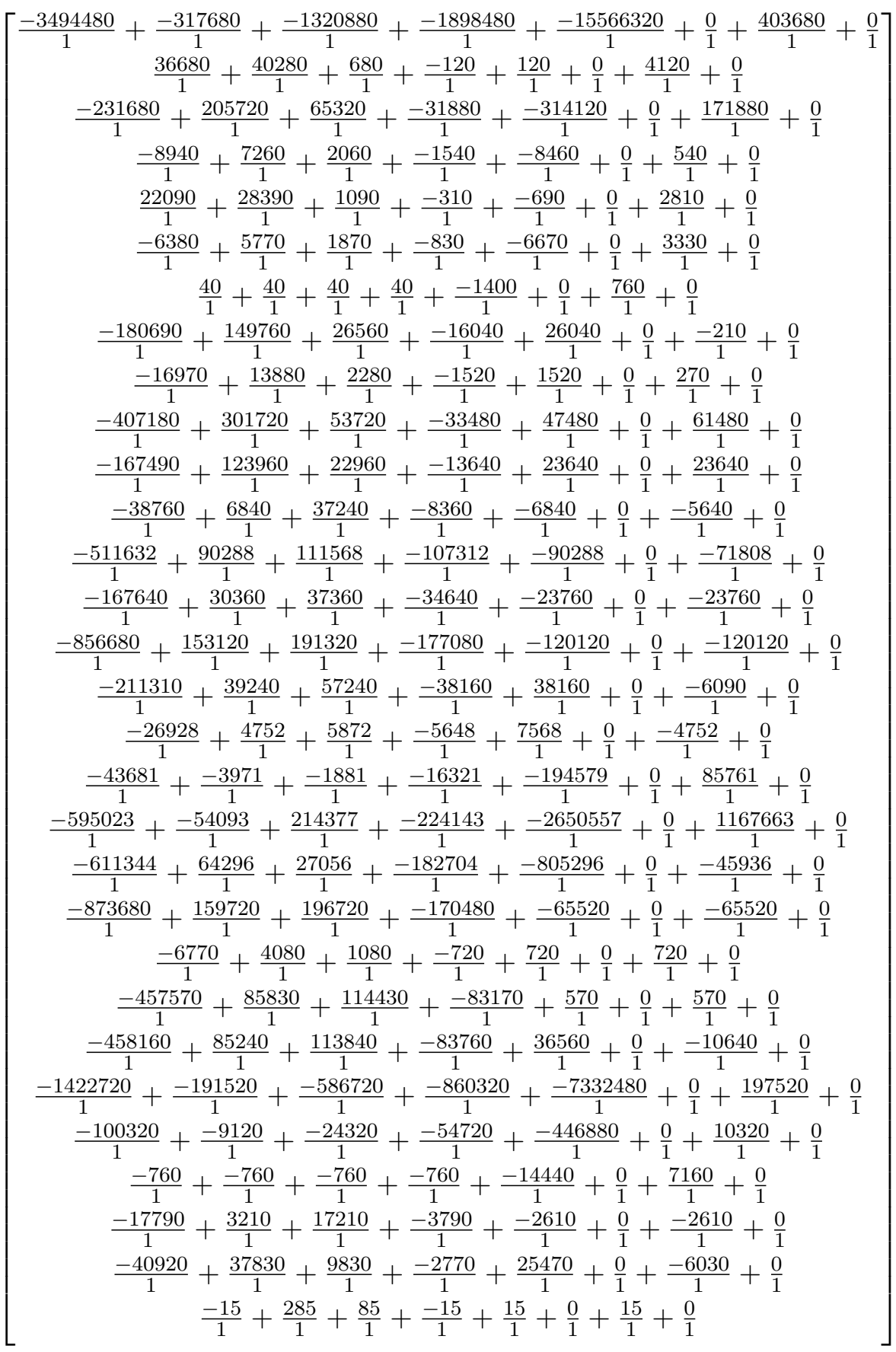




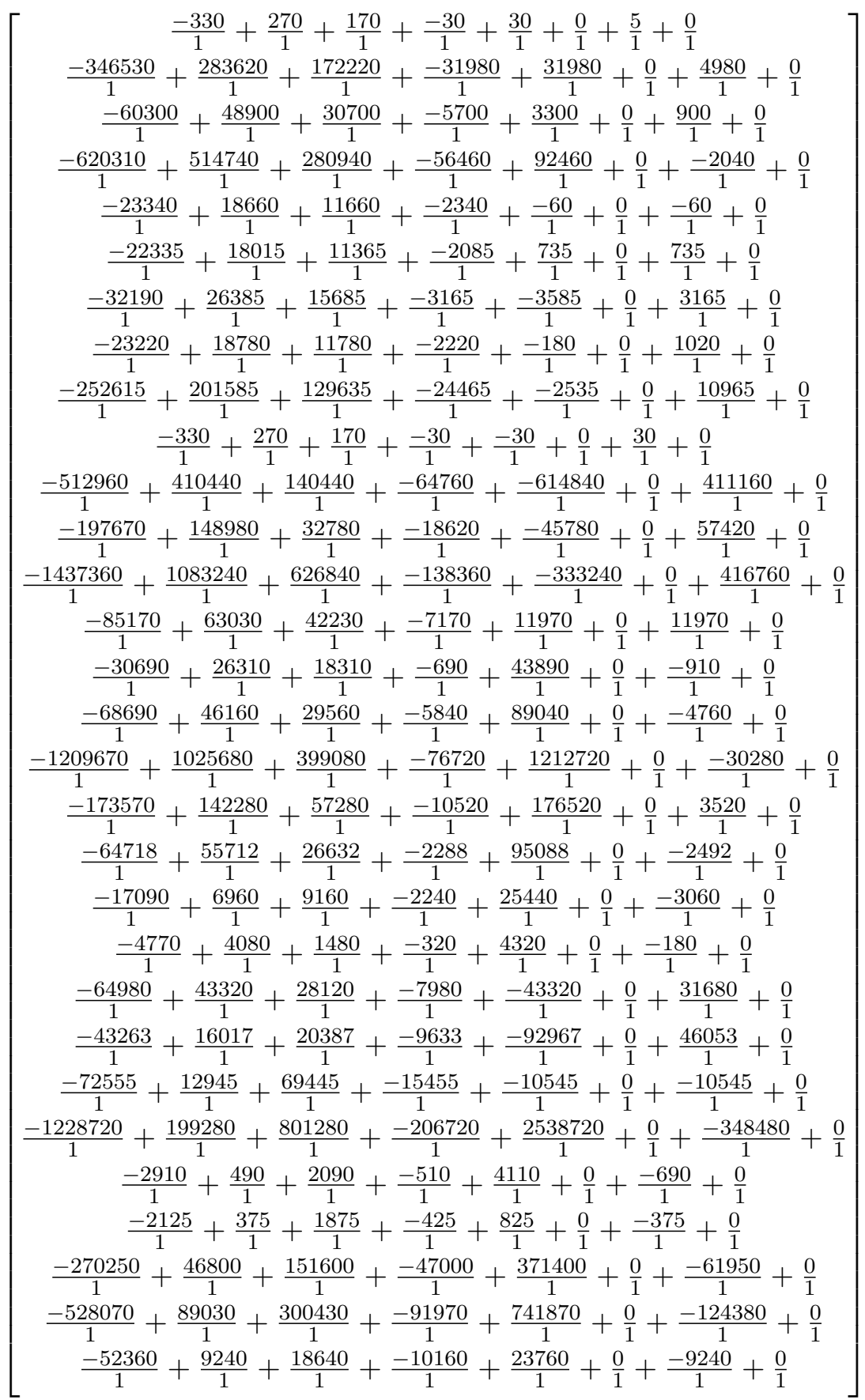




$$
\begin{aligned}
& \frac{-115920}{1}+\frac{2880}{1}+\frac{27080}{1}+\frac{-25720}{1}+\frac{-20280}{1}+\frac{0}{1}+\frac{-20280}{1}+\frac{0}{1} \\
& \frac{-98710}{1}+\frac{13490}{1}+\frac{56290}{1}+\frac{-17510}{1}+\frac{137610}{1}+\frac{0}{1}+\frac{-23940}{1}+\frac{0}{1} \\
& \frac{-7030}{1}+\frac{1130}{1}+\frac{4570}{1}+\frac{-1190}{1}+\frac{12330}{1}+\frac{0}{1}+\frac{-2070}{1}+\frac{0}{1} \\
& \frac{-1020}{1}+\frac{180}{1}+\frac{5380}{1}+\frac{-1020}{1}+\frac{-180}{1}+\frac{0}{1}+\frac{-180}{1}+\frac{0}{1} \\
& \frac{5830}{1}+\frac{-1170}{1}+\frac{5830}{1}+\frac{-1170}{1}+\frac{-1230}{1}+\frac{0}{1}+\frac{-30}{1}+\frac{0}{1} \\
& \frac{-44950}{1}+\frac{8850}{1}+\frac{45250}{1}+\frac{-8550}{1}+\frac{-1050}{1}+\frac{0}{1}+\frac{-1050}{1}+\frac{0}{1} \\
& \frac{-33110}{1}+\frac{6690}{1}+\frac{32890}{1}+\frac{-6510}{1}+\frac{-5490}{1}+\frac{0}{1}+\frac{510}{1}+\frac{0}{1} \\
& \frac{-2969}{1}+\frac{531}{1}+\frac{2871}{1}+\frac{-629}{1}+\frac{-411}{1}+\frac{0}{1}+\frac{-411}{1}+\frac{0}{1} \\
& \frac{-61370}{1}+\frac{10830}{1}+\frac{60230}{1}+\frac{-11970}{1}+\frac{-10830}{1}+\frac{0}{1}+\frac{4770}{1}+\frac{0}{1} \\
& \frac{-15428}{1}+\frac{2052}{1}+\frac{13072}{1}+\frac{-3648}{1}+\frac{-19152}{1}+\frac{0}{1}+\frac{8568}{1}+\frac{0}{1} \\
& \frac{-98002}{1}+\frac{-1482}{1}+\frac{53998}{1}+\frac{-31882}{1}+\frac{-317718}{1}+\frac{0}{1}+\frac{139962}{1}+\frac{0}{1} \\
& \frac{-16}{1}+\frac{3}{1}+\frac{16}{1}+\frac{-3}{1}+\frac{0}{1}+\frac{0}{1}+\frac{0}{1}+\frac{0}{1} \\
& \frac{-1111120}{1}+\frac{17480}{1}+\frac{261680}{1}+\frac{-239920}{1}+\frac{-152880}{1}+\frac{0}{1}+\frac{-152880}{1}+\frac{0}{1} \\
& \frac{-32176}{1}+\frac{3384}{1}+\frac{1424}{1}+\frac{-9616}{1}+\frac{463856}{1}+\frac{0}{1}+\frac{-42384}{1}+\frac{0}{1} \\
& \frac{-8194560}{1}+\frac{1393440}{1}+\frac{5435440}{1}+\frac{-1332560}{1}+\frac{20102160}{1}+\frac{0}{1}+\frac{-2288640}{1}+\frac{0}{1} \\
& \frac{-254350}{1}+\frac{44250}{1}+\frac{190650}{1}+\frac{-44750}{1}+\frac{329550}{1}+\frac{0}{1}+\frac{-55050}{1}+\frac{0}{1} \\
& \frac{-4131824}{1}+\frac{702816}{1}+\frac{2680976}{1}+\frac{-689584}{1}+\frac{8690544}{1}+\frac{0}{1}+\frac{-1150416}{1}+\frac{0}{1} \\
& \frac{-93634}{1}+\frac{16206}{1}+\frac{67566}{1}+\frac{-16394}{1}+\frac{129354}{1}+\frac{0}{1}+\frac{-21606}{1}+\frac{0}{1} \\
& \frac{-2995980}{1}+\frac{2831520}{1}+\frac{759520}{1}+\frac{-172880}{1}+\frac{3971280}{1}+\frac{0}{1}+\frac{-157320}{1}+\frac{0}{1} \\
& \frac{-298005}{1}+\frac{260120}{1}+\frac{76820}{1}+\frac{-12480}{1}+\frac{477280}{1}+\frac{0}{1}+\frac{30780}{1}+\frac{0}{1} \\
& \frac{-1061465}{1}+\frac{792360}{1}+\frac{143860}{1}+\frac{-84240}{1}+\frac{224240}{1}+\frac{0}{1}+\frac{154240}{1}+\frac{0}{1} \\
& \frac{-540220}{1}+\frac{517280}{1}+\frac{141280}{1}+\frac{-27920}{1}+\frac{823120}{1}+\frac{0}{1}+\frac{-22880}{1}+\frac{0}{1} \\
& \frac{240}{1}+\frac{240}{1}+\frac{240}{1}+\frac{240}{1}+\frac{4560}{1}+\frac{0}{1}+\frac{-140}{1}+\frac{0}{1} \\
& \frac{560}{1}+\frac{10560}{1}+\frac{1360}{1}+\frac{-240}{1}+\frac{8240}{1}+\frac{0}{1}+\frac{240}{1}+\frac{0}{1} \\
& \frac{6060}{1}+\frac{-1140}{1}+\frac{-340}{1}+\frac{60}{1}+\frac{-60}{1}+\frac{0}{1}+\frac{-60}{1}+\frac{0}{1} \\
& \frac{-1870}{1}+\frac{330}{1}+\frac{2130}{1}+\frac{-470}{1}+\frac{-330}{1}+\frac{0}{1}+\frac{-330}{1}+\frac{0}{1} \\
& \frac{-240}{1}+\frac{-240}{1}+\frac{-240}{1}+\frac{-240}{1}+\frac{70640}{1}+\frac{0}{1}+\frac{-4560}{1}+\frac{0}{1} \\
& \frac{148080}{1}+\frac{-353520}{1}+\frac{-351120}{1}+\frac{-259920}{1}+\frac{32723920}{1}+\frac{0}{1}+\frac{-2704080}{1}+\frac{0}{1} \\
& \frac{9336}{1}+\frac{-17424}{1}+\frac{-16464}{1}+\frac{-10224}{1}+\frac{1423984}{1}+\frac{0}{1}+\frac{-116976}{1}+\frac{0}{1} \\
& \frac{27330}{1}+\frac{-21120}{1}+\frac{-20520}{1}+\frac{13680}{1}+\frac{304720}{1}+\frac{0}{1}+\frac{-13680}{1}+\frac{0}{1}
\end{aligned}
$$




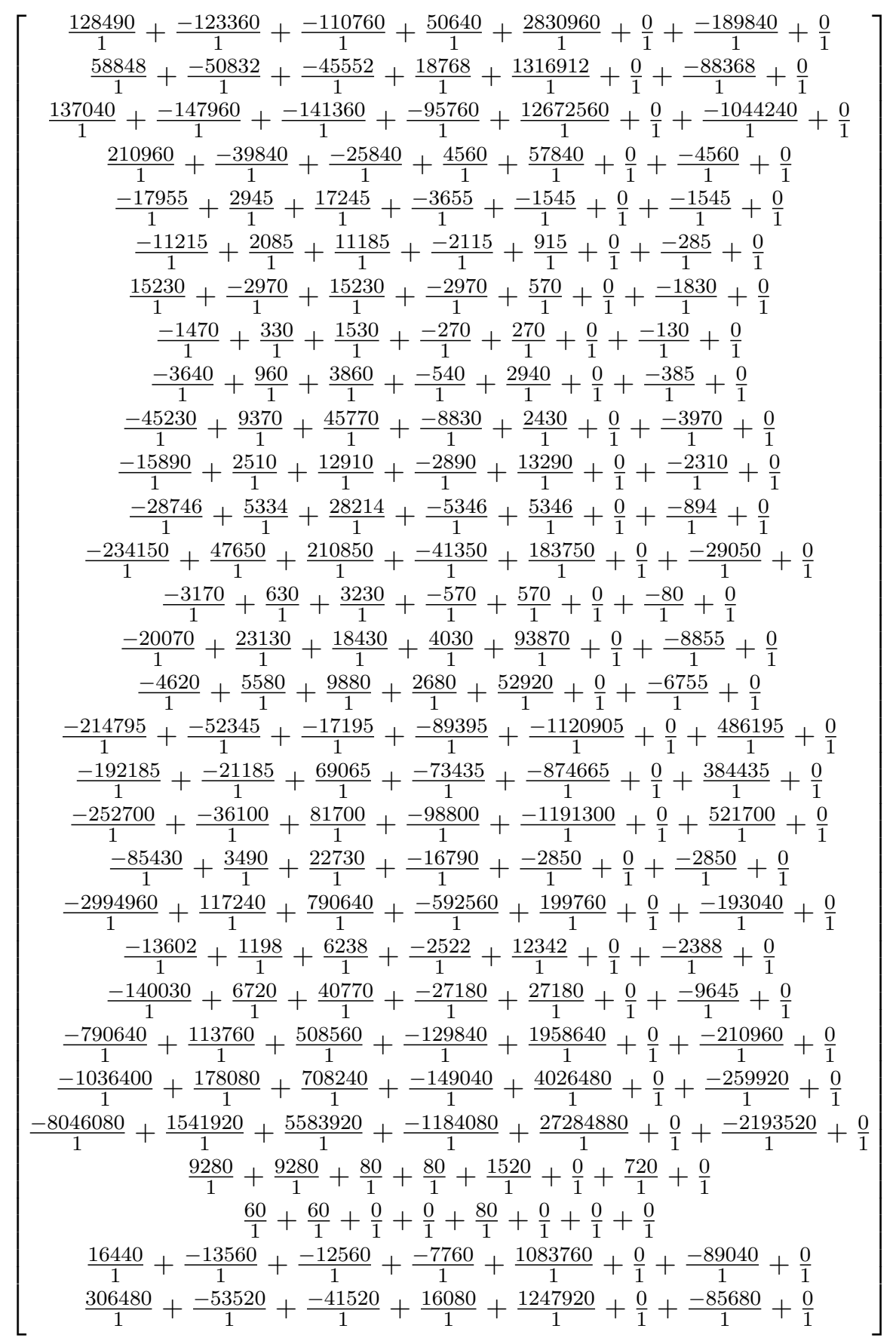




$$
\begin{aligned}
& \frac{125520}{1}+\frac{-16980}{1}+\frac{-13680}{1}+\frac{9120}{1}+\frac{199280}{1}+\frac{0}{1}+\frac{-9120}{1}+\frac{0}{1} \\
& \frac{8840}{1}+\frac{8840}{1}+\frac{40}{1}+\frac{40}{1}+\frac{360}{1}+\frac{0}{1}+\frac{760}{1}+\frac{0}{1} \\
& \frac{705}{1}+\frac{705}{1}+\frac{5}{1}+\frac{5}{1}+\frac{45}{1}+\frac{0}{1}+\frac{45}{1}+\frac{0}{1} \\
& \frac{-880}{1}+\frac{-880}{1}+\frac{-21680}{1}+\frac{-21680}{1}+\frac{-16720}{1}+\frac{0}{1}+\frac{-16720}{1}+\frac{0}{1} \\
& \frac{-13680}{1}+\frac{-13680}{1}+\frac{-28880}{1}+\frac{-28880}{1}+\frac{-259920}{1}+\frac{0}{1}+\frac{7680}{1}+\frac{0}{1} \\
& \frac{417915}{1}+\frac{-78135}{1}+\frac{-52810}{1}+\frac{11040}{1}+\frac{-16890}{1}+\frac{0}{1}+\frac{3585}{1}+\frac{0}{1} \\
& \frac{133920}{1}+\frac{-25080}{1}+\frac{-16480}{1}+\frac{3120}{1}+\frac{-3120}{1}+\frac{0}{1}+\frac{480}{1}+\frac{0}{1} \\
& \frac{6057360}{1}+\frac{-1170240}{1}+\frac{-817840}{1}+\frac{177360}{1}+\frac{-551760}{1}+\frac{0}{1}+\frac{103440}{1}+\frac{0}{1} \\
& \frac{810210}{1}+\frac{-158790}{1}+\frac{-114790}{1}+\frac{26210}{1}+\frac{-110010}{1}+\frac{0}{1}+\frac{24390}{1}+\frac{0}{1} \\
& \frac{23260560}{1}+\frac{-4555440}{1}+\frac{-3413440}{1}+\frac{916560}{1}+\frac{-3105360}{1}+\frac{0}{1}+\frac{725040}{1}+\frac{0}{1} \\
& \frac{4091940}{1}+\frac{-805860}{1}+\frac{-576560}{1}+\frac{127840}{1}+\frac{-515040}{1}+\frac{0}{1}+\frac{93960}{1}+\frac{0}{1} \\
& \frac{17}{1}+\frac{-3}{1}+\frac{17}{1}+\frac{-3}{1}+\frac{-9}{1}+\frac{0}{1}+\frac{3}{1}+\frac{0}{1} \\
& \frac{9248440}{1}+\frac{-1946360}{1}+\frac{-1296560}{1}+\frac{162640}{1}+\frac{-1287440}{1}+\frac{0}{1}+\frac{105760}{1}+\frac{0}{1} \\
& \frac{22877976}{1}+\frac{-5079384}{1}+\frac{-3414224}{1}+\frac{404016}{1}+\frac{-3778416}{1}+\frac{0}{1}+\frac{313344}{1}+\frac{0}{1} \\
& \frac{590260}{1}+\frac{-120740}{1}+\frac{-75240}{1}+\frac{12360}{1}+\frac{-27960}{1}+\frac{0}{1}+\frac{-20160}{1}+\frac{0}{1} \\
& \frac{4321080}{1}+\frac{-886920}{1}+\frac{-548920}{1}+\frac{92280}{1}+\frac{-170280}{1}+\frac{0}{1}+\frac{-170280}{1}+\frac{0}{1} \\
& \frac{1890295}{1}+\frac{-385955}{1}+\frac{-238080}{1}+\frac{39720}{1}+\frac{-59220}{1}+\frac{0}{1}+\frac{-78720}{1}+\frac{0}{1} \\
& \frac{2929040}{1}+\frac{-6601360}{1}+\frac{-6555760}{1}+\frac{-4822960}{1}+\frac{-49182640}{1}+\frac{0}{1}+\frac{1588160}{1}+\frac{0}{1} \\
& \frac{5035}{1}+\frac{-3990}{1}+\frac{-5415}{1}+\frac{3610}{1}+\frac{-3610}{1}+\frac{0}{1}+\frac{3190}{1}+\frac{0}{1} \\
& \frac{71079}{1}+\frac{-60686}{1}+\frac{-72371}{1}+\frac{38114}{1}+\frac{-98914}{1}+\frac{0}{1}+\frac{39726}{1}+\frac{0}{1} \\
& \frac{111188}{1}+\frac{-206492}{1}+\frac{-170012}{1}+\frac{-54492}{1}+\frac{-1381908}{1}+\frac{0}{1}+\frac{572172}{1}+\frac{0}{1} \\
& \frac{1637496}{1}+\frac{-3041064}{1}+\frac{-3127704}{1}+\frac{29336}{1}+\frac{-20351736}{1}+\frac{0}{1}+\frac{8689224}{1}+\frac{0}{1} \\
& \frac{241680}{1}+\frac{-579120}{1}+\frac{-594320}{1}+\frac{-411920}{1}+\frac{-4436880}{1}+\frac{0}{1}+\frac{165120}{1}+\frac{0}{1} \\
& \frac{287280}{1}+\frac{-533520}{1}+\frac{-548720}{1}+\frac{-275120}{1}+\frac{-3570480}{1}+\frac{0}{1}+\frac{174720}{1}+\frac{0}{1} \\
& \frac{246160}{1}+\frac{-541040}{1}+\frac{-533840}{1}+\frac{-385040}{1}+\frac{-3982160}{1}+\frac{0}{1}+\frac{132640}{1}+\frac{0}{1} \\
& \frac{15315216}{1}+\frac{-28442544}{1}+\frac{-27259984}{1}+\frac{-17696144}{1}+\frac{-190346256}{1}+\frac{0}{1}+\frac{6683904}{1}+\frac{0}{1} \\
& \frac{8880}{1}+\frac{7980}{1}+\frac{-120}{1}+\frac{80}{1}+\frac{-80}{1}+\frac{0}{1}+\frac{920}{1}+\frac{0}{1} \\
& \frac{80240}{1}+\frac{55040}{1}+\frac{-3760}{1}+\frac{1840}{1}+\frac{-5840}{1}+\frac{0}{1}+\frac{8160}{1}+\frac{0}{1} \\
& \frac{493160}{1}+\frac{-88540}{1}+\frac{-108240}{1}+\frac{72160}{1}+\frac{-72160}{1}+\frac{0}{1}+\frac{62840}{1}+\frac{0}{1} \\
& \frac{5711680}{1}+\frac{-1029920}{1}+\frac{-1175520}{1}+\frac{693680}{1}+\frac{-1233680}{1}+\frac{0}{1}+\frac{656320}{1}+\frac{0}{1}
\end{aligned}
$$




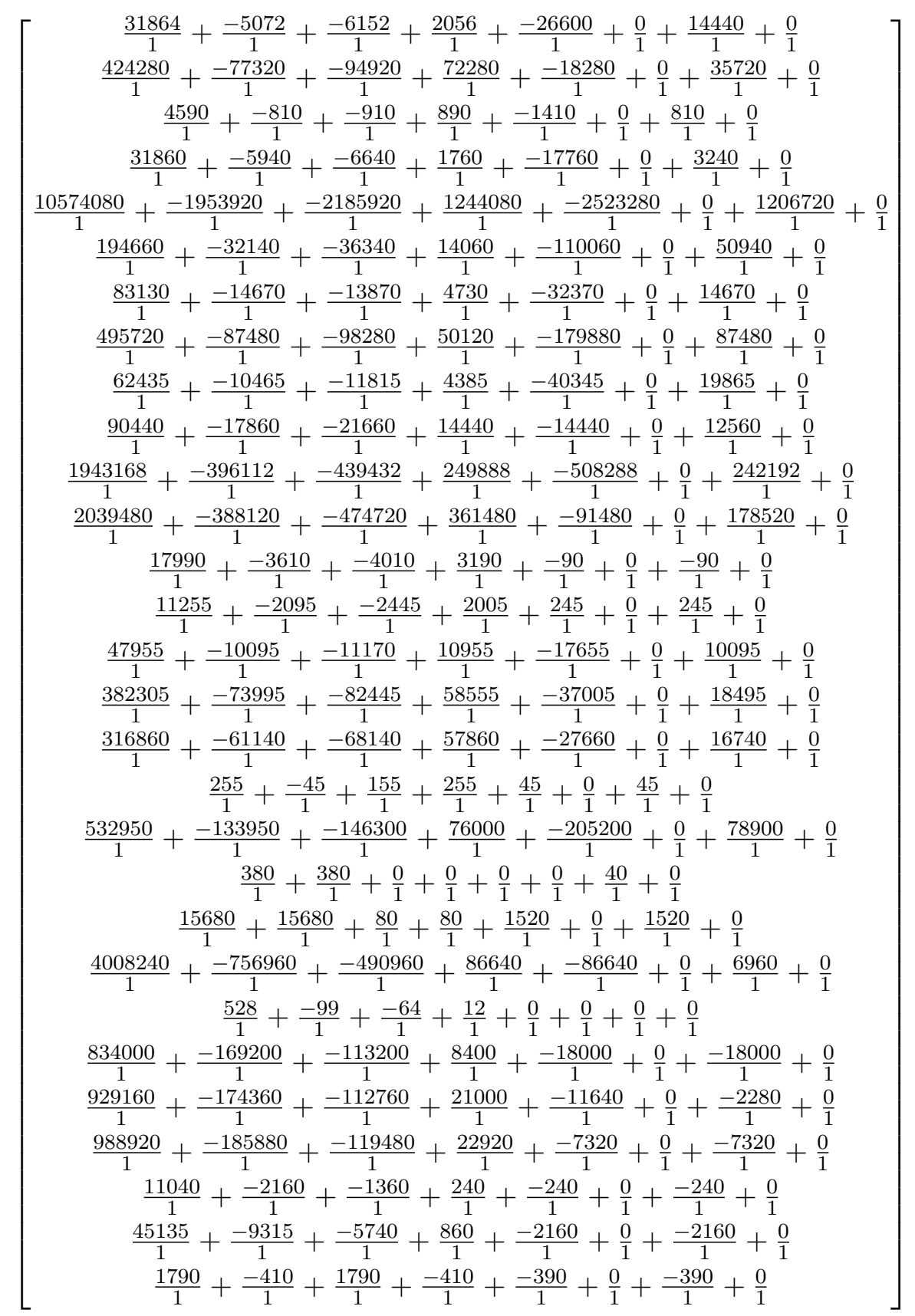

Com as inequações acima, o próximo passo é a programação linear para achar os novos limites do conjunto credal $K(D, C, S)$ :

$$
\left[\begin{array}{c}
\frac{3}{400} \leq D_{1}, C_{1}, S_{1} \leq \frac{3}{50} \\
\frac{9}{400} \leq D_{1}, C_{1}, S_{2} \leq \frac{33}{200} \\
\frac{1}{40} \leq D_{1}, C_{2}, S_{1} \leq \frac{3}{25} \\
\frac{3}{40} \leq D_{1}, C_{2}, S_{2} \leq \frac{33}{100} \\
\frac{3}{1600} \leq D_{2}, C_{1}, S_{1} \leq \frac{1}{40} \\
\frac{19}{800} \leq D_{2}, C_{1}, S_{2} \leq \frac{3}{50} \\
\frac{51}{100} \leq D_{2}, C_{2}, S_{1} \leq \frac{77}{100} \\
\frac{0}{1} \leq D_{2}, C_{2}, S_{2} \leq \frac{0}{1}
\end{array}\right]
$$


Somar vértices $K(C, S)=\sum_{\{D, C, S\} \backslash\{C, S\}} K(D, C, S)$ que resulta na matrix de vértices abaixo:

$\left[\begin{array}{cccc}\frac{13}{400} & \frac{159}{200} & \frac{33}{400} & \frac{9}{100} \\ \frac{469}{50000} & \frac{159}{200} & \frac{33}{400} & \frac{707}{6250} \\ \frac{19}{400} & \frac{159}{200} & \frac{33}{400} & \frac{3}{40} \\ \frac{1547}{25000} & \frac{39031}{50000} & \frac{33}{400} & \frac{3}{40} \\ \frac{1547}{25000} & \frac{159}{200} & \frac{1703}{25000} & \frac{3}{40} \\ \frac{469}{50000} & \frac{14453}{25000} & \frac{33}{400} & \frac{33}{100} \\ \frac{469}{50000} & \frac{159}{200} & \frac{6031}{50000} & \frac{3}{40} \\ \frac{469}{50000} & \frac{34531}{50000} & \frac{9}{40} & \frac{3}{40} \\ \frac{1547}{25000} & \frac{15953}{25000} & \frac{9}{40} & \frac{3}{40} \\ \frac{469}{50000} & \frac{34531}{50000} & \frac{9}{40} & \frac{3}{40} \\ \frac{1547}{25000} & \frac{15953}{25000} & \frac{9}{40} & \frac{3}{40} \\ \frac{469}{50000} & \frac{5207}{6250} & \frac{33}{400} & \frac{3}{40} \\ \frac{469}{50000} & \frac{5207}{6250} & \frac{33}{400} & \frac{3}{40} \\ \frac{1547}{25000} & \frac{39031}{50000} & \frac{33}{400} & \frac{3}{40} \\ \frac{19}{400} & \frac{159}{200} & \frac{33}{400} & \frac{3}{40} \\ \frac{17}{200} & \frac{303}{400} & \frac{33}{400} & \frac{3}{40} \\ \frac{13}{400} & \frac{111}{200} & \frac{33}{400} & \frac{33}{100} \\ \frac{21}{400} & \frac{107}{200} & \frac{33}{400} & \frac{33}{100} \\ \frac{17}{200} & \frac{107}{200} & \frac{33}{400} & \frac{119}{400} \\ \frac{21}{400} & \frac{107}{200} & \frac{33}{400} & \frac{33}{100} \\ \frac{1547}{25000} & \frac{107}{200} & \frac{33}{400} & \frac{16031}{50000} \\ \frac{1547}{25000} & \frac{107}{200} & \frac{457}{6250} & \frac{33}{100} \\ \frac{17}{200} & \frac{107}{200} & \frac{1}{20} & \frac{33}{100} \\ \frac{13}{400} & \frac{107}{200} & \frac{41}{400} & \frac{33}{100} \\ \frac{13}{400} & \frac{107}{200} & \frac{9}{40} & \frac{83}{400} \\ \frac{17}{200} & \frac{107}{200} & \frac{9}{40} & \frac{31}{200} \\ \frac{13}{400} & \frac{63}{100} & \frac{9}{40} & \frac{9}{80} \\ \frac{7}{100} & \frac{63}{100} & \frac{9}{40} & \frac{3}{40} \\ \frac{17}{200} & \frac{123}{200} & \frac{9}{40} & \frac{3}{40} \\ \frac{7}{100} & \frac{63}{100} & \frac{9}{40} & \frac{3}{40}\end{array}\right]$




$\left[\begin{array}{cccc}\frac{17}{200} & \frac{63}{100} & \frac{21}{100} & \frac{3}{40} \\ \frac{469}{50000} & \frac{107}{200} & \frac{6281}{50000} & \frac{33}{100} \\ \frac{469}{50000} & \frac{107}{200} & \frac{9}{40} & \frac{11531}{50000} \\ \frac{1547}{25000} & \frac{107}{200} & \frac{9}{40} & \frac{4453}{25000} \\ \frac{469}{50000} & \frac{63}{100} & \frac{9}{40} & \frac{6781}{50000} \\ \frac{1547}{25000} & \frac{63}{100} & \frac{9}{40} & \frac{1039}{12500} \\ \frac{13}{400} & \frac{111}{200} & \frac{33}{400} & \frac{33}{100} \\ \frac{13}{400} & \frac{63}{100} & \frac{33}{400} & \frac{51}{200} \\ \frac{17}{200} & \frac{63}{100} & \frac{33}{400} & \frac{81}{400} \\ \frac{17}{200} & \frac{63}{100} & \frac{21}{100} & \frac{3}{40} \\ \frac{469}{50000} & \frac{14453}{25000} & \frac{33}{400} & \frac{33}{100} \\ \frac{469}{50000} & \frac{63}{100} & \frac{33}{400} & \frac{6953}{25000} \\ \frac{1547}{25000} & \frac{63}{100} & \frac{33}{400} & \frac{11281}{50000} \\ \frac{13}{400} & \frac{159}{200} & \frac{39}{400} & \frac{3}{40} \\ \frac{13}{400} & \frac{267}{400} & \frac{9}{40} & \frac{3}{40} \\ \frac{17}{200} & \frac{123}{200} & \frac{9}{40} & \frac{3}{40} \\ \frac{13}{400} & \frac{267}{400} & \frac{9}{40} & \frac{3}{40} \\ \frac{13}{400} & \frac{159}{200} & \frac{37}{800} & \frac{101}{800} \\ \frac{469}{50000} & \frac{159}{200} & \frac{37}{800} & \frac{14937}{100000} \\ \frac{1547}{25000} & \frac{159}{200} & \frac{37}{800} & \frac{9687}{100000} \\ \frac{1547}{25000} & \frac{159}{200} & \frac{1703}{25000} & \frac{3}{40} \\ \frac{469}{50000} & \frac{61437}{100000} & \frac{37}{800} & \frac{33}{100} \\ \frac{1547}{25000} & \frac{56187}{100000} & \frac{37}{800} & \frac{33}{100} \\ \frac{469}{50000} & \frac{159}{200} & \frac{6031}{50000} & \frac{3}{40} \\ \frac{469}{50000} & \frac{72687}{100000} & \frac{151}{800} & \frac{3}{40} \\ \frac{1547}{25000} & \frac{67437}{100000} & \frac{151}{800} & \frac{3}{40} \\ \frac{469}{50000} & \frac{72687}{100000} & \frac{151}{800} & \frac{3}{40} \\ \frac{1547}{25000} & \frac{67437}{100000} & \frac{151}{800} & \frac{3}{40} \\ \frac{469}{50000} & \frac{86937}{100000} & \frac{37}{800} & \frac{3}{40} \\ \frac{1547}{25000} & \frac{81687}{100000} & \frac{37}{800} & \frac{3}{40}\end{array}\right]$




\begin{tabular}{|c|c|c|c|}
\hline 469 & 86937 & 37 & 3 \\
\hline$\overline{50000}$ & $\overline{100000}$ & $\overline{800}$ & $\overline{40}$ \\
\hline 1547 & 81687 & 37 & 3 \\
\hline$\overline{25000}$ & $\overline{100000}$ & $\overline{800}$ & $\overline{40}$ \\
\hline 67 & 159 & 37 & 3 \\
\hline$\overline{800}$ & $\overline{200}$ & $\overline{800}$ & $\overline{40}$ \\
\hline 67 & 159 & 37 & 3 \\
\hline$\overline{800}$ & $\overline{200}$ & $\overline{800}$ & $\overline{40}$ \\
\hline 17 & 127 & 37 & 3 \\
\hline$\overline{200}$ & $\overline{160}$ & $\overline{800}$ & $\overline{40}$ \\
\hline 13 & 473 & 37 & 33 \\
\hline$\overline{400}$ & $\overline{800}$ & $\overline{800}$ & $\overline{100}$ \\
\hline 17 & 431 & 37 & 33 \\
\hline$\overline{200}$ & $\overline{800}$ & $\overline{800}$ & $\overline{100}$ \\
\hline 17 & 107 & 1 & 33 \\
\hline 200 & $\overline{200}$ & $\overline{20}$ & $\overline{100}$ \\
\hline 1547 & 107 & 457 & 33 \\
\hline$\overline{25000}$ & $\overline{200}$ & $\overline{6250}$ & $\overline{100}$ \\
\hline 13 & 107 & 41 & 33 \\
\hline$\overline{400}$ & $\overline{200}$ & $\overline{400}$ & $\overline{100}$ \\
\hline 13 & 107 & 151 & 39 \\
\hline$\overline{400}$ & $\overline{200}$ & $\overline{800}$ & $\overline{160}$ \\
\hline 17 & 107 & 151 & 153 \\
\hline$\overline{200}$ & $\overline{200}$ & $\overline{800}$ & $\overline{800}$ \\
\hline 13 & 63 & 151 & 119 \\
\hline$\overline{400}$ & $\overline{100}$ & $\overline{800}$ & $\overline{800}$ \\
\hline 17 & 63 & 151 & 77 \\
\hline$\overline{200}$ & $\overline{100}$ & $\overline{800}$ & $\overline{800}$ \\
\hline 469 & 107 & 6281 & 33 \\
\hline$\overline{50000}$ & $\overline{200}$ & $\overline{50000}$ & $\overline{100}$ \\
\hline 469 & $\underline{107}$ & $\underline{151}$ & 26687 \\
\hline$\overline{50000}$ & $\overline{200}$ & $\overline{800}$ & $\overline{100000}$ \\
\hline 1547 & 107 & 151 & 21437 \\
\hline$\overline{25000}$ & 200 & $\overline{800}$ & $\overline{100000}$ \\
\hline 469 & 63 & 151 & 17187 \\
\hline$\overline{50000}$ & $\overline{100}$ & 800 & 100000 \\
\hline 1547 & 63 & 151 & 11937 \\
\hline$\overline{25000}$ & $\overline{100}$ & $\overline{800}$ & $\overline{100000}$ \\
\hline 13 & $\underline{473}$ & 37 & 33 \\
\hline$\overline{400}$ & $\overline{800}$ & $\overline{800}$ & $\overline{100}$ \\
\hline 13 & 63 & 37 & 233 \\
\hline$\overline{400}$ & $\overline{100}$ & $\overline{800}$ & $\overline{800}$ \\
\hline 17 & 63 & 37 & 191 \\
\hline$\overline{200}$ & $\overline{100}$ & $\overline{800}$ & $\overline{800}$ \\
\hline 469 & 61437 & 37 & 33 \\
\hline$\overline{50000}$ & $\overline{100000}$ & $\overline{800}$ & $\overline{100}$ \\
\hline 469 & 63 & 37 & 31437 \\
\hline$\overline{50000}$ & $\overline{100}$ & $\overline{800}$ & $\overline{100000}$ \\
\hline 1547 & 63 & 37 & 26187 \\
\hline$\overline{25000}$ & $\overline{100}$ & $\overline{800}$ & $\overline{100000}$ \\
\hline 1547 & 56187 & 37 & 33 \\
\hline-25000 & $\overline{100000}$ & $\overline{800}$ & $\overline{100}$ \\
\hline & 431 & 37 & $33]$ \\
\hline & 800 & 800 & $\frac{50}{100}$ \\
\hline & 159 & 39 & 3 \\
\hline & $\overline{200}$ & $\overline{400}$ & $\overline{40}$ \\
\hline & 563 & 151 & 3 \\
\hline & $\overline{800}$ & $\overline{800}$ & $\overline{40}$ \\
\hline & $\underline{521}$ & 151 & 3 \\
\hline & $\overline{800}$ & $\overline{800}$ & $\overline{40}$ \\
\hline & 563 & 151 & 3 \\
\hline & $\overline{800}$ & $\overline{800}$ & $\overline{40}$ \\
\hline & $\underline{521}$ & 151 & 3 \\
\hline & $\overline{800}$ & $\overline{800}$ & $\overline{40}$ \\
\hline & 677 & 37 & 3 \\
\hline & $\overline{800}$ & $\overline{800}$ & $\overline{40}$ \\
\hline & 677 & 37 & 3 \\
\hline & $\overline{800}$ & $\overline{800}$ & $\overline{40}$ \\
\hline & 127 & 37 & 3 \\
\hline & $\overline{160}$ & $\overline{800}$ & $\overline{40}$ \\
\hline & 81 & 33 & 3 \\
\hline & $\overline{100}$ & $\overline{400}$ & $\overline{40}$ \\
\hline & 81 & 33 & 3 \\
\hline & $\overline{100}$ & $\overline{400}$ & $\overline{40}$ \\
\hline & 303 & 33 & 3 \\
\hline & 400 & 400 & 40 \\
\hline
\end{tabular}


Com a matriz de vértices somada, achar o fecho convexo é o próximo passo para então enumerar faces de $K(C, S)$ :

$$
\left[\begin{array}{cccccc}
\frac{-1}{1} & +\frac{1}{1} \cdot K\left(C_{1}, S_{1}\right) & +\frac{1}{1} \cdot K\left(C_{2}, S_{1}\right) & +\frac{1}{1} \cdot K\left(C_{1}, S_{2}\right) & +\frac{1}{1} \cdot K\left(C_{2}, S_{2}\right) \leq 0 \\
\frac{17}{1} & +\frac{-200}{1} \cdot K\left(C_{1}, S_{1}\right) & +\frac{0}{1} \cdot K\left(C_{2}, S_{1}\right) & +\frac{0}{1} \cdot K\left(C_{1}, S_{2}\right) & +\frac{0}{1} \cdot K\left(C_{2}, S_{2}\right) \leq 0 \\
\frac{37}{1} & +\frac{-40}{1} \cdot K\left(C_{1}, S_{1}\right) & +\frac{-40}{1} \cdot K\left(C_{2}, S_{1}\right) & +\frac{-40}{1} \cdot K\left(C_{1}, S_{2}\right) & +\frac{0}{1} \cdot K\left(C_{2}, S_{2}\right) \leq 0 \\
\frac{9}{1} & +\frac{0}{1} \cdot K\left(C_{1}, S_{1}\right) & +\frac{0}{1} \cdot K\left(C_{2}, S_{1}\right) & +\frac{-40}{1} \cdot K\left(C_{1}, S_{2}\right) & +\frac{0}{1} \cdot K\left(C_{2}, S_{2}\right) \leq 0 \\
\frac{-469}{1} & +\frac{50000}{1} \cdot K\left(C_{1}, S_{1}\right) & +\frac{0}{1} \cdot K\left(C_{2}, S_{1}\right) & +\frac{0}{1} \cdot K\left(C_{1}, S_{2}\right) & +\frac{0}{1} \cdot K\left(C_{2}, S_{2}\right) \leq 0 \\
\frac{-67}{1} & +\frac{100}{1} \cdot K\left(C_{1}, S_{1}\right) & +\frac{100}{1} \cdot K\left(C_{2}, S_{1}\right) & +\frac{100}{1} \cdot K\left(C_{1}, S_{2}\right) & +\frac{0}{1} \cdot K\left(C_{2}, S_{2}\right) \leq 0 \\
\frac{-107}{1} & +\frac{0}{1} \cdot K\left(C_{1}, S_{1}\right) & +\frac{200}{1} \cdot K\left(C_{2}, S_{1}\right) & +\frac{0}{1} \cdot K\left(C_{1}, S_{2}\right) & +\frac{0}{1} \cdot K\left(C_{2}, S_{2}\right) \leq 0 \\
\frac{-37}{1} & +\frac{0}{1} \cdot K\left(C_{1}, S_{1}\right) & +\frac{0}{1} \cdot K\left(C_{2}, S_{1}\right) & +\frac{800}{1} \cdot K\left(C_{1}, S_{2}\right) & +\frac{0}{1} \cdot K\left(C_{2}, S_{2}\right) & \leq 0
\end{array}\right]
$$

Com as inequações acima, o próximo passo é a programação linear para achar os novos limites do conjunto credal $K(C, S)$ :

$$
\left[\begin{array}{c}
\frac{469}{50000} \leq C_{1}, S_{1} \leq \frac{17}{200} \\
\frac{37}{800} \leq C_{1}, S_{2} \leq \frac{9}{40} \\
\frac{107}{200} \leq C_{2}, S_{1} \leq \frac{86937}{100000} \\
\frac{3}{40} \leq C_{2}, S_{2} \leq \frac{33}{100}
\end{array}\right]
$$

\section{Regra de Bayes}

Após as eliminações serem executadas, é necessário aplicar a regra de bayes:

$$
K(C \mid S)=\frac{K(C \cap S)}{K(S)}=\frac{\sum_{\{S, D, C, B\} \backslash\{C, S\}}^{\otimes} K(S, D, C, B)}{\sum_{\{S, D, C, B\} \backslash\{S\}}^{\otimes} K(S, D, C, B)}=\frac{K(C, S)}{K(S)}
$$

1. Enumerar vértices de $K(C, S)$

2. Enumerar vértices de $K(S)$

3. Dividir os vértices $K(C, S)$ pelos vértices de $K(S)$, onde $K(C \mid S)=\frac{K(C, S)}{K(S)}$

4. Achar o fecho convexo e então enumerar faces de $K(C \mid S)$

5. Programação Linear $K(C \mid S)$ para achar os novos limites do conjunto credal

Dado os vértices de $K(C, S)$ representados pela matriz a seguir:

$$
\left[\begin{array}{cccc}
\frac{469}{50000} & \frac{107}{200} & \frac{6281}{50000} & \frac{33}{100} \\
\frac{17}{200} & \frac{107}{200} & \frac{1}{20} & \frac{33}{100} \\
\frac{469}{50000} & \frac{61437}{100000} & \frac{37}{800} & \frac{33}{100} \\
\frac{17}{200} & \frac{431}{800} & \frac{37}{800} & \frac{33}{100} \\
\frac{469}{50000} & \frac{107}{200} & \frac{9}{40} & \frac{11531}{50000} \\
\frac{17}{200} & \frac{107}{200} & \frac{9}{40} & \frac{31}{200} \\
\frac{469}{50000} & \frac{34531}{50000} & \frac{9}{40} & \frac{3}{40} \\
\frac{469}{50000} & \frac{86937}{10000} & \frac{37}{800} & \frac{3}{40} \\
\frac{17}{200} & \frac{127}{160} & \frac{37}{800} & \frac{3}{40} \\
\frac{17}{200} & \frac{123}{200} & \frac{9}{40} & \frac{3}{40}
\end{array}\right]
$$

E considerando também os vértices de $K(S)$ representados pela matriz a seguir: 
A divisão dos vértices $K(C, S)$ pelos vértices de $K(S)$, onde $K(C \mid S)=\frac{K(C, S)}{K(S)}$ gerou um conjunto de vértices conforme a matriz abaixo mostra:

$\left[\begin{array}{cccc}\frac{469}{12500} & \frac{107}{50} & \frac{16749}{100000} & \frac{11}{25} \\ \frac{469}{25000} & \frac{107}{100} & \frac{6281}{25000} & \frac{33}{50} \\ \frac{17}{50} & \frac{107}{50} & \frac{6667}{100000} & \frac{11}{25} \\ \frac{17}{100} & \frac{107}{100} & \frac{1}{10} & \frac{33}{50} \\ \frac{469}{12500} & \frac{61437}{25000} & \frac{6167}{100000} & \frac{11}{25} \\ \frac{469}{25000} & \frac{61437}{50000} & \frac{37}{400} & \frac{33}{50} \\ \frac{17}{50} & \frac{431}{200} & \frac{6167}{100000} & \frac{11}{25} \\ \frac{17}{100} & \frac{431}{400} & \frac{37}{400} & \frac{33}{50} \\ \frac{469}{12500} & \frac{107}{50} & \frac{3}{10} & \frac{30749}{100000} \\ \frac{469}{25000} & \frac{107}{100} & \frac{9}{20} & \frac{11531}{25000} \\ \frac{17}{50} & \frac{107}{50} & \frac{3}{10} & \frac{20667}{100000} \\ \frac{17}{100} & \frac{107}{100} & \frac{9}{20} & \frac{31}{100} \\ \frac{469}{12500} & \frac{34531}{12500} & \frac{3}{10} & \frac{1}{10} \\ \frac{469}{25000} & \frac{34531}{25000} & \frac{9}{20} & \frac{3}{20} \\ \frac{469}{12500} & \frac{86937}{25000} & \frac{6167}{100000} & \frac{1}{10} \\ \frac{469}{25000} & \frac{86937}{50000} & \frac{37}{400} & \frac{3}{20} \\ \frac{17}{50} & \frac{127}{40} & \frac{6167}{100000} & \frac{1}{10} \\ \frac{17}{100} & \frac{127}{80} & \frac{37}{400} & \frac{3}{20} \\ \frac{17}{50} & \frac{123}{50} & \frac{3}{10} & \frac{1}{10} \\ \frac{17}{100} & \frac{123}{100} & \frac{9}{20} & \frac{3}{20}\end{array}\right]$

Este conjunto de vértices gerados para $K(C \mid S)$ teve o fecho convexo calculado e as faces enumeradas. As faces geradas do conjunto credal $K(C \mid S)$ podem ser expressos como $b+A x \leq 0$, onde $b$ é um vetor e $A$ é uma matriz de números reais. E $x$ é o vetor dos estados da probabilidade conjunta:

$$
\left[\begin{array}{l}
K\left(C_{1} \mid S_{1}\right) \\
K\left(C_{2} \mid S_{1}\right) \\
K\left(C_{1} \mid S_{2}\right) \\
K\left(C_{2} \mid S_{2}\right)
\end{array}\right]
$$


E a matriz $A$ é representada por:

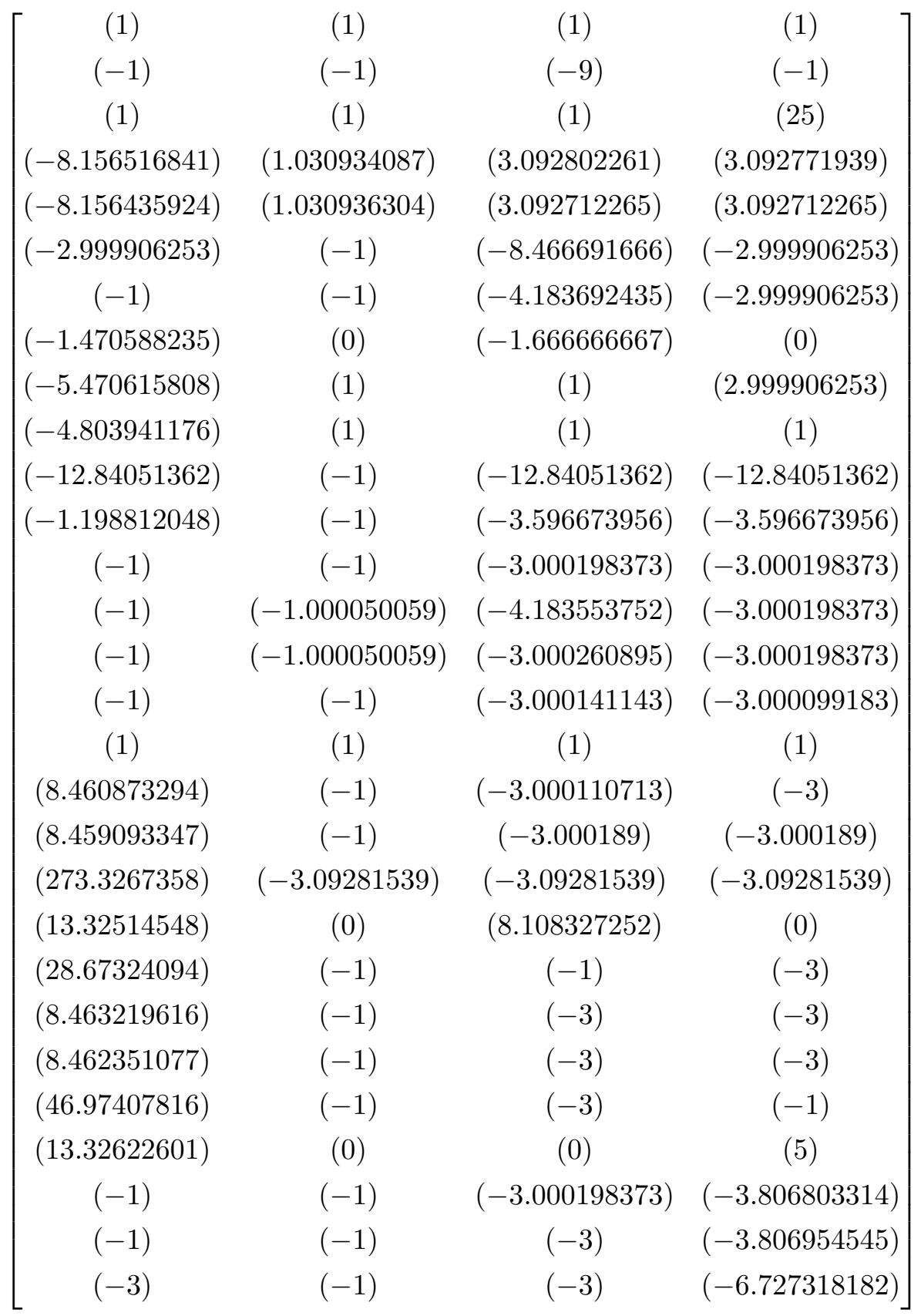


72 INFERÊNCIA EM REDES CREDAIS USANDO TÉCNICAS DE ÁLGEBRA LINEAR (FATORAÇÃO SIMBÓLICA)

$\left[\begin{array}{cccc}(-1.470588235) & (0) & (0) & (-1.136363636) \\ (-4.500058824) & (1) & (3) & (1) \\ (-1) & (-1) & (-1) & (-5.530318182) \\ (1) & (1) & (33.32792734) & (1) \\ (-8.156551832) & (1.030932963) & (3.092842147) & (3.09279889) \\ (-1) & (-1) & (-3) & (-3) \\ (1) & (1) & (52.14077198) & (3) \\ (-1) & (-1) & (-3) & (-2.999906253) \\ (-2.941176471) & (0) & (0) & (0) \\ (1) & (1) & (3) & (33.30017413) \\ (1) & (0) & (0) & (0) \\ (0) & (1) & (0) & (0) \\ (0) & (0) & (1) & (0) \\ (0) & (0) & (0) & (1)\end{array}\right]$

E o vetor real $b$ é:

$\left[\begin{array}{c}(-1) \\ (5.6) \\ (-5.6) \\ (-1) \\ (-1) \\ (6.319966251) \\ (4.355098356) \\ (1) \\ (-1.199981251) \\ (-1.01333) \\ (13.01167767) \\ (4.369922889) \\ (4.000117196) \\ (4.355224249) \\ (4.000236392) \\ (4.000143592) \\ (-2)\end{array}\right]$




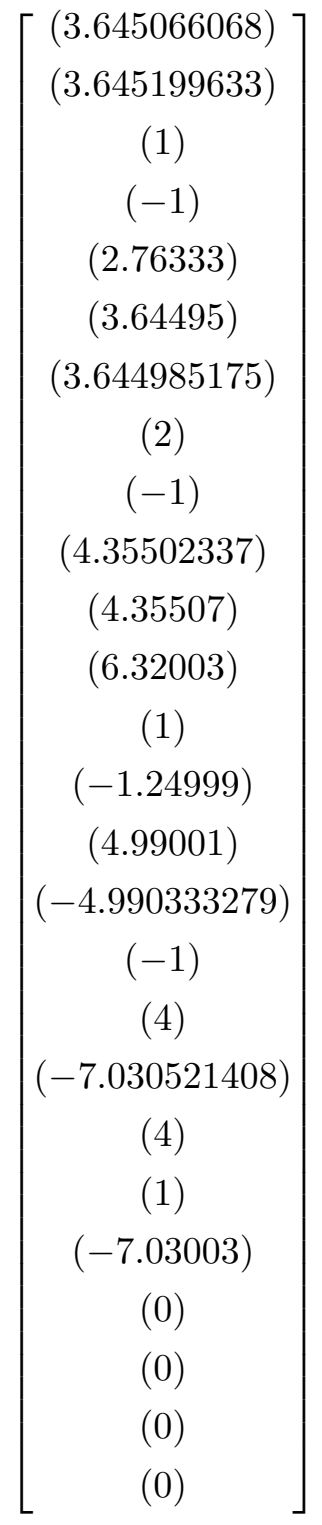

E com programação linear os novos limites do conjunto credal $K(C \mid S)$ encontrados foram:

$$
\left[\begin{array}{rl}
\frac{469}{25000} & \leq C_{1} \mid S_{1} \leq \frac{469}{25000} \\
\frac{6167}{100000} & \leq C_{1} \mid S_{2} \leq \frac{6167}{100000} \\
\frac{107}{100} & \leq C_{2} \mid S_{1} \leq \frac{107}{100} \\
\frac{0}{1} & \leq C_{2} \mid S_{2} \leq \frac{0}{1}
\end{array}\right]
$$


74 INFERÊNCIA EM REDES CREDAIS USANDO TÉCNICAS DE ÁLGEBRA LINEAR (FATORAÇÃO SIMBÓLICA) 


\section{Capítulo 4}

\section{Paralelização da inferência em Redes Credais através de computação distribuída com OpenMPI}

Com a divisão da inferência de redes credais em fatoração simbólica e fatoração numérica, fica claro que a árvore de eliminação é o artefato produzido mais importante para a paralelização. Na árvore de eliminação é estabelcido o balanceamento da carga de trabalho computacional. Enquanto que a fatoração numérica estabelece a funcionalidade de cada unidade computacional disponível para processamento, especificando quais cálculos numéricos devem ser executados.

Este capítulo possui uma pequena introdução no conceito de paralelismo, que será necessário para que o entendimento da implementação da inferência seja pleno. E após esta introdução, o código fonte da biblioteca criada para inferir em redes credais será explicado. Logo depois, os experimentos utilizando a biblioteca de paralelização serão detalhados assim como seus resultados.

\subsection{Computação Paralela}

Com a análise de algoritmos, Cormen et al. (2009), um problema computacional é estudado para ter a sua solução com o algoritmo mais eficiente tanto em questão de recursos de processamento quanto em questão de recursos de armazenamento. Porém, este estudo ao chegar no limite da eficiência de processamento existem outras técnicas para melhorar a capacidade de processamento do algoritmo, chamadas de paralelismo. Sendo este algoritmo implementado por um programa de computador, este programa poderá utilizar essas técnicas de paralelização para diminuir o tempo de processamento e consequentemente o tempo de espera do usuário. O paralelismo é a capacidade de o programa de computador ser executado por mais de uma unidade de processamento, Nagarajan et al. (2013), Grama et al. (2003), Pacheco (1997) e Quinn (2003). Para uma melhor compreensão do paralelismo pode-se utilizar a classificação de como os hardwares dão suporte ao paralelismo chamada taxonomia de Flynn, Flynn (1972), que será introduzida logo a seguir.

\subsubsection{Taxonomia de Flynn}

A Taxonomia de Flynn, Flynn (1972), é uma classificação de como o processamento paralelo trabalhará do ponto de vista de dados e processamento, Nagarajan et al. (2013), Grama et al. (2003) ,Quinn (2003) e Pacheco (1997): 


\begin{tabular}{c|c|c} 
Instruções $\backslash$ Dados & Fluxo único de dados & Fluxo múltiplo de dados \\
\hline Fluxo único de instruções & SISD & SIMD \\
\hline Fluxo múltiplo de instruções & MISD & MIMD
\end{tabular}

Tabela 4.1: Taxonomia de Flynn

Cada entrada na tabela acima 4.1 tem o signficado conforme abaixo:

- SISD - Single Instruction Single Data: Este termo em inglês significa um programa onde existe somente um fluxo de instruções em um fluxo único de dados.

- SIMD - Single Instruction Multiple Data: Este termo em inglês significa um programa onde existe somente um fluxo de instruções em um fluxo de múltiplos dados. Isto significa que uma única instrução será executada de maneira paralela em diferentes fontes de dados.

- MISD - Multiple Instruction Single Data: Este termo em inglês significa um programa onde existe um fluxo de múltipla instruções em um fluxo único de dados. Isto significa que várias e diferentes instruções serão executadas de maneira paralela em uma única e compartilhada fonte de dados.

- MIMD - Multiple Instruction Multiple Data: Este termo em inglês significa um programa onde existe um fluxo de múltipla instruções em um fluxo de múltiplos dados. Isto significa que várias e diferentes instruções serão executadas de maneira paralela em múltiplas fonte de dados. Estas fontes de dados não são necessariamente compartilhadas.

\subsubsection{Computação distribuída - MPI}

Do ponto de vista de dados, o paralelismo pode possuir uma camada de dados compartilhadas ou distribuída. Na camada de dados compartilhada, a memória é vista de maneira igual por todas as unidades de processamento que estão sendo executadas em paralelo, necessitando de mecanismos de controle específicos de paralelismo para leitura e escrita na memória compartilhada. E para a camada de dados distribuída, cada unidade de processamento possui um espaço próprio na memória. E o paralelismo com a camada de dados distribuída possui diversos paradigmas, Tanenbaum e Steen (2002), Grama et al. (2003).

A troca de mensagens é o paradigma de paralelismo de memória distribuída utilizada neste trabalho. MPI - Message Passing Interface é um padrão proposto para uma biblioteca que trabalhe em uma rede de computadores, oferecendo uma interface para passagem de mensagens entre processos independente em qual computador este processo esteja, Pacheco (1997), Quinn (2003) e Grama et al. (2003). OpenMPI é uma biblioteca de código aberto que implementa a MPI para diversos tipos de hardwares, Pacheco (1997).

\subsection{Biblioteca para Inferência em Redes Credais - Organização do código-fonte}

A biblioteca é implementada na linguagem de programação C, Schildt (2001). E o código da biblioteca está organizado em diversas camadas conforme segue abaixo:

- Rede Credal - são as funções para manipulação de arquivos XML no formato BIF, Cozman (2016), que representam redes bayesianas e redes credais, Maranhão (2013). E manipulam também estruturas de dados internas da biblioteca que representam as redes credais.

- Fatoração Simbólica - são as funções que possuem estruturas de dados internas e operações internas da biblioteca para executarem a fatoração simbólica, Colla (2007). 
- Computação distribuída - são as funções que possuem estruturas e operações internas para troca de mensagens específicas de redes credais entre processos de máquinas diferentes utilizando como suporte a biblioteca OpenMPI.

- Envoltória Convexa e Enumeração de vértices de um poliedro - são as funções que executam os cálculos especificados pela fatoração numérica, Avis e Fukuda. (1992), Fukuda e AlainProdon (1996), Fukuda (2016) e Avis (2016).

\subsection{Comparação da inferência linear com a paralela escalável}

\subsubsection{Tempo de execução}

Foram criadas duas redes credais de exemplos conforme anexos que se encontram no fim deste texto. Como a biblioteca de inferência foi desenvolvida para rodar em ambiente linux, a biblioteca utilizada para medição de tempo foi a biblioteca times.h, Bach (1986), Colla (2007).

Foram criadas duas redes credais de exemplos conforme anexos que se encontram no fim deste texto. Como a biblioteca de inferência foi desenvolvida para rodar em ambiente linux, a biblioteca utilizada para medição de tempo foi a biblioteca times.h, Bach (1986), Colla (2007).

O equipamento B utilizado para rodar o experimento da rede credal B do anexo foram duas máquinas virtuais com a configuração :

- Processador: Intel(R) Core(TM) i5-2450M CPU @ 2.50GHz 64 bits

- Memória: $1650 \mathrm{Mb}$

- Rede: 82540EM Gigabit Ethernet Controller - Intel Corporation

- HD: 82801HM/HEM (ICH8M/ICH8M-E) SATA Controller [AHCI mode] - Intel Corporation

- E com o sistema operacional configurado:

- Ubuntu 14.04.1 LTS

- Partição para armazenamento do HD: 7679Mb

- Partição para swap: $510 \mathrm{Mb}$

- Compilador gcc 4.8.4

- Biblioteca OpenMPI libopenmpi1.6.5-8

- Biblioteca C Gnu libc - 2.19

O equipamento A utilizado para rodar o experimento da rede credal A do anexo foi uma máquina virtual com a configuração:

- Processador: Intel(R) Core(TM) i5-2450M CPU @ 2.50GHz 64 bits

- Memória: $2448 \mathrm{Mb}$

- Rede: 82540EM Gigabit Ethernet Controller - Intel Corporation

- HD: 82801HM/HEM (ICH8M/ICH8M-E) SATA Controller [AHCI mode] - Intel Corporation

- E com o sistema operacional configurado:

- Ubuntu 14.10

- Partição para armazenamento do HD: 47Gb

- Sem partição para swap

- Compilador gcc 4.9.1 
- Biblioteca OpenMPI libopenmpi1.6.5-8

- Biblioteca C Gnu libc - 2.19

Com estes equipamentos, os tempos das execuções de ambas redes foram medidos confome a tabela do experimento 4.2 :

\begin{tabular}{c|c|c|c|c} 
Rede Credal & Equipamento & Variáveis Requisitadas & Tempo linear & Tempo distribuído \\
\hline Anexo A & A & 3 variáveis & 552 clock ticks & 654 clock ticks \\
\hline Anexo B & B & 9 variáveis & 12470 clock ticks & 398 clock ticks
\end{tabular}

Tabela 4.2: Experimento de Inferência em Redes Credais

A medida de tempo clock ticks, Bach (1986), é uma medida de ciclo de processamento específica do sistema unix. Existe uma variável de tempo que desde o boot do sistema operacional é atualizada a cada interrupção do relógio, formando a medida de tempo clock ticks. 


\section{Capítulo 5}

\section{Conclusão e trabalhos futuros}

\subsection{Conclusão}

Dado os experimentos, pode-se concluir que o algoritmo de fatoração simbólica ajudou a diminuir o tempo de processamento de inferência em redes credais através do método de eliminação de variáveis. E abriu a oportunidade para que o este método de inferência tire melhor proveito da computação paralela. Em especial dentro da computação paralela, a computação distribuída foi a escolhida para este experimento e traz consigo as propriedades que a inferência irá se beneficiar como :

- Melhora de desempenho: a capacidade de aumentar o poder computacional de maneira que o tempo de processamento da inferência diminua;

- Escalabilidade: que é a capacidade de aumentar a oferta de processamento a medida que a demanda computacional para a inferência cresça;

- Resiliência habilidade de evoluir a solução para construir unidades de processamento que caso não respondam de maneira esperada, sejam substituídas em tempo de processamento sem afetar o processamento da inferência.

E sobre as limitações, a fatoração simbólica utilizada neste trabalho paralelizou somente o método de eliminação de variáveis para inferência aproximadas com o algoritmo $\mathrm{A} / \mathrm{R}+$ em redes credais, da Rocha et al. (2003), logo qualquer outro método de inferência fora a eliminação de variáveis não tem apoio para ser paralelizado.

\subsection{Trabalhos futuros}

O trabalho de associação de fatoração simbólica, Stern (2006) e Stern (1994), a inferência estatística , Colla (2007) e Maranhão (2013), pode ser continuado de diversas formas:

- Outros métodos de inferências aproximadas ou exatas, Antonucci et al. (2014), em redes credais podem ter a fatoração simbólica aplicadas de maneira a paralelizá-los;

- Pode-se estender algumas outras técnicas dentro da fatoração simbólica, Maranhão (2013),Stern (2006) e Stern (1994), para o método de eliminação de variáveis nas redes credais;

- E também a aplicação de fatoração simbólica pode ser aplicado na inferência em outros tipos de redes probabilísticas gráficas, Cozman (2000), Antonucci et al. (2014) e Koller e Friedman (2009). 
80 CONCLUSÃO E TRABALHOS FUTUROS 


\section{Apêndice A}

\section{Rede Credal do exemplo de inferência}

\section{A.1 Rede Credal do exemplo A de inferência}

\section{A.1.1 Rede Credal com limites inferiores}

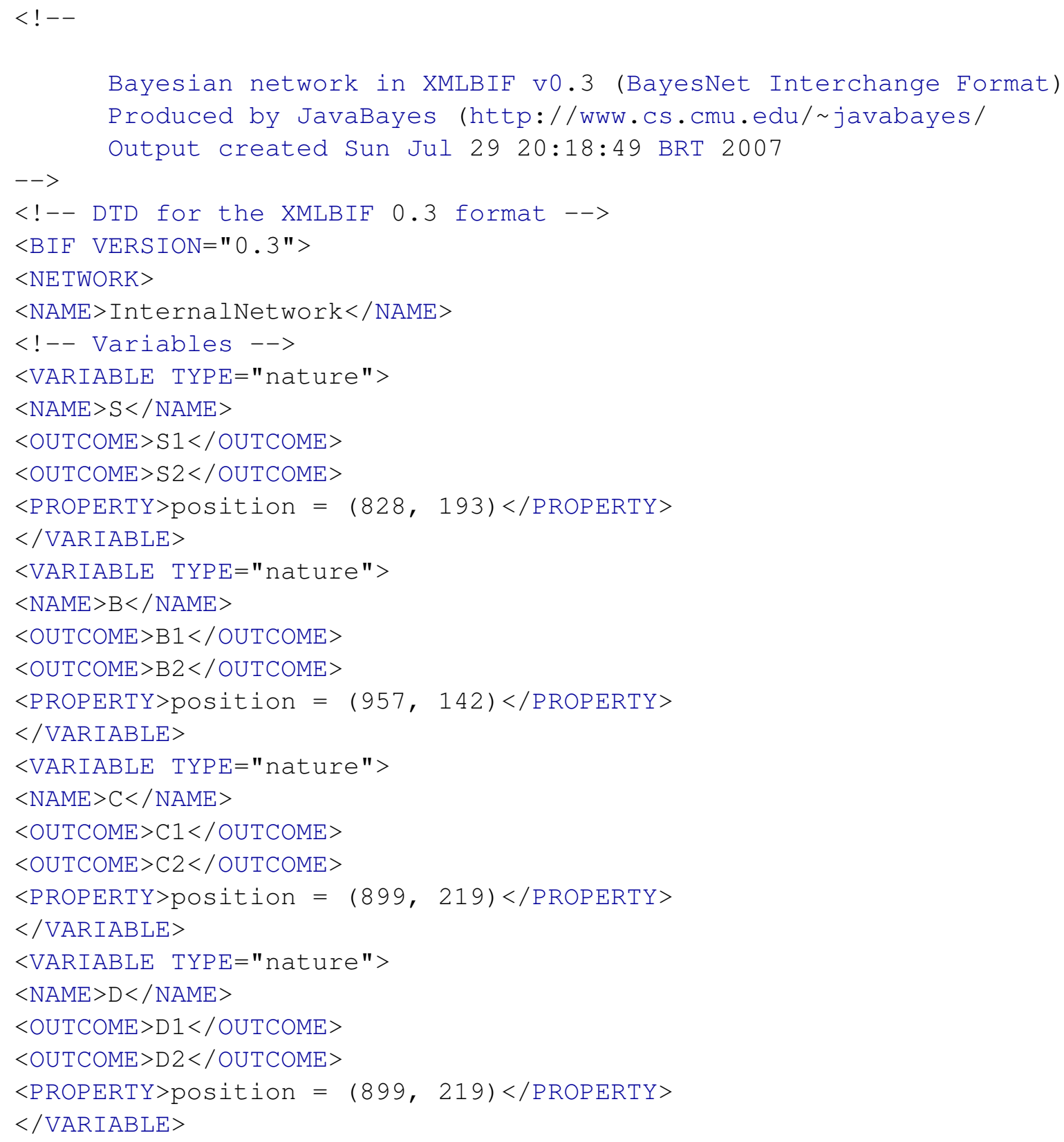




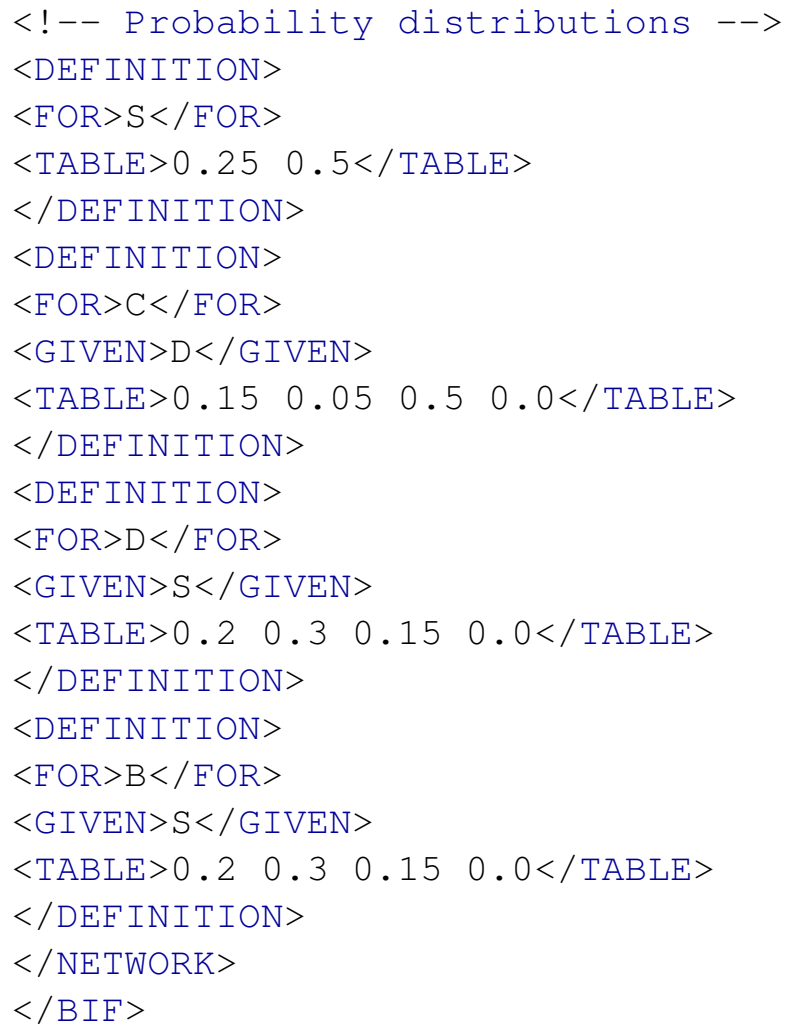




\section{A.1.2 Rede Credal com limites superiores}

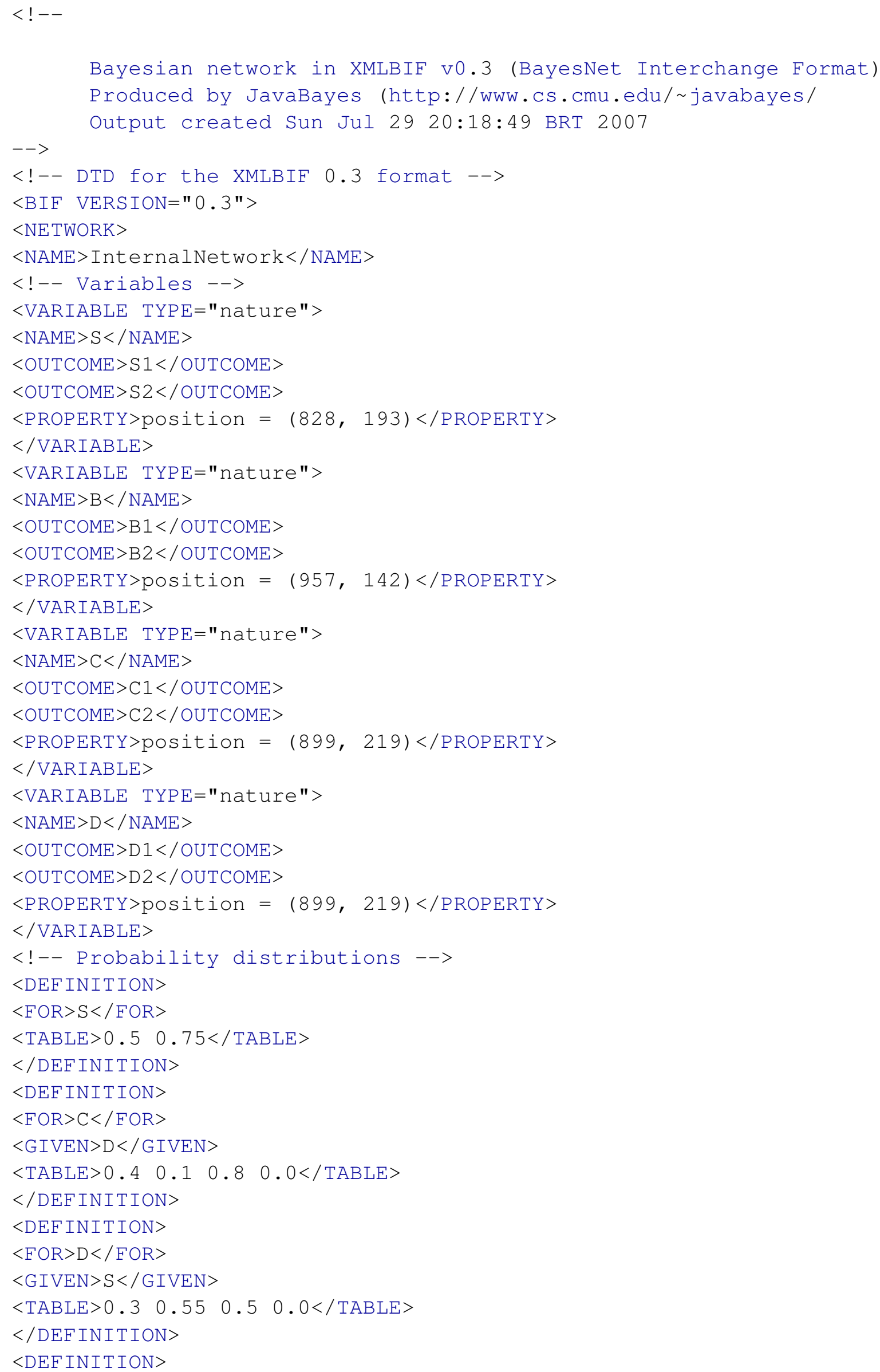


84 APÊNDICE A

$<\mathrm{FOR}>\mathrm{B}</ \mathrm{FOR}>$

$<$ GIVEN $>$ S $</$ GIVEN $>$

$\begin{array}{llll}<\text { TABLE }>0.3 & 0.55 & 0.5 & 0.0</ \text { TABLE }>\end{array}$

$</$ DEFINITION $>$

$</$ NETWORK $>$

$</ \mathrm{BIF}>$ 


\section{A.1.3 Rede Credal Evidência}

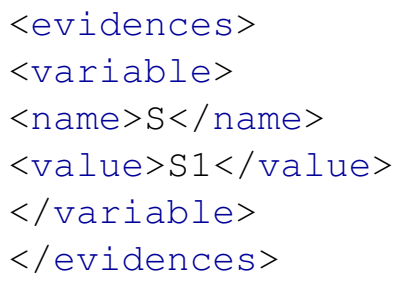




\section{A.1.4 Rede Credal Consulta}

$<$ query $>$

$<$ variable $>$ C $<$ /variable $>$

$</$ query $>$

\section{A.2 Rede Credal do exemplo B de inferência}

\section{A.2.1 Rede Credal com limites inferiores}

$<!--$

Bayesian network in XMLBIF V0.3 (BayesNet Interchange Format)

Produced by JavaBayes (http://www.cs.cmu.edu/ javabayes/

Output created Sun Jul 29 20:18:49 BRT 2007

$-->$

$<$ !-- DTD for the XMLBIF 0.3 format $-->$

$<$ BIF VERSION="0.3">

$<$ NETWORK $>$

$<$ NAME $>$ InternalNetwork $</$ NAME $>$

$<$ !-- Variables - >

$<$ VARIABLE TYPE="nature" $>$

$<\mathrm{NAME}>\mathrm{B}</ \mathrm{NAME}>$

$<O U T C O M E>B 1</ O U T C O M E>$

$<$ OUTCOME $>$ B $2</$ OUTCOME $>$

$<$ PROPERTY $>$ position $=(828,193)</$ PROPERTY $>$

$</$ VARIABLE $>$

$<$ VARIABLE TYPE="nature" $>$

$<\mathrm{NAME}>\mathrm{F}</ \mathrm{NAME}>$

$<$ OUTCOME $>$ F $1</$ OUTCOME $>$

$<O U T C O M E>$ F $2</ O U T C O M E>$

$<$ PROPERTY $>$ position $=(957,142)</$ PROPERTY $>$

$</$ VARIABLE $>$

$<$ VARIABLE TYPE="nature" $>$

$<\mathrm{NAME}>\mathrm{C}</ \mathrm{NAME}>$

$<$ OUTCOME $>$ C $1</$ OUTCOME $>$

$<$ OUTCOME $>$ C $2</$ OUTCOME $>$

$<$ PROPERTY $>$ position $=(899,219)</$ PROPERTY $>$

$</$ VARIABLE $>$

$<$ VARIABLE TYPE="nature" $>$

$<\mathrm{NAME}>\mathrm{D}</ \mathrm{NAME}>$

$<$ OUTCOME $>$ D $1</$ OUTCOME $>$

$<$ OUTCOME $>$ D $2</$ OUTCOME $>$

$<$ PROPERTY $>$ position $=(899,219)</$ PROPERTY $>$

$</$ VARIABLE $>$

$<$ VARIABLE TYPE="nature" $>$

$<\mathrm{NAME}>\mathrm{A}</ \mathrm{NAME}>$

$<O U T C O M E>A 1</ O U T C O M E>$

$<O U T C O M E>A 2</ O U T C O M E>$

$<$ PROPERTY>position $=(899,219)</$ PROPERTY $>$

$</$ VARIABLE $>$

$<$ VARIABLE TYPE="nature" $>$

$<\mathrm{NAME}>\mathrm{E}</ \mathrm{NAME}>$ 


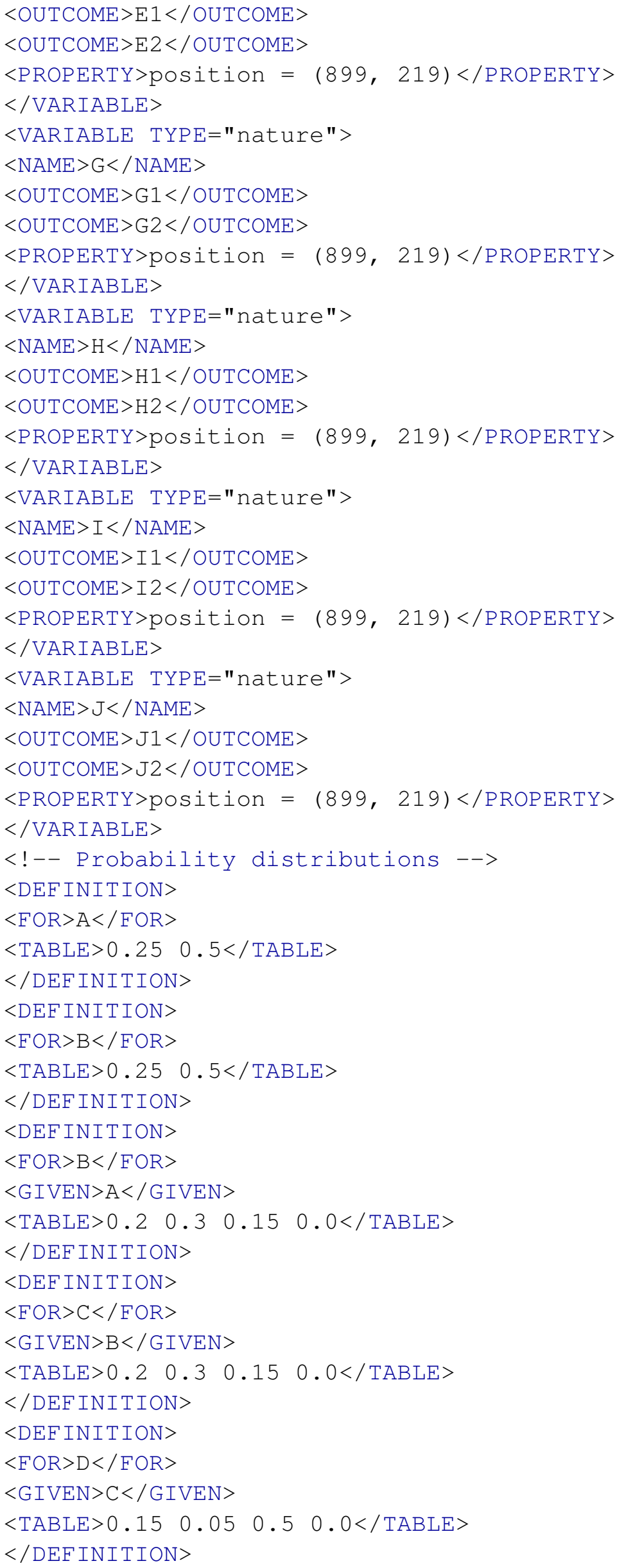




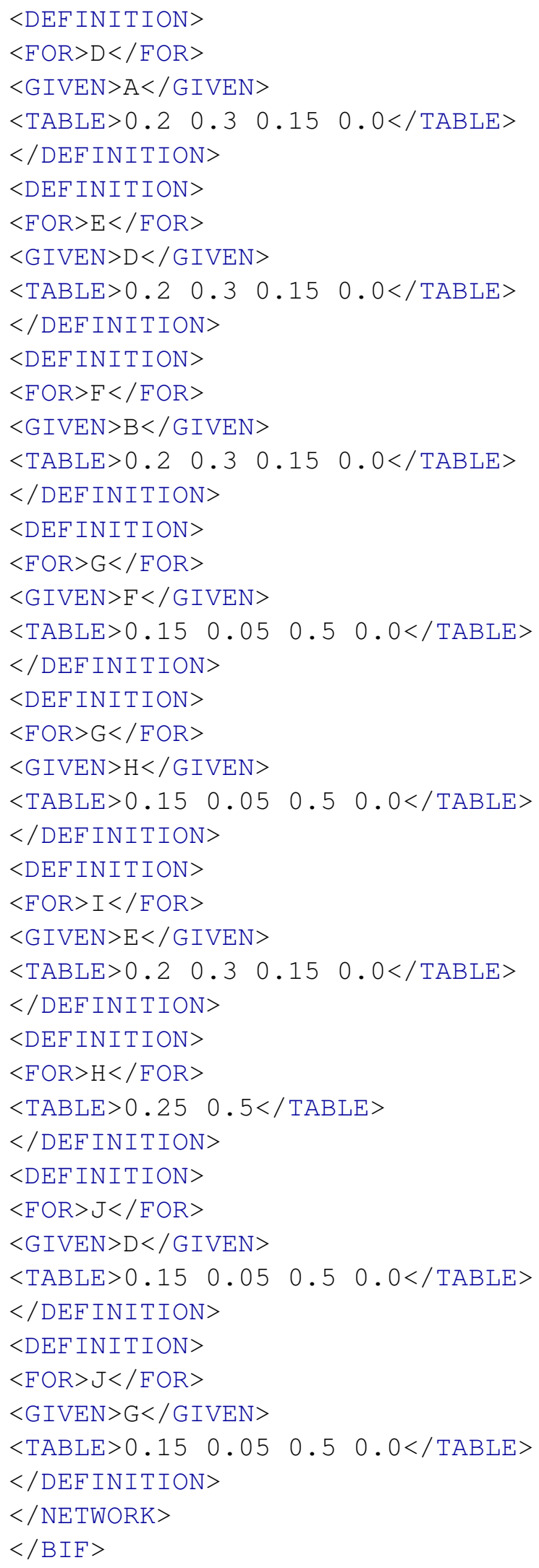




\section{A.2.2 Rede Credal com limites superiores}

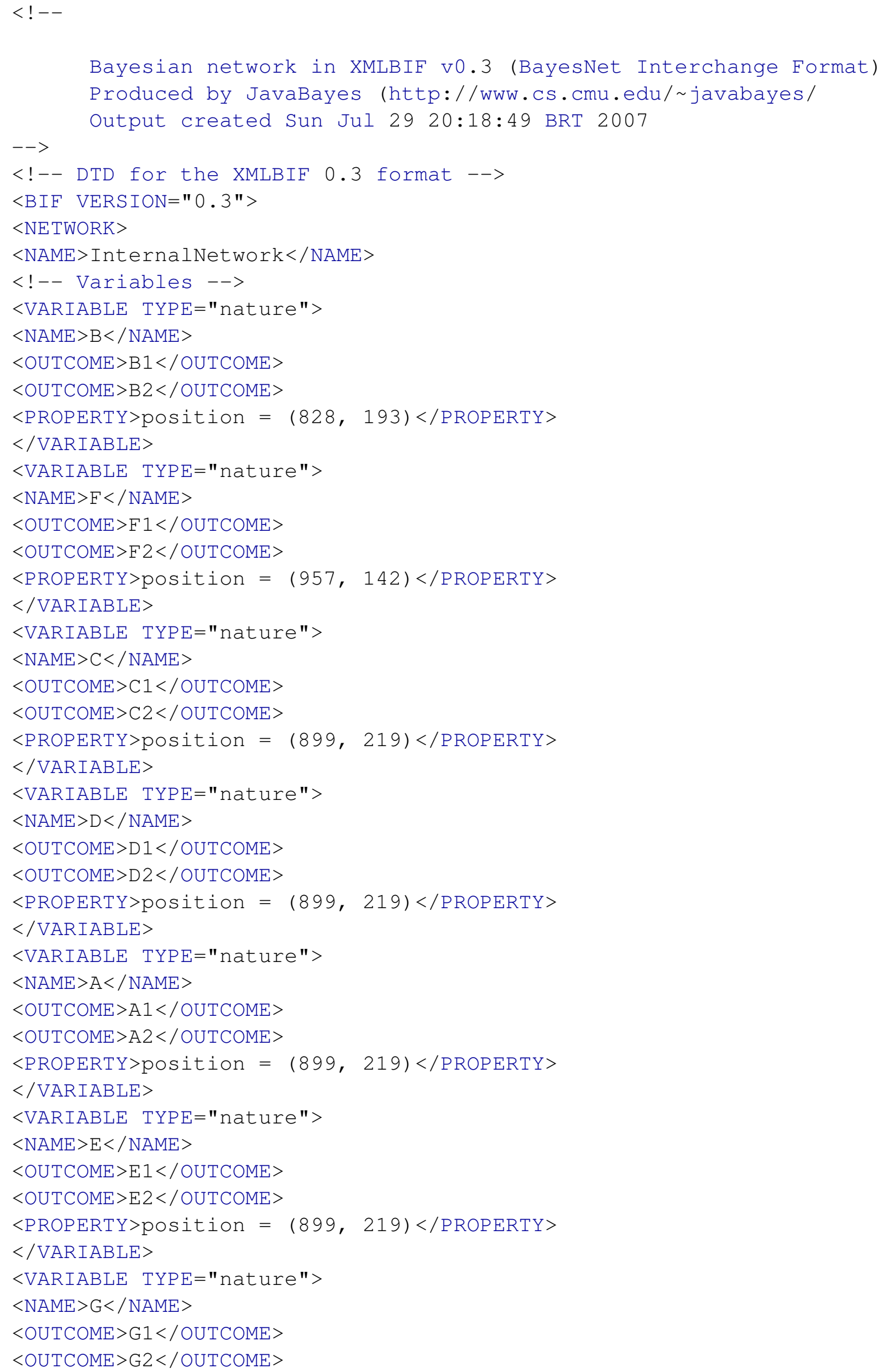




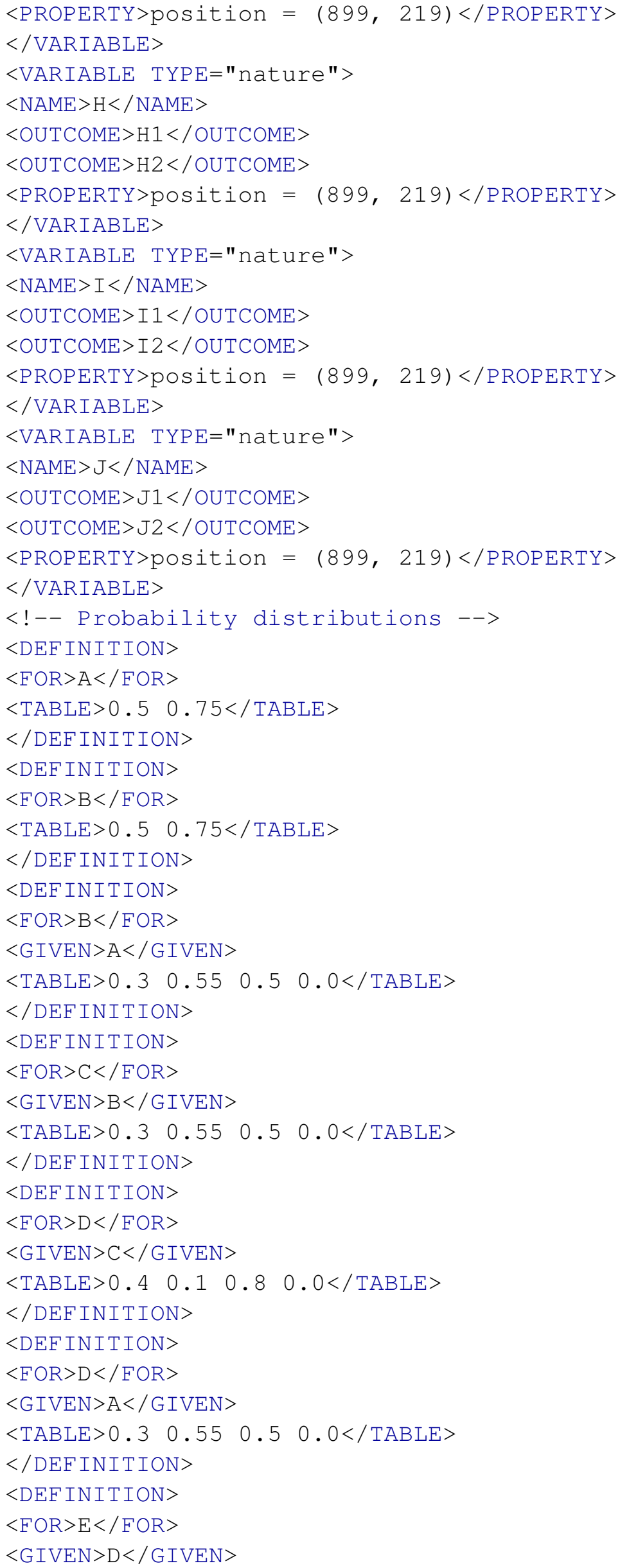




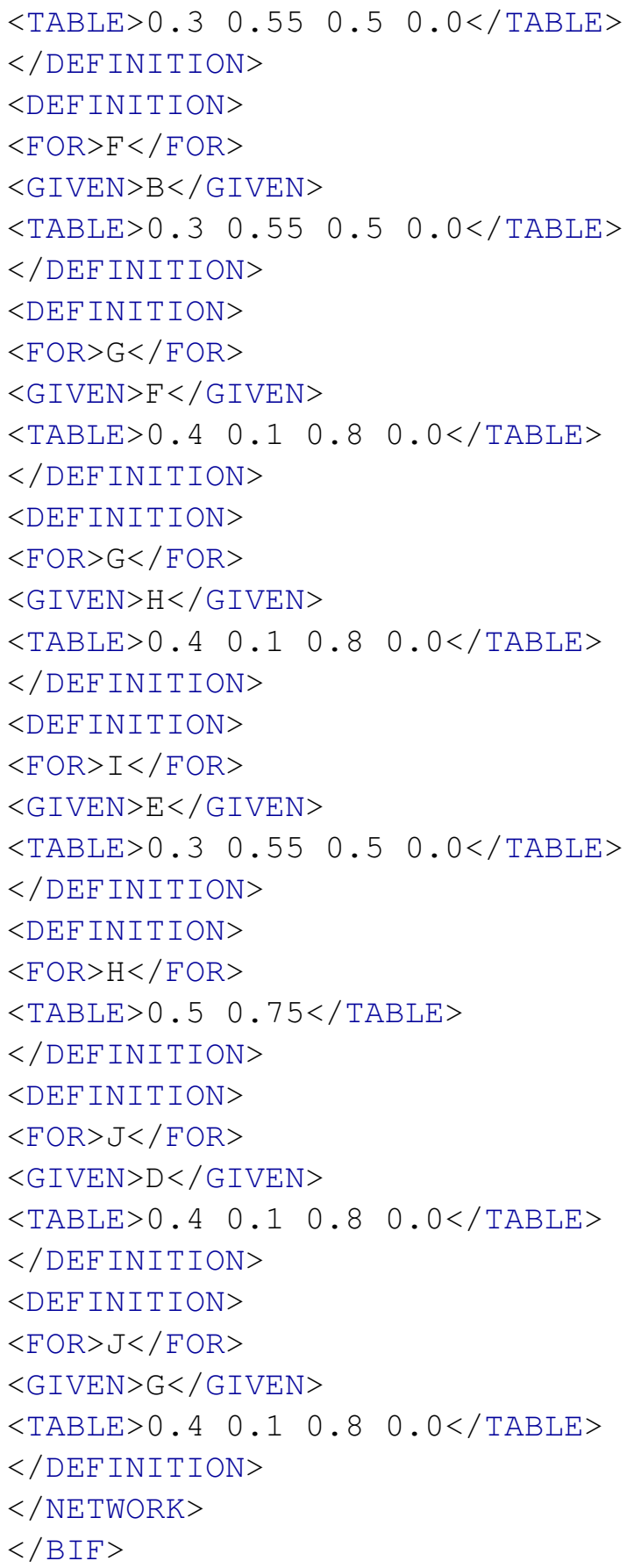




\section{A.2.3 Rede Credal Evidência}

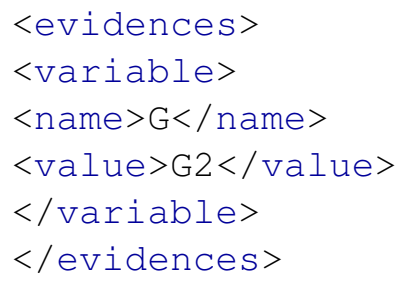




\section{A.2.4 Rede Credal Consulta}

<query>

<variable>I</variable>

$</$ query $>$ 
APÊNDICE A 


\section{Referências Bibliográficas}

Antonucci et al.(2014) A. Antonucci, C. P. de Campos e M. Zaffalon. Introduction to Imprecise Probabilities, chapter Probabilistic Graphical Models. In , Augustin et al. (2014). Citado na pág. 1, $14,15,16,21,36,79$

Augustin et al.(2014) T. Augustin, F. P. A. Coolen, G. Cooman e M. C. M. Troffaes, editors. Introduction to Imprecise Probabilities. Wiley. Citado na pág. 38, 95

Avis e Fukuda.(1992) D. Avis e K. Fukuda. A Pivoting Algorithm for Convex Hulls and Vertex Enumeration of Arrangements and Polyhedra, volume 8. Springer-Verlag, New York. Citado na pág. $15,38,39,77$

Avis(2016) David Avis. lrs home page, Novembro 2016. Disponível no endereço http://cgm.cs. mcgill.ca/ avis/C/lrs.html. Citado na pág. 39, 77

Bach(1986) Maurice J. Bach. The Design of the Unix Operating System. Prentice Hall. ISBN 0-13-201757-1 025. Citado na pág. 77, 78

Bickel e Doksum(2001) P. J. Bickel e K. A. Doksum. Mathematical Statistics Basic Ideas and Selected Topics Volume 1. Prentice Hall, New Jersey. Citado na pág. 12

Bondy e Murty(1982) J. A. Bondy e U. S. R. Murty. Graph Theory with Applications. NorthHolland, Ontario, Canada. Citado na pág. 5, 14

Chartrand e Zhang(2012) G. Chartrand e P. Zhang. A First Course in Graph Theory. Dover, New York, USA. Citado na pág. 4, 5

Colla(2007) Ernesto Coutinho Colla. Aplicação de técnicas de fatoração de matrizes esparsas para inferência em redes bayesianas. Dissertação de Mestrado, Universidade de São Paulo, São Paulo, Brasil. Instituto de Matemática e Estatística. Citado na pág. 13, 17, 18, 19, 21, 22, 25, 29, 30, 32, $36,76,77,79$

Cormen et al.(2009) T. H. Cormen, C. E. Leiserson, R. L. Rivest e C. Stein. Introduction to Algorithms. MIT Press, Third edition. Citado na pág. 1, 15, 38, 75

Costa(1933) N. C. A. Costa. Lógica Indutiva e Probabilidade. HUCITEC: EDUSP - São Paulo. Citado na pág. 6

Cozman(2000) F.G. Cozman. Credal networks. Artificial Intelligence, 120:199-233. Citado na pág. $14,15,79$

Cozman(2016) F.G. Cozman. Formato de XML para Rede Bayesiana, Novembro 2016. Disponível no endereço http://www.cs.cmu.edu/ fgcozman/Research/InterchangeFormat/. Citado na pág. 18, 76

da Rocha et al.(2003) Jose Carlos Ferreira da Rocha, Fabio Gagliardi Cozmanl e Cassio Polpo de Campos. Inference in Polytrees with Sets of Probabilities. UAI'03. Morgan Kaufmann Publishers Inc., San Francisco, CA, USA. ISBN 0-127-05664-5. URL http://dl.acm.org/citation. $\mathrm{cfm} ? \mathrm{id}=2100584.2100610$. Citado na pág. $1,38,79$ 
Degroot e Schervish(2012) M. H. Degroot e M. J. Schervish. Probability and Statistics. Fourth Edition, Pearson Education. Citado na pág. 5, 12

DeGroot(1989) M.H. DeGroot. Probability and Statistics. Addison-Wesley. Citado na pág. 5

Diestel(2000) R. Diestel. Graph Theory. Springer-Verlag, New York, USA. Citado na pág. 3, 4

Flynn(1972) Michael J. Flynn. Some Computer Organizations and Their Effectiveness, volume 21. IEEE Computer Society, Washington, DC, USA. doi: 10.1109/TC.1972.5009071. URL http: //dx.doi.org/10.1109/TC.1972.5009071. Citado na pág. 75

Fukuda(2016) Komei Fukuda. cdd home, Novembro 2016. Disponível no endereço https://www. inf.ethz.ch/personal/fukudak/cdd_home. Citado na pág. 39, 77

Fukuda e AlainProdon(1996) Komei Fukuda e AlainProdon. Double Description Method Revisited. Springer-Verlag, London, UK, UK. ISBN 3-540-61576-8. URL http://dl.acm.org/citation. cfm?id=646124.680737. Citado na pág. 39,77

Grama et al.(2003) Ananth Grama, Anshul Gupta, George Karypis e Vipin Kumar. Introduction to Parallel Computing. Addison Wesley. ISBN 0-201-64865-2. Citado na pág. 75, 76

James(2013) B.R. James. Probabilidade: Um Curso em Nível Intermediário. 3 edição, Rio de Janeiro, IMPA. Citado na pág. 6, 7, 8, 9, 11, 13, 16

Koller e Friedman(2009) D. Koller e N. Friedman. Probabilistic Graphical Models Principles and Techniques. MIT-Press. Citado na pág. 1, 5, 8, 11, 13, 21, 35, 79

Kolmogorov(1950) A. N. Kolmogorov. Foundations of the Theory of Probability. Chelsea, N. York. Tradução para o ingles de Grundbeguiffe der Wahrscheinlichkeitsrechnung(1033). Citado na pág. 6

Maranhão(2013) Viviane Teles Lucca Maranhão. Estudo de técnicas de paralelização de métodos computacionais de fatoração de matrizes esparsas aplicados à redes bayesianas e credais. Dissertação de Mestrado, Universidade de São Paulo, São Paulo, Brasil. Instituto de Matemática e Estatística. Citado na pág. 1, 14, 15, 76, 79

Monticeli(2010) André Rodrigues Monticeli. Um estudo sobre sistemas de inequações lineares. Dissertação de Mestrado, Universidade Estadual de Campinas, Campinas, São Paulo, Brasil. Instituto de Matemática, Estatística e Computação Científica - IMECC. Citado na pág. 38, 39

Nagarajan et al.(2013) Radhakrishnan Nagarajan, Marco Scutari e Sophie Lèbre. Bayesian Networks in R. Springer-Verlag New York. ISBN 978-1-4614-6445-7. Citado na pág. 75

Pacheco(1997) P. S. Pacheco. Parallel Programming with MPI. Morgan Kaufmann. Citado na pág. 75,76

Pearl(1988) J. Pearl. Probabilistic Reasoning in Intelligent Systems: Networks of Plausive Inference. Morgan Kaufmann, San Francisco. Citado na pág. 12

Pissanetzky(2005) S. Pissanetzky. Sparse Matrix Technology. Academic Press, New York, USA. Citado na pág. 29

Pourret e Naim(2008) Olvieir Pourret e Patrick Naim. Bayesian Networks A Pratical Guide to Applications. Wile. Citado na pág. 1

Quinn(2003) Michael J. Quinn. Parallel Programming in C with MPI and OpenMP. McGrawHill, Oregon State University-USA. Citado na pág. 75,76 
Roussas(1997) G. G. Roussas. A Course in Mathematical Statistics. Academic Press, USA. Citado na pág. $6,7,8,9,11,12,16$

Roussas(2003) G. G. Roussas. Introduction to Probability and Statistical Inference. Academic Press, USA. Citado na pág. 6, 7, 8, 9, 11, 12, 16

Schildt(2001) Herbert Schildt. C the Complete Reference. McGraw-Hill Osborne Media. Citado na pág. 76

Stern(1994) J. M. Stern. Esparsidade, Estrutura, Estabilidade e Escalonamento em Álgebra Linear Computacional. IX Escola de Computação (Recife) (Silvio Lemos Meira, ed.),UFPE. Citado na pág. 29,79

Stern(2006) J. M. Stern. Decoupling, Sparsity, Randomization and Objective Bayesian Inference. Relatório Técnico MAC- 2006-07. Citado na pág. 29, 79

Tanenbaum e Steen(2002) Andrew S. Tanenbaum e Maarten Van Steen. Distributed Systems Principles and Paradigms. Prentice Hall, New Jersey. Citado na pág. 76

Tessem(1992) B. Tessem. Interval Probability Propagation, volume 7. Elsevier. Citado na pág. 1 